\title{
Chlamydia and gonorrhea epidemiology
}

Citation for published version (APA):

Wijers, J. (2020). Chlamydia and gonorrhea epidemiology: test practices \& bacterial load. [Doctoral Thesis, Maastricht University]. Boekenplan. https://doi.org/10.26481/dis.20200918jw

Document status and date:

Published: 01/01/2020

DOI:

10.26481/dis.20200918jw

Document Version:

Publisher's PDF, also known as Version of record

\section{Please check the document version of this publication:}

- A submitted manuscript is the version of the article upon submission and before peer-review. There can be important differences between the submitted version and the official published version of record.

People interested in the research are advised to contact the author for the final version of the publication, or visit the DOI to the publisher's website.

- The final author version and the galley proof are versions of the publication after peer review.

- The final published version features the final layout of the paper including the volume, issue and page numbers.

Link to publication

\footnotetext{
General rights rights.

- You may freely distribute the URL identifying the publication in the public portal. please follow below link for the End User Agreement:

www.umlib.nl/taverne-license

Take down policy

If you believe that this document breaches copyright please contact us at:

repository@maastrichtuniversity.nl

providing details and we will investigate your claim.
}

Copyright and moral rights for the publications made accessible in the public portal are retained by the authors and/or other copyright owners and it is a condition of accessing publications that users recognise and abide by the legal requirements associated with these

- Users may download and print one copy of any publication from the public portal for the purpose of private study or research.

- You may not further distribute the material or use it for any profit-making activity or commercial gain

If the publication is distributed under the terms of Article $25 \mathrm{fa}$ of the Dutch Copyright Act, indicated by the "Taverne" license above, 
Chlamydia and Gonorrhea Epidemiology:

Test practices \& Bacterial load

Juliën Wijers 


\section{(c) Copyright: Juliën Wijers}

Cover design: Margret Reijnders | Creatieve Communicatie

Layout: Boekenplan

Printed by: Boekenplan, Maastricht

ISBN 9789086665105

No part of thesis may be reproduced, stored, or transmitted in any form or by any means, electronic or mechanical, including photocopy, recording or any information storage or retrieval system, without permission of the copyright owner.

This thesis was prepared at the Care and Public Health Research Institute (CAPHRI) of Maastricht University, in collaboration with the Department of Medical Microbiology of the Maastricht University Medical Center (MUMC+) and the Department of Sexual Health, Infectious Diseases and Environmental Health at the Public Health Service South Limburg (GGD Zuid-Limburg).

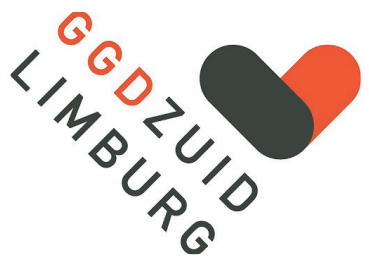




\title{
Chlamydia and Gonorrhea Epidemiology: Test practices \& Bacterial load
}

\author{
PROEFSCHRIFT \\ ter verkrijging van de graad van doctor aan de Universiteit Maastricht, \\ op gezag van de Rector Magnificus, Prof. dr. Rianne M. Letschert \\ volgens het besluit van het college van Decanen, \\ In het openbaar te verdedigen \\ op vrijdag 18 september om 14:00 uur
}

Door

Juliën-Noël Arnold Petronella Wijers

Geboren op 25 december 1991 te Well (LB) 


\section{Promotor:}

Prof. dr. Christian J.P.A. Hoebe

\section{Co-promotors:}

Dr. Geneviève van Liere

Dr. Nicole H.T.M. Dukers-Muijrers

\section{Beoordelingscommissie:}

Prof. dr. Hans Bosma (voorzitter)

Prof. dr. Servaas Morré

Prof. dr. Maria Prins, Amsterdam UMC

Prof. dr. Pim Assendelft, Radboud UMC Nijmegen 


\section{Contents}

Chapter 1 General Introduction

\section{Section retesting}

Chapter 2 Test of cure, retesting and extragenital testing practices for

Chlamydia trachomatis and Neisseria gonorrhoeae among general practitioners in different socioeconomic status areas:

a retrospective cohort study, 2011-2016

Juliën NAP Wijers, Geneviève AFS van Liere, Christian JPA Hoebe, Jochen WL Cals, Petra FG Wolffs, Nicole HTM Dukers-Muijrers PLOS ONE. 2018; 13(3):e019435.

Chapter 3 The characteristics of patients frequently tested and repeatedly

infected with Chlamydia trachomatis in Southwest Limburg, the Netherlands

Juliën N.A.P. Wijers, Nicole H.T.M. Dukers-Muijrers, Christian J.P.A. Hoebe, Petra F.G. Wolffs, Geneviève A.F.S. van Liere BMC Public Health 20, 1239 (2020).

Chapter 4 The characteristics of patients frequently tested and repeatedly infected with Neisseria gonorrhoeae Juliën Wijers, Christian Hoebe, Nicole Dukers-Muijrers, Petra Wolffs, Geneviève van Liere Int. J. Environ. Res. Public Health 2020, 17, 1495.

\section{Section bacterial load}

Chapter 5 Chlamydia trachomatis bacterial load, estimated by Cq values, in urogenital samples from men and women visiting the general practice, hospital or STI clinic Juliën NAP Wijers, Christian JPA Hoebe, Geneviève AFS van Liere, Petra FG Wolffs, Nicole HTM Dukers-Muijrers PLOS ONE 2019; 14(4): e0215606.

Chapter 6 Men and women have an equal oropharyngeal and anorectal

Chlamydia trachomatis bacterial load: a comparison of three anatomic sites Juliën NAP Wijers, Nicole HTM Dukers-Muijrers, Geneviève AFS van Liere, 
Jeanne AMC Dirks, Petra FG Wolffs, Christian JPA Hoebe

The Journal of Infectious Diseases 2019 December 16;

DOI: 10.1093/infdis/jiz668.

Chapter 7 Men and women repeatedly infected with Chlamydia trachomatis

129

have a lower urogenital bacterial load

Juliën N.A.P. Wijers, Geneviève A.F.S. van Liere,

Nicole H.T.M. Dukers-Muijrers, Petra F.G. Wolffs, Christian J.P.A. Hoebe

Sex Transm Dis 2020 doi: 10.1097/OLQ.0000000000001219.

\section{Section geographical analyses}

Chapter 8 The added value of geographical cluster analyses to standard

epidemiological analyses for Chlamydia trachomatis and

Neisseria gonorrhoeae control using Dutch national surveillance data Juliën NAP Wijers, Christian JPA Hoebe, Nicole HTM Dukers-Muijrers, Boris Kauhl, Birgit H van Benthem, Hannelore M Götz, Thomas Krafft, Jürgen Schweikart, Geneviève AFS van Liere

submitted.

Chapter 9 General discussion

Chapter 10 Summary

Nederlandse samenvatting

Valorization

Dankwoord

180

List of publications 


"Combining a PhD with renovating your house makes you Dr. House." 


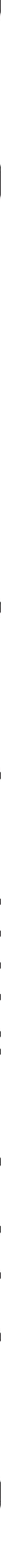




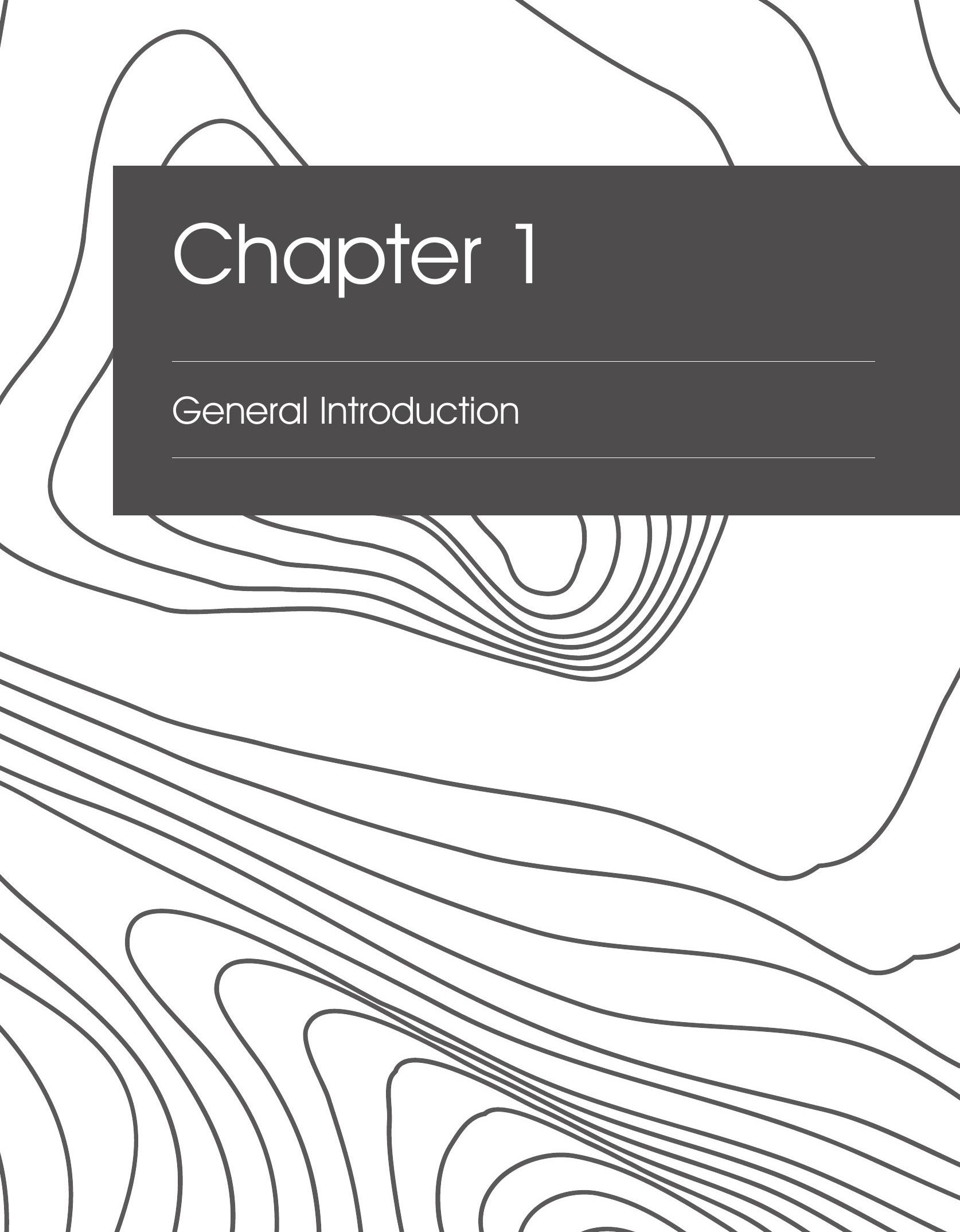




\section{Background}

Chlamydia trachomatis (CT) and Neisseria gonorrhoeae (NG) are the most prevalent bacterial sexually transmitted infections (STIS) worldwide. ${ }^{1}$ CT and NG accounted for 209 million new infections in the year 2012, an average of more than a half million infections each day. ${ }^{1}$ CT and NG are associated with reproductive sequelae such as pelvic inflammatory disease (PID), ectopic pregnancy, infertility and chronic abdominal pain in women, and urethritis and epididymitis in men. ${ }^{2-4}$ Both CT and NG can facilitate the transmission of other STIs such as Human Immunodefiency Virus (HIV). Whereas NG is becoming an emerging global threat due to multidrug resistance. ${ }^{4}$

\section{Chlamydia trachomatis}

\section{Epidemiology of Chlamydia trachomatis in the Netherlands}

CT is the most common reported bacterial STI in the Netherlands. Two main STI care providers test for CT, that are general practitioners (GPS) and STI clinics. GPs perform the majority of STI consultations and diagnoses. ${ }^{5}$

An estimated 39,800 CT infections were diagnosed by GPs in the year 2017. ${ }^{5}$ The number of CT diagnoses increased with $8 \%$ in both men and women in comparison with the year 2016.

The Netherlands counts eight regional Centers for Sexual Health with 24 outpatient STI clinics. Individuals at high risk for STIs are offered free testing at STI clinics. Those are men who have sex with men (MSM), individuals, or partners, originating from STI endemic countries (Suriname, Netherlands Antilles, Turkey, Morocco, Africa, South-America, Asia or Eastern-Europe), individuals younger than 25 years of age, individuals with STI-related symptoms, individuals notified for STIs, victims of rape or sexual violence and commercial sex workers. ${ }^{67}$ According to recent numbers of The Netherlands National Institute for Public Health and the Environment (in Dutch: RIVM), 21,021 CT infections were diagnosed in $2018 .^{5}$ This means $1.8 \%$ less than in the year 2017, while the total number of STI tests increased with $1 \%(n=1,624)$ in $2018 .{ }^{5}$ The CT test positivity rate has increased from the year 2009 to 2016, but has stabilized in the past two years. The CT test positivity rate of women is $15.0 \%$, and for heterosexual men $17.7 \% .{ }^{5}$ Among MSM, the CT test positivity rate is $9.8 \%$ in 2018 and is stable for years (Figure 1). ${ }^{5}$ 


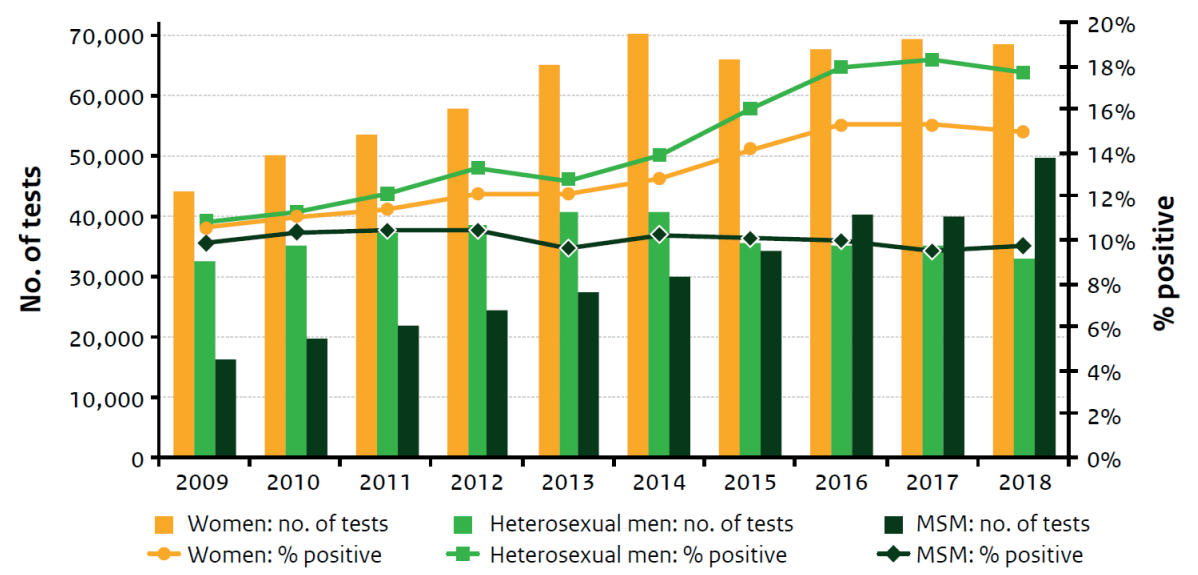

Figure 1. Total number of tests and positivity rate of CT by gender and type of sexual contact, 2009-2018 (Source: Slurink I, van Aar F, Op de Coul E, et al. Sexually transmitted infections in the Netherlands in 2018: Rijksinstituut voor Volksgezondheid en Milieu.)

\section{Chlamydia trachomatis infection}

$\mathrm{CT}$ is a gram negative obligate intracellular bacterium. ${ }^{8}$ This means that $\mathrm{CT}$ bacteria need host-epithelial cells to survive and to replicate. Four species of CT are well known to be pathogenic in humans, that are Chlamydia psittaci, Chlamydia abortus, Chlamydia pneumoniae and Chlamydia trachomatis. ${ }^{8}$ The studies in this thesis focusses on the latter, which is mainly transmitted via sexual contact with an infected partner (horizontal transmission), but can also be transmitted via mother to child during birth (vertical transmission).

To date, nineteen CT serovars have been identified. ${ }^{9}$ Serovars A-C can cause trachoma, which are ocular infections leading to blindness in mainly tropical areas. ${ }^{89}$ Serovars D-K can cause anogenital CT infections and are sexually transmittable. ${ }^{9}$ Serovars L1-L3 cause lymphogranuloma venereum (LGV) and are also sexually transmittable mainly in MSM and are more severe than serovars $D-K^{9}{ }^{9}$

The lifecycle of CT consists of two stadia: elementary bodies (EB) and reticulate bodies (RB). ${ }^{8}$ EBs are infectious and metabolically inactive, whereas RBs are not infectious and metabolically active. ${ }^{8}$ The EB form of CT invades host epithelial cells. ${ }^{8}$ Within the cell, the EBS transform into a intracellular form; the RBs. The RBs replicate within the host epithelial cell and transform back to the EBs. ${ }^{8}$ Eventually, the EBs are released and infect new host epithelial cells. ${ }^{8}$ The whole process can take 48-72 hours. The lifecycle of CT is depicted in Figure 2. 


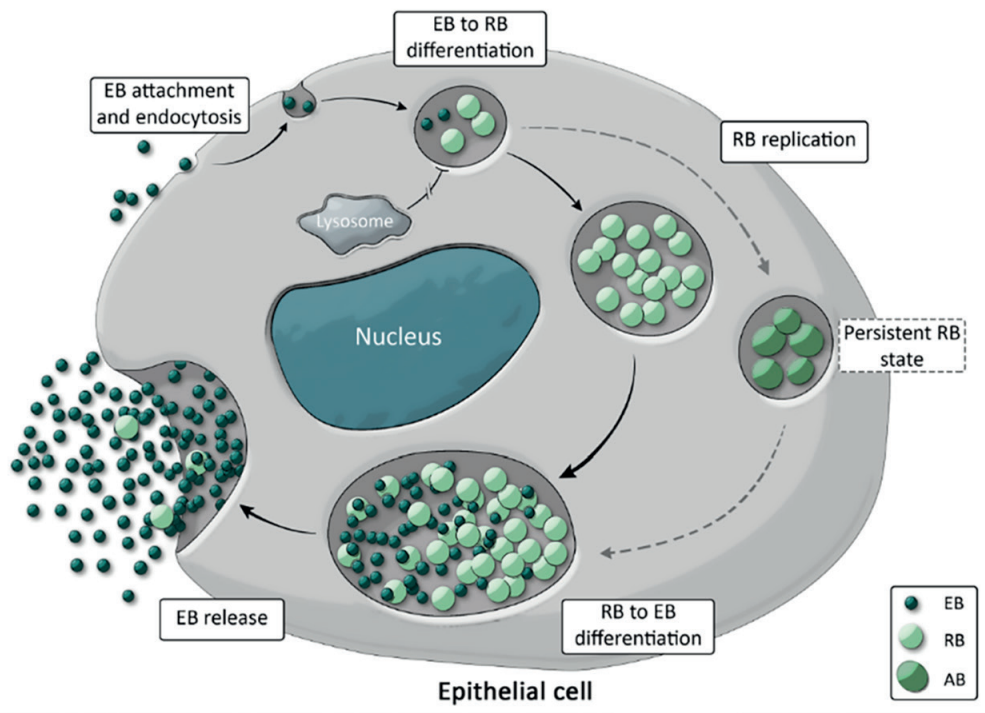

Figure 2. The lifecycle of a CT bacterium. EB, Elementary body; RB, Reticulate body; AB, Aberrant body. [Design: M. Lucchesi]

\section{Chlamydia trachomatis anatomic site distribution}

The majority of $\mathrm{CT}$ infections are asymptomatic in both men and women ranging from $31.2 \%$ to $100 \% .{ }^{10}$ Eventually, the asymptomatic nature of CT hampers early diagnosis and treatment and maintain ongoing transmission. CT infections can reside on different anatomic locations: the urogenital, anorectal or oropharyngeal site. CT test positivity rates differ per anatomic location and sex. Data from Dutch STI clinics show 14\% CT test positivity on the urogenital site in women, followed by $12 \%$ at the anorectal site and $2 \%$ at the oropharyngeal site. In heterosexual men, $18 \%$ test positive on the urogenital site, $6 \%$ on the anorectal site and $1 \%$ on the oropharyngeal site. In MSM numbers are slightly different, i.e. 3\% test positive on the urogenital site, $8 \%$ test positive on the anorectal site and $1 \%$ on the oropharyngeal site. ${ }^{5}$

\section{Transmission of Chlamydia trachomatis}

CT can be transmitted via sexual contact of different anatomic locations. The incubation period of CT is usually between 7-14 days. The most common routes of CT transmission are penile-vaginal, penile-anorectal, and oral sex. ${ }^{11-14}$ Anorectal CT in MSM is mainly transmitted via condom-less anorectal sex. ${ }^{11}$ Whereas the vast majority (71\%) of women with urogenital CT also have an anorectal CT infection irrespective of anorectal indications (i.e. no reported anal symptoms, no anal sex, and no anal use of fingers/toys). ${ }^{15}$ Current testing guidelines for anorectal CT advocate selective symptom- and sexual based testing for women. ${ }^{15}$ Therefore, 
undiagnosed anorectal CT infections in women can act as a reservoir and maintain ongoing transmission and potential sequelae. Some suggest that anorectal infections in women could be a result of auto-inoculation due to the close proximity of the vaginal and anorectal site. ${ }^{16}$ Several epidemiological studies have observed an association between other sexual activities such as fingering, fisting and rimming, and acquiring CT. ${ }^{11}$ Moreover, even saliva could play a role in transmitting CT, suggesting that CT can also be transmitted via kissing. ${ }^{11}$ Although, further research is needed to assess this pattern.

The number of CT positives from Dutch STI clinics is distributed over different transmission groups (50\% women, 28\% heterosexual men, and 23\% MSM). ${ }^{5}$ Therefore, CT is a more population-based disease affecting mostly the young general population. In contrast, the vast majority of NG positives cases in the Netherlands are from MSM (76\%), compared to women (16\%) and heterosexual men (9\%)..$^{5}$ Therefore, NG is mainly transmitted in the subpopulation of MSM. Repeat CT infections are common among patients earlier infected with CT. Patients with repeat CT infections could have disproportional impact on STIs within the population. ${ }^{17}$ It remains unknown which characteristics are associated with patients repeatedly infected with CT over several years. Insight in this high risk group could be useful to inform CT control and policy.

\section{Treatment of Chlamydia trachomatis}

According to international guidelines, uncomplicated urogenital CT infections are treated with 1 gram of azithromycin. ${ }^{318} 19$ Anorectal CT infections are treated with $100 \mathrm{mg}$ doxycycline twice daily for 7 days. ${ }^{18}{ }^{19}$ A study of our research group have shown that the treatment effectiveness of doxycycline exceeds that of azithromycin in anorectally CT positive women. ${ }^{20}$ There is an ongoing debate about whether all women should routinely be treated with doxycycline instead of azithromycin, since anorectal infections among women are common. ${ }^{13}$ Routine use of doxycycline would also treat undiagnosed anorectal infections and halt potential transmission even when anorectal CT infections are not tested for. However, a counter argument is that doxycycline should be taken for 7 days what could affect treatment adherence leading to not fully treated anorectal CT and potentially ongoing transmission of CT. ${ }^{21}$ Nevertheless, compliance has been shown to be positively influenced by good instructions and reminder apps.

\section{Chlamydia trachomatis bacterial load}

The CT bacterial load (hereafter: CT load), often expressed as the number of bacteria/mL, could potentially affect transmission of the disease and sequela. The CT load can be measured by samples (urine or swabs) taken from CT infected patients. Several studies have been 
performed assessing patient characteristics, symptoms, and transmission in relation to CT load. ${ }^{22}$ Most studies show inconsistent results, probably due to the low number of cases included and the different methods that are used to quantify CT load. ${ }^{23}$

Earlier, our study group showed that patients visiting the STI clinic and patients from a population based screening study (Chlamydia Screening Intervention [CSI]) have a similar CT load arguing for similar transmission potential and sequelae. ${ }^{24}$ It remains unknown whether patients visiting different STI care providers, such as the GP and STI clinic, have a different CT load. Insight in the CT load of patients visiting different STI care providers could be useful for public health purposes, since patients with a higher CT load could have a higher transmission potential.

CT on different anatomic locations could have different impact on transmission and sequelae. Several studies have assessed the CT load of different anatomic locations and sample types. ${ }^{225-28}$ However, those studies used different methods to quantify and report CT load making them less comparable. Moreover, no study compared the CT load of all relevant anatomic locations in one study using the same methods in men and women.

\section{Neisseria gonorrhoeae}

\section{Epidemiology of Neisseria gonorrhoeae in the Netherlands}

Similar to CT, GPs and STI clinics test and diagnose most NG patients, mostly simultaneously with CT as commercial tests assays provide both test at the same diagnostic platform. Approximately 9,550 patients were diagnosed with NG by GPs in 2017, this is 6.5\% more than in the year $2016 .^{5}$

STI clinics diagnosed 7,362 patients with NG in the year $2018 .{ }^{5}$ Among all NG patients in the Netherlands the vast majority are MSM (75.5\%), followed by women (15.7\%) and heterosexual men (8.8\%). ${ }^{5}$ The test positivity rates of NG are stable; among MSM 11.2\% tested positive in 2018 compared to $11.0 \%$ in $2017 .{ }^{5}$ Among heterosexual men $2.0 \%$ tested positive in 2018 and $1.9 \%$ in 2017. Among women, 1.7\% tested positive in 2018 in comparison with 1.6\% in 2017 (Figure 3). ${ }^{5}$

\section{Neisseria gonorrhoeae infection}

The bacterium NG is an aerobe gram negative diplococcus. ${ }^{29}$ The viability of the bacterium is sensitive to environmental change like acidity, temperature and (de)hydration. After trans- 
mission, NG affects the mucosal epithelium to replicate and to transmit to new host cells. ${ }^{29}$ NG mainly affect the urogenital mucosa, but is also colonize ocular, nasopharyngeal and anorectal mucosa. ${ }^{29}$

Of the genus Neisseria, NG and Neisseria meningitis are pathogenic. NG is mainly transmitted via sexual contact (horizontal transmission), but can also be transmitted via mother to child (vertical transmission). The latter can lead to neonatal blindness. ${ }^{29}$ Moreover, untreated NG can lead to disseminated NG infections potentially increasing the risk of infectious arthritis and endocarditis. ${ }^{29}$

NG infections can occur asymptomatic, but is sex specific. In one study, 78\% of MSM reported typical discharge. ${ }^{30}$ Overall, NG infections in women are more often asymptomatic compared to men. ${ }^{2931} 32 \mathrm{~A}$ reason for the difference between both sexes could rely on the fact that symptoms in men are easier to diagnose and specific, whereas symptoms, such as vaginal discharge, could be a result of other bacteria, such as bacterial vaginosis. ${ }^{29}$

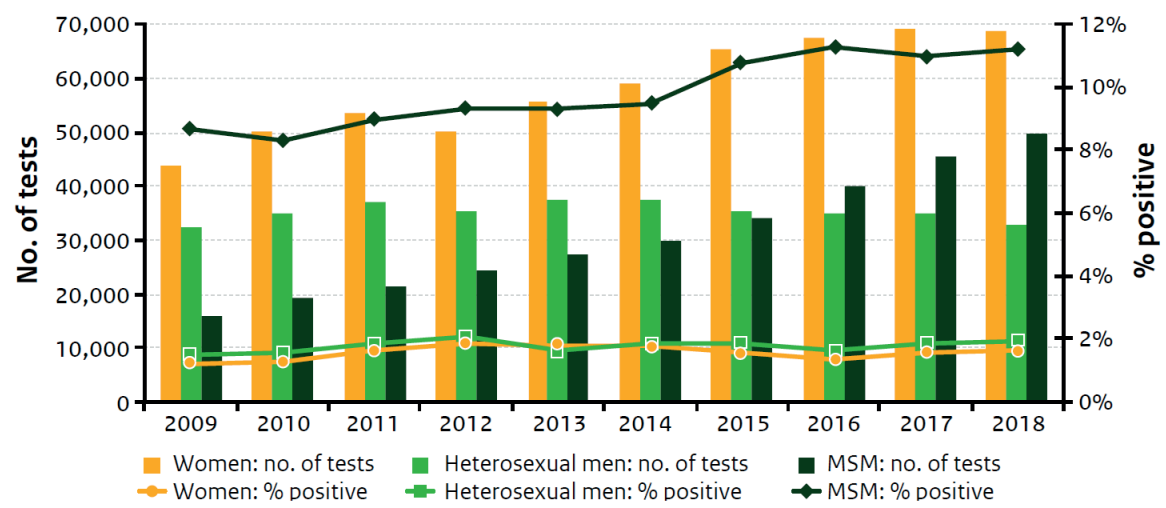

Footnote 1: Between 2012 and 2015, attendees below the age of 25 years with no further risk factors were only tested for chlamydia. Since 2015, attendees below the age of 25 years with no further risk factors were tested for chlamydia and gonorrhoea.

Footnote 2: Aggregated data of non-registered consultations included.

Figure 3. Total number of tests and positivity rate of gonorrhea by gender and type of sexual contact, 2009-2018

(Source: Slurink I, van Aar F, Op de Coul E, et al. Sexually transmitted infections in the Netherlands in 2018: Rijksinstituut voor Volksgezondheid en Milieu.)

\section{Neisseria gonorrhoeae anatomic site distribution}

Like CT, NG can reside on different anatomic locations, that is on the urogenital, anorectal or oropharyngeal site. According to recent numbers of The Netherlands National Institute for Public Health and the Environment, NG positivity rates of each anatomic location were for women: $1.3 \%$ urogenital, $1.5 \%$ anorectal, and $1.4 \%$ oropharyngeal. ${ }^{5}$ For heterosexual men 
this is: $1.8 \%$ urogenital, 3.1\% anorectal, and $0.0 \%$ oropharyngeal. ${ }^{5}$ The highest NG positivity rates are observed among MSM per anatomic location, that is $2.7 \%$ urogenital, $7.7 \%$ anorectal, and $5.7 \%$ oropharyngeal. ${ }^{5}$

\section{Transmission of Neisseria gonorrhoeae}

NG can be transmitted via sexual contact of different anatomic locations. NG is transmitted via penile-vaginal, penile-anal, and oral sex. ${ }^{11} \mathrm{NG}$ attaches to sperm and is mainly transmitted from men to their partners. ${ }^{29}$

In contrast to CT, anorectal NG is highly common among MSM. Condomless anorectal sex is a strong risk factor for anorectal NG. ${ }^{11}$ Other factors may also play a role in anorectal NG. For example, a study did not observe a difference in anorectal NG between MSM who had receptive penile-anal sex (8.8\%) and MSM who did not (6.6\%). ${ }^{33}$ This could indicate that other factors, such as receptive fingering, may also play a role the transmission of anorectal NG. ${ }^{11}$ Oropharyngeal NG is mainly transmitted via condomless oral-genital contact. A study reported that $73 \%$ of MSM had unprotected oral-genital contact with their last male partner. ${ }^{34}$ There is discussion about whether saliva could transmit NG via kissing. This transmission route is poorly understood and should be investigated further. ${ }^{11}$

NG is mainly transmitted in the sub-population of MSM and repeat NG infections are common. Therefore, testing, retesting and treating this high risk population is key for optimizing NG control. A study showed that patients repeatedly infected with any STI (CT, NG or syphilis) could have high impact on STI transmission within the population. ${ }^{17}$ Patients with repeat NG infections could be members of, so called, "core-groups" who continue NG transmission within the population. ${ }^{17}$ It remains unclear what socio-demographic characteristics are associated with patients who are repeatedly infected with NG over several years. Identifying the characteristics of this group could inform NG test practices and enhance NG control.

\section{Treatment of Neisseria gonorrhoeae}

International guidelines suggest as primary treatment for NG ceftriaxone 500mg intramuscular. ${ }^{35-37}$ When CT is not ruled out, a combination with $1000 \mathrm{mg}$ azithromycin (orally) should be given. ${ }^{35} 36$ Due to multidrug resistance, NG is becoming a widespread public health epidemic. In the 1940's NG was treated with penicillin, followed by spectinomycin, tetracycline, azithromycin, cefixime, and ceftriaxone until now. ${ }^{38}$ In recent years, ceftriaxone resistant NG cases have been identified in Canada, Japan, France, and Spain. ${ }^{39} 40$ No multidrug resistant NG cases are diagnosed in the Netherlands yet. ${ }^{5}$ Nevertheless, we expect it is a matter of time for the first multidrug resistant case in the Netherlands will arise. 


\section{(Inter)national Chlamydia trachomatis \& Neisseria gonorrhoeae testing guidelines}

Testing and treating individuals at high risk for STIs is key for effective control efforts as it reduces the infectious period of these infectious diseases. Moreover, diagnosis and treatment will lead to reduced sequelae on the individual level. Main international testing guidelines are provided by the Centers for Disease Control (CDC), the British Association for Sexual Health and HIV (BASHH), "the European guideline on the diagnosis and treatment of gonorrhoeae in adults", and "the European guideline on the management of Chlamydia trachomatis infections". 3193637

Individuals at risk for STIs should always be tested for urogenital CT and NG. Those individuals include patients younger than 25 year of age, MSM, commercial sex workers, patients originating from STI endemic countries, individuals with different sexual partners, individuals with a partner from earlier mentioned groups, and individuals notified by infected partners. ${ }^{35} 364142$ Additional extragenital testing should be performed based on indication. Dutch guidelines recommend anorectal testing in those with anorectal symptoms and/or anorectal sex. ${ }^{43}$ Oropharyngeal testing should be performed in those with self-reported oral sex or symptoms. ${ }^{43}$

STI clinics are subsidized by the Ministry of Health, Welfare and Sport to maintain free anonymous easy access testing for high risk groups. ${ }^{5}$ Whereas the general population at lower risk for STIs are meant to be tested by GPS. STI tests at the GP are within patients' deductibles in healthcare insurances what could be a barrier for testing. ${ }^{44}$

Earlier publications of our research group, using data until the year 2010, showed that GPs rarely test on extragenital locations. ${ }^{45} 46$ Unfortunately, suboptimal testing could lead to undiagnosed extragenital CT and NG infections and thus ongoing transmission. It remains unclear whether extragenital testing rates of GPs have been increased in recent years.

A CT or NG test-of-cure (TOC), which is a test taken 2 or 3 weeks after treatment to identify potential treatment failure, is not needed in uncomplicated CT or NG infections. ${ }^{3}$ A positive TOC could be false-positive due to recurrent non-viable CT or NG bacteria leading to overtreatment. ${ }^{47}$ A TOC should be performed in pregnant patients, in patients treated with an alternative medicine, or in patients with remaining symptoms. ${ }^{3}$ It remains unclear to what extent GPs perform a TOC for CT and NG. Insight in TOC test practices of GPs could be used to inform CT and NG control.

International guidelines recommend that all patients who are infected with CT or NG should be retested within 3 to 12 months independent of sexual behavior or sex. ${ }^{319} 363748$ Repeat CT or NG infections within 3 to 12 months of a previous CT or NG are common. ${ }^{49} 50$ According to systematic reviews, up to $32 \%$ of the CT patients and $40 \%$ of the NG patients have a repeat infection within one year. ${ }^{49}{ }^{50}$ Retesting CT and NG positives within 3 to 12 months is an effective control strategy to enhance population-based prevention of $\mathrm{CT}^{3}{ }^{3}$ 
Patients visiting STI clinics are actively informed to retest within 3 to 12 months. For the GP population this remains unknown. An earlier publication of our study group showed that retesting rates of GPs are relatively low. ${ }^{51}$ Retesting CT or NG patients within 3 to 12 months was also not advised in the Dutch national guideline for GPs until the year $2013 .{ }^{52}$ It remains unknown whether GP retesting rates have been increased after the guideline revision in recent years.

\section{Laboratory data of Maastricht University (MUMC+)}

The majority of studies in this thesis included laboratory data from the Medical Microbiology department of Maastricht University Medical Centre (MUMC+). This unique dataset included STI tests and test results from STI care providers in areas surrounding and including Maastricht, the Netherlands. Main STI care providers in the dataset included the STI clinic, GPS and hospital physicians. In this thesis, we included STI tests from GP practices located in Maastricht, Eijsden-Margraten and Valkenburg aan de Geul, since the vast majority (81\%) of these GP practices send their CT and NG tests to the microbiology laboratory of Maastricht University to ensure acceptable laboratory coverage. The total dataset include STI tests from January 2006 until July 2018 ( $n=204,479$ consultations).

This dataset has been created by the close collaboration of the Public Health Service South Limburg (GGD Zuid Limburg) and the Medical Microbiology department of MUMC+.

\section{Outline of this thesis}

In this thesis, epidemiological methods were used to analyze existing laboratory- and national surveillance CT and NG data. The results have led to new insights which can be used to inform and optimize CT and NG control.

The first section of this thesis covers repeat CT and NG testing and repeat infections. In chapter 2, TOC, retesting, extragenital test practices of GPS were analyzed. In chapter 3 and chapter 4, the characteristics of patients repeatedly infected with CT or NG were identified.

The second section of this thesis included studies assessing the CT load of different patients and anatomic locations. In chapter 5 the CT load of patients visiting different STI care providers, that is the STI clinic, GPs and hospital physicians, were assessed and compared.

Chapter 6 assessed the CT load of different sample types of men and women in one study using the same methods. In chapter $\mathbf{7}$, the CT load of urogenital and extragenital samples of patients repeatedly infected with CT was assessed.

In the last section two methodologies were compared for public health purposes. In chapter $\mathbf{8}$ an epidemiological analysis was compared with a geographical cluster analysis using Dutch national surveillance STI clinic data.

Finally, in chapter $\mathbf{9}$, the main findings and implication of this thesis are discussed. 


\section{References}

1. Newman L, Rowley J, Vander Hoorn S, et al. Global Estimates of the Prevalence and Incidence of Four Curable Sexually Transmitted Infections in 2012 Based on Systematic Review and Global Reporting. PLoS One 2015;10(12):e0143304. doi: 10.1371/journal.pone.0143304.

2. Haggerty CL, Gottlieb SL, Taylor BD, et al. Risk of sequelae after Chlamydia trachomatis genital infection in women. J Infect Dis 2010;201 Suppl 2:S134-55. doi: 10.1086/652395.

3. Workowski KA. Centers for Disease Control and Prevention Sexually Transmitted Diseases Treatment Guidelines. Clin Infect Dis 2015;61 Suppl 8:S759-62. doi: 10.1093/cid/civ771.

4. Costa-Lourenco A, Barros Dos Santos KT, Moreira BM, et al. Antimicrobial resistance in Neisseria gonorrhoeae: history, molecular mechanisms and epidemiological aspects of an emerging global threat. Braz J Microbio/ 2017;48(4):617-28. doi: 10.1016/j.bjm.2017.06.001.

5. Slurink I, van Aar F, Op de Coul E, et al. Sexually transmitted infections in the Netherlands in 2018: Rijksinstituut voor Volksgezondheid en Milieu.

6. Kampman C, Koedijk F, Driessen-Hulshof H, et al. Retesting young STI clinic visitors with urogenital Chlamydia trachomatis infection in the Netherlands; response to a text message reminder and reinfection rates: a prospective study with historical controls. Sex Transm Infect 2016;92(2):124-9. doi: 10.1136/sextrans-2015-052115.

7. Gotz HM, Wolfers ME, Luijendijk A, et al. Retesting for genital Chlamydia trachomatis among visitors of a sexually transmitted infections clinic: randomized intervention trial of home- versus clinicbased recall. BMC Infect Dis 2013;13:239. doi: 10.1186/1471-2334-13-239.

8. Elwell C, Mirrashidi K, Engel J. Chlamydia cell biology and pathogenesis. Nat Rev Microbiol 2016;14(6):385-400. doi: 10.1038/nrmicro.2016.30.

9. Dhawan B, Makharia GK, Juyal D, et al. Chlamydia trachomatis proctitis masquerading as carcinoma rectum: First case report from India. Indian journal of pathology \& microbiology 2017;60(2):259-61. doi: 10.4103/ijpm.ijpm_114_16 [published Online First: 2017/06/21].

10. Detels R, Green AM, Klausner JD, et al. The incidence and correlates of symptomatic and asymptomatic Chlamydia trachomatis and Neisseria gonorrhoeae infections in selected populations in five countries. Sex Transm Dis 2011;38(6):503-9.

11. Chow EP, Fairley CK. The role of saliva in gonorrhoea and chlamydia transmission to extragenital sites among men who have sex with men: new insights into transmission. J Int AIDS Soc 2019;22 Suppl 6:e25354. doi: 10.1002/jia2.25354.

12. Lau A, Kong FYS, Huston W, et al. Factors associated with anorectal Chlamydia trachomatis or Neisseria gonorrhoeae test positivity in women: a systematic review and meta-analysis. Sex Transm Infect 2019;95(5):361-67. doi: 10.1136/sextrans-2018-053950.

13. Chan PA, Robinette A, Montgomery M, et al. Extragenital Infections Caused by Chlamydia trachomatis and Neisseria gonorrhoeae: A Review of the Literature. Infect Dis Obstet Gynecol 2016;2016:5758387. doi: 10.1155/2016/5758387. 
14. Cornelisse VJ, Sherman CJ, Hocking JS, et al. Concordance of chlamydia infections of the rectum and urethra in same-sex male partnerships: a cross-sectional analysis. BMC Infect Dis 2017;17(1):22. doi: 10.1186/s12879-016-2141-7.

15. van Liere GA, Hoebe CJ, Wolffs PF, et al. High co-occurrence of anorectal chlamydia with urogenital chlamydia in women visiting an STI clinic revealed by routine universal testing in an observational study; a recommendation towards a better anorectal chlamydia control in women. BMC Infect Dis 2014;14:274. doi: 10.1186/1471-2334-14-274.

16. Heijne JCM, van Liere GAFS, Hoebe CJPA, et al. What explains anorectal chlamydia infection in women? Implications of a mathematical model for test and treatment strategies. Sexually transmitted infections 2017;93(4):270-75. doi: 10.1136/sextrans-2016-052786 [published Online First: 12/16].

17. Hsu KK, Molotnikov LE, Roosevelt KA, et al. Characteristics of Cases With Repeated Sexually Transmitted Infections, Massachusetts, 2014-2016. Clin Infect Dis 2018;67(1):99-104. doi: 10.1093/ cid/ciy029.

18. Miller KE. Diagnosis and treatment of Chlamydia trachomatis infection. Am Fam Physician 2006;73(8):1411-6.

19. Lanjouw E, Ouburg S, de Vries HJ, et al. 2015 European guideline on the management of Chlamydia trachomatis infections. International journal of STD \& AIDS 2016;27(5):333-48. doi: 10.1177/0956462415618837.

20. Dukers-Muijrers N, Wolffs PFG, Vries $H$, et al. Treatment effectiveness of azithromycin and doxycycline in uncomplicated rectal and vaginal Chlamydia trachomatis infections in women: a multicentre observational study (FemCure). Clin Infect Dis 2019 doi: 10.1093/cid/ciz050.

21. Khosropour CM, Manhart LE, Colombara DV, et al. Suboptimal adherence to doxycycline and treatment outcomes among men with non-gonococcal urethritis: a prospective cohort study. Sex Transm Infect 2014;90(1):3-7. doi: 10.1136/sextrans-2013-051174.

22. Vodstrcil LA, Mclver R, Huston WM, et al. The Epidemiology of Chlamydia trachomatis Organism Load During Genital Infection: A Systematic Review. J Infect Dis 2015;211(10):1628-45. doi: 10.1093/ infdis/jiu670.

23. Dirks JAMC, Hoebe CJPA, van Liere GAFS, et al. Standardisation is necessary in urogenital and extragenital <em>Chlamydia trachomatis</em> bacterial load determination by quantitative PCR: a review of literature and retrospective study. Sexually Transmitted Infections 2019:sextrans-2018-053522. doi: 10.1136/sextrans-2018-053522.

24. Dirks JA, Wolffs PF, Dukers-Muijrers NH, et al. Chlamydia trachomatis load in population-based screening and STI-clinics: implications for screening policy. PLoS One 2015;10(3):e0121433. doi: 10.1371/journal.pone.0121433.

25. Jalal H, Verlander NQ, Kumar N, et al. Genital chlamydial infection: association between clinical features, organism genotype and load. J Med Microbiol 2011;60(Pt 7):881-8. doi: 10.1099/ jmm.0.028076-0. 
26. Michel CE, Sonnex C, Carne CA, et al. Chlamydia trachomatis load at matched anatomic sites: implications for screening strategies. J Clin Microbio/ 2007;45(5):1395-402. doi: 10.1128/JCM.0010007.

27. van Liere GA, Dirks JA, Hoebe CJ, et al. Anorectal Chlamydia trachomatis Load Is Similar in Men Who Have Sex with Men and Women Reporting Anal Sex. PLoS One 2015;10(8):e0134991. doi: 10.1371/ journal.pone.0134991.

28. van Rooijen MS, van der Loeff MF, Morre SA, et al. Spontaneous pharyngeal Chlamydia trachomatis RNA clearance. A cross-sectional study followed by a cohort study of untreated STI clinic patients in Amsterdam, The Netherlands. Sex Transm Infect 2015;91(3):157-64. doi: 10.1136/ sextrans-2014-051633.

29. Quillin SJ, Seifert HS. Neisseria gonorrhoeae host adaptation and pathogenesis. Nat Rev Microbiol 2018;16(4):226-40. doi: 10.1038/nrmicro.2017.169.

30. Ong JJ, Fethers K, Howden BP, et al. Asymptomatic and symptomatic urethral gonorrhoea in men who have sex with men attending a sexual health service. Clin Microbiol Infect 2017;23(8):555-59. doi: 10.1016/j.cmi.2017.02.020.

31. Edwards JL, Apicella MA. The molecular mechanisms used by Neisseria gonorrhoeae to initiate infection differ between men and women. Clin Microbiol Rev 2004;17(4):965-81, table of contents. doi: 10.1128/CMR.17.4.965-981.2004.

32. Walker CK, Sweet RL. Gonorrhea infection in women: prevalence, effects, screening, and management. Int J Womens Health 2011;3:197-206. doi: 10.2147/IJWH.S13427.

33. Passaro RC, Segura ER, Perez-Brumer A, et al. Body Parts Matter: Social, Behavioral, and Biological Considerations for Urethral, Pharyngeal, and Rectal Gonorrhea and Chlamydia Screening Among MSM in Lima, Peru. Sex Transm Dis 2018;45(9):607-14. doi: 10.1097/OLQ.0000000000000816.

34. Rosenberger JG, Reece M, Schick V, et al. Sexual behaviors and situational characteristics of most recent male-partnered sexual event among gay and bisexually identified men in the United States. J Sex Med 2011;8(11):3040-50. doi: 10.1111/j.1743-6109.2011.02438.x.

35. De Vries H, van Dam A, Bax C. Seksueel Overdraagbare Aandoeningen Multidisciplinaire Richtlijn Update 20182018 [Available from: www.soaaids.nl/nl/professionals/beroepsgroep/arts/ professioneel-handelen/de-specialist accessed 3-9-2018.

36. Lazaro N. Sexually Transmitted Infections in Primary Care 2013 (RCGP/BASHH) available at www. rcgp.org and www.bashh.org/guidelines [cited 201720 April].

37. Bignell C, Unemo M, European STIGEB. 2012 European guideline on the diagnosis and treatment of gonorrhoea in adults. International journal of STD \& AIDS 2013;24(2):85-92. doi: 10.1177/0956462412472837.

38. Unemo M, Shafer WM. Antimicrobial resistance in Neisseria gonorrhoeae in the 21st century: past, evolution, and future. Clin Microbiol Rev 2014;27(3):587-613. doi: 10.1128/CMR.00010-14.

39. Centers for Disease C, Prevention. CDC Grand Rounds: the growing threat of multidrug-resistant gonorrhea. MMWR Morb Mortal Wkly Rep 2013;62(6):103-6.

40. Lefebvre B, Martin I, DemczukW, et al. Ceftriaxone-Resistant Neisseria gonorrhoeae, Canada, 2017. Emerg Infect Dis 2018;24(2) doi: 10.3201/eid2402.171756. 
41. Gotz HM, van Bergen JE, Veldhuijzen IK, et al. A prediction rule for selective screening of Chlamydia trachomatis infection. Sex Transm Infect 2005;81(1):24-30. doi: 10.1136/sti.2004.010181.

42. Van Bergen J, Dekker J, Boeke J, et al. NHG-Standard Het soa-consult (eerste herziening). HUISARTS EN WETENSCHAP-NEDERLANDS- 2013;56(9):450-63.

43. van Liere GA, Hoebe CJ, Niekamp AM, et al. Standard symptom- and sexual history-based testing misses anorectal Chlamydia trachomatis and neisseria gonorrhoeae infections in swingers and men who have sex with men. Sex Transm Dis 2013;40(4):285-9. doi: 10.1097/OLQ.0b013e31828098f8

44. Shi L, Xie Y, Liu J, et al. Is out-of-pocket cost a barrier to receiving repeat tests for chlamydia and gonorrhoea? International journal of STD \& AIDS 2013;24(4):301-6. doi: 10.1177/0956462412472821 [published Online First: 2013/08/24]

45. den Heijer CD, van Liere GA, Hoebe CJ, et al. Who tests whom? A comprehensive overview of Chlamydia trachomatis test practices in a Dutch region among different STI care providers for urogenital, anorectal and oropharyngeal sites in young people: a cross-sectional study. Sex Transm Infect 2016;92(3):211-7. doi: 10.1136/sextrans-2015-052065.

46. den Heijer CDJ, Hoebe C, van Liere G, et al. A comprehensive overview of urogenital, anorectal and oropharyngeal Neisseria gonorrhoeae testing and diagnoses among different STI care providers: a cross-sectional study. BMC Infect Dis 2017;17(1):290. doi: 10.1186/s12879-017-2402-0.

47. Workowski KA, Bolan GA. Sexually transmitted diseases treatment guidelines, 2015. MMWR Recommendations and reports: Morbidity and mortality weekly report Recommendations and reports 2015;64(Rr-03):1-137. [published Online First: 2015/06/05]

48. Nwokolo NC, Dragovic B, Patel S, et al. 2015 UK national guideline for the management of infection with Chlamydia trachomatis. International journal of STD \& AIDS 2016;27(4):251-67. doi: 10.1177/0956462415615443

49. Fung M, Scott KC, Kent CK, et al. Chlamydial and gonococcal reinfection among men: a systematic review of data to evaluate the need for retesting. Sex Transm Infect 2007;83(4):304-9. doi: 10.1136/ sti.2006.024059.

50. Hosenfeld CB, Workowski KA, Berman S, et al. Repeat infection with Chlamydia and gonorrhea among females: a systematic review of the literature. Sex Transm Dis 2009;36(8):478-89. doi: 10.1097/OLQ.0b013e3181a2a933.

51. Dukers-Muijrers NH, van Liere GA, Hoebe CJ. Re-screening Chlamydia trachomatis positive subjects: a comparison of practices between an STI clinic, general practitioners and gynaecologists. Sex Transm Infect 2013;89(1):25-7. doi: 10.1136/sextrans-2011-050467.

52. Van Bergen JEAM, et al. Standard of the National Society for General Practice: the STI consultation [NHGStandaard: Het soa-consult]. Huisarts en Wetenschap 2013;56(9):450-63. 


Section retesting 


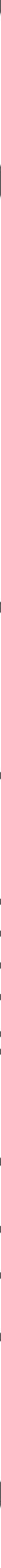





\section{Abstract}

\section{Background}

For Chlamydia trachomatis (CT), a test of cure (TOC) within 3-5 weeks is not recommended. International guidelines differ in advising a Neisseria gonorrhoeae (NG) TOC. Retesting CT and NG positives within 3-12 months is recommended in international guidelines. We assessed TOC and retesting practices including extragenital testing in general practitioner (GP) practices located in different socioeconomic status (SES) areas to inform and optimize local test practices.

\section{Methods}

Laboratory data of 48 Dutch GP practices between January 2011 and July 2016 were used. Based on a patient's first positive CT or NG test, the proportion of TOC ( $<3$ months) and retests (3-12 months) were calculated. Patient- and GP-related factors were assessed using multivariate logistic regression analyses.

\section{Results}

For CT ( $n=622$ ), 20\% had a TOC and 24\% had a retest at the GP practice. GP practices in low SES areas were more likely to perform a CT TOC (OR:1.8;95\% Cl:1.1-3.1). Younger patients (<25 years) were more likely to have a CT TOC (OR:1.6;95\% Cl:1.0-2.4). For CT ( $n=622$ ), 2.4\% had a TOC and $6.1 \%$ had a retest at another STI care provider. For NG ( $n=73), 25 \%$ had a TOC and $15 \%$ had a retest at the GP practice. For NG ( $n=73), 2.7 \%$ had a TOC and $12.3 \%$ had a retest at another STI care provider. In only $0.3 \%$ of the consultations patients were tested on extragenital sites.

\section{Conclusion}

Almost 20\% of the patients returned for a CT TOC, especially at GP practices in low SES areas. For NG, 1 out of 4 patients returned for a TOC. Retesting rates were low for both CT (24\%) and NG (15\%), (re)infections including extragenital infections may be missed. Efforts are required to focus TOC and increase retesting practices of GPs in order to improve CT/NG control. 


\section{Introduction}

Chlamydia trachomatis (CT) and Neisseria gonorrhoeae (NG) are the most prevalent bacterial sexually transmitted infections (STIS) diagnosed worldwide. ${ }^{1}$ General practitioners (GPS) have a major role in STI healthcare, since most STIs are diagnosed by GPS and STI clinics. ${ }^{2-6}$

A test of cure (TOC) within 3-5 weeks after the completion of CT treatment is internationally not recommended because of possible false-positive results leading to overtreatment. ${ }^{7-9}$ The British Association for Sexual Health and HIV in cooperation with the Royal College of General Practitioners, as well as the 2012 European guideline on the diagnosis and treatment of gonorrhoea in adults, recommend a TOC for all patients who tested positive for NG two weeks after treatment due to the increasing antimicrobial resistance of NG. ${ }^{10}{ }^{11}$ However, the American Centers for Disease Control and Prevention only recommend an NG TOC in the case of oropharyngeal NG when treated with an alternative regimen. ${ }^{9}$ The Dutch general practitioner guideline does not recommend a CT TOC nor an NG TOC, except for specifically indicated cases. ${ }^{12}$

Repeat infections within 3-12 months after CT/NG diagnoses are common, totaling up to 32\% for CT and up to 40\% for NG. ${ }^{13}{ }^{14}$ For effective CT/NG control, retesting CT/NG positives within 3-12 months is recommended by the Centers for Disease Control and Prevention as well as the British Association for Sexual Health and HIV. ${ }^{90}$ Moreover, a modeling study suggested that the most effective control strategy for the treatment of resistant NG is to retest NG positives, rather than testing and treating more patients. ${ }^{15}$ Furthermore, the Dutch GP guideline has been revised since 2013 and recommends that GPs advise CT-positive patients to consider retesting within a year. ${ }^{12}$ However, no recommendations are given for retesting NG-positive patients. ${ }^{12}$

The majority of CT and NG positives seen by GPs are not retested. ${ }^{16-18}$ However, retesting rates may have been underestimated, as patients could have a TOC or a retest at another STI care provider like the STI clinic or the hospital. ${ }^{16}$ Insight into TOC and retesting rates, including associated factors among GPs and patients, is vital to inform and improve CT and NG control.

Extragenital CT infections are common among men who have sex with men (MSM) and women. ${ }^{19}$ International guidelines recommend extragenital testing based on indication; that is, self-report of anal sex and symptoms. ${ }^{710}$ However, studies showed that extragenital CT infections are common among MSM and women without indication, which suggests routine screening at extragenital sites among MSM and women. ${ }^{19}{ }^{20}$ According to the Dutch GP guideline, additional anorectal CT/NG testing in MSM and an indication of anal sex or anal symptoms is recommended. ${ }^{12}$ For NG, additional oropharyngeal testing should be performed for commercial sex workers and MSM who report oral sex or oropharyngeal symptoms. According to recent studies among GPs in the Netherlands, extragenital CT and NG testing was rarely performed. ${ }^{218}$ However, an earlier Dutch study estimated that $~ 9 \%$ of male patients with a STI-related GP visit are MSM, for whom extragenital testing is recommended. ${ }^{1221}$ 
The socioeconomic status (SES) of patients has been linked to various infectious diseases like CT and NG. ${ }^{2}{ }^{1822}$ However, it remains unknown whether TOC and retesting practices differ between GP practices in low, middle and high SES areas. Such analyses that include the SES of GP practice areas could be helpful in advising GPS about their TOC and retest practices to optimize CT and NG control at a local level.

Here, we assessed CT and NG TOC and retesting practices of GP practices in different SES areas, taking account of TOC and retesting of patients at other STI care providers as well as extragenital testing, in order to inform and optimize local test practices of GP practices.

\section{Methods and materials}

\section{Ethics statement}

The data were obtained from medical records in a fully anonymized and de-identified manner and none of the researchers had access to patient identifying information. The study protocol was exempt from formal medical-ethical approval under prevailing laws in the Netherlands as it concerns an retrospective observational study using anonymous data only (as stated by the National Central Committee for Human Studies: www.ccmo.nl and in the conduct of good behavior in research www.federa.org).

\section{Study population}

All CT and/or NG laboratory tests of patients $\geq 16$ years were obtained from the database of the regional medical microbiology laboratory of Maastricht University Medical Center (January 2011-July 2016; $n=47,311$ ). The dataset consisted of tests from all four STI care providers, as shown in Figure 1. The study area comprised municipalities surrounding the regional laboratory (Maastricht, Eijsden-Margraten and Valkenburg aan de Geul).

For the purpose of this study, a GP practice was defined as one or more GPs sharing the same postal code and performing CT and/or NG tests. The study area consisted of 48 GP practices. We estimated whether GP practices in the study area sent their CT and NG tests to the regional laboratory. In case of low numbers of CT tests $(\leq 40)$ per GP practice, or a notable downward trend between 2011 and 2016, GP practices were contacted by telephone to confirm whether they indeed sent their test requests to the regional laboratory. We contacted 20 GP practices in our study area, of which 9 GP practices reported that they sent their tests to another laboratory. As a consequence, the data set covered 81\% (39/48) of the GP practices in our study area, ensuring acceptable laboratory coverage. All 48 GP practices were included in the analyses. 
Dutch SES scores based on income, education level and employment were extracted from the Netherlands Institute for Social Research (http://www.scp.nl) per four-digit postal code area. The SES score of the GP practice area was determined on the basis of the four-digit postal code of the GP practice. The SES score of the patients' residential area was based on the four-digit postal code of the patient.

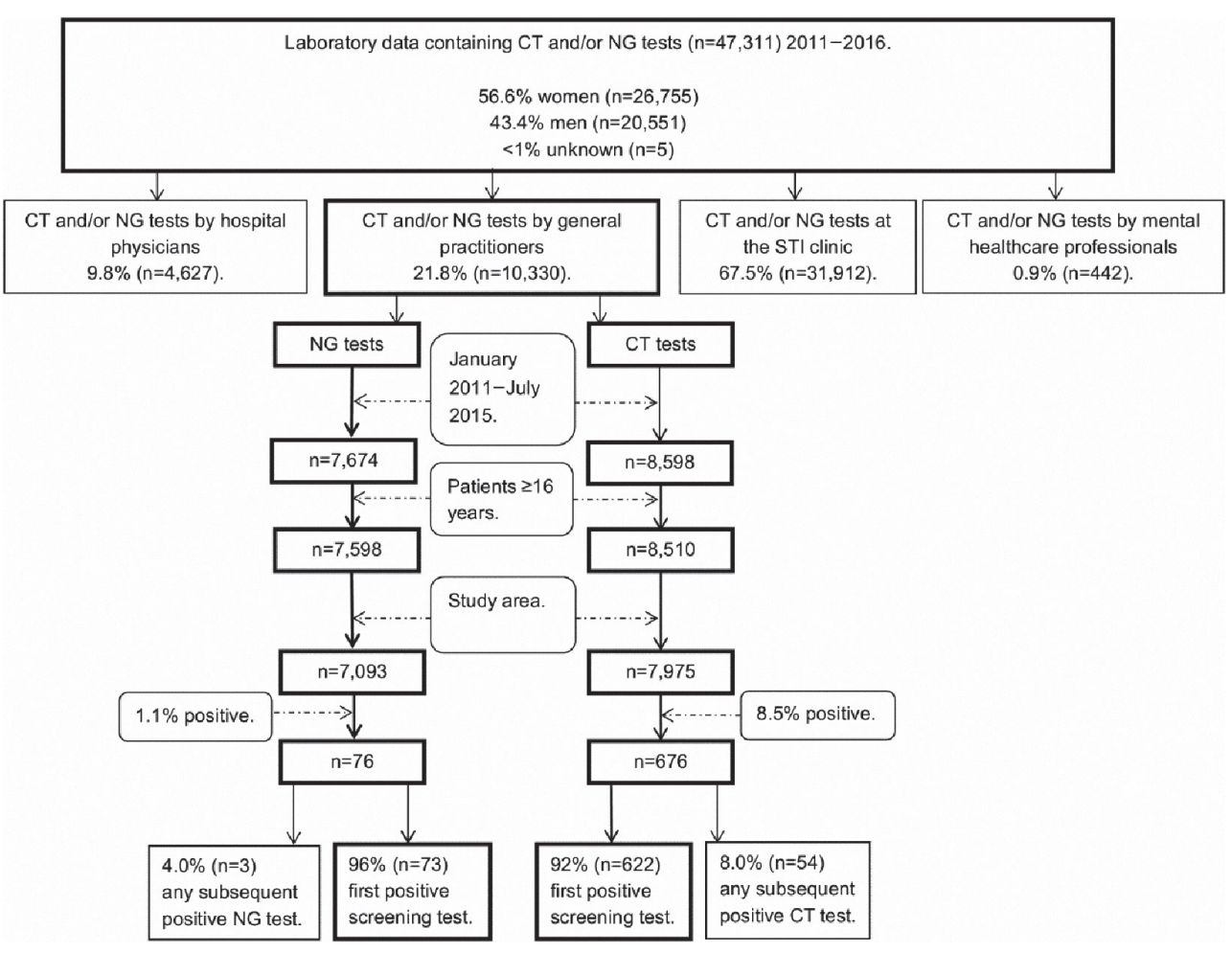

Figure 1. Flowchart of Chlamydia trachomatis- and Neisseria gonorrhoeae-positive screening tests between January 2011 and July 2015.

CT, Chlamydia trachomatis; NG, Neisseria gonorrhoeae; STI, sexually transmitted infection

\section{Definitions}

The first positive CT or NG test of a patient was defined as the positive screening test. For those patients identified as having a positive screening test, we assessed five outcome measures: (1) a 'TOC' was defined as the first test within three months of the positive screening test carried out by the same GP practice (the period of three months was based on international guidelines advising a retest three months after CT diagnosis ${ }^{9}{ }^{10}$ ); (2) 'TOC at another STI care provider' was defined as the first test within three months of the positive screening test carried by other STI care providers; that is, other GP practices, STI clinics or hospital 
physicians; (3) 'retesting' was defined as the first test within 3-12 months after the positive screening test carried out by the same GP practice; (4) 'retesting at another STI care provider' was defined as the first test within 3-12 months of the positive screening test carried out by other STI care providers; that is, other GP practices, STI clinics or hospital physicians and (5) 'extragenital testing' was defined as the proportion of consultations in which anorectal and oropharyngeal tests were performed by GPs in the period 2011-2015.

TOC and retesting rates were calculated per positive screening test. We excluded CT-positive ( $n=138$ ) and NG-positive ( $n=20)$ screening tests and any other tests of patients that were performed during the last year of the data collection (July 2015-July 2016) to ensure the same window of opportunity for retesting among all patients. Positive scores for TOC, retesting and extragenital testing were calculated by the proportion that tested positive at the first TOC or retest, or at extragenital locations.

\section{Study cohort and statistical analysis}

In the period of January 2011 to July 2015, there were 8,014 GP testing consultations. In 88\% $(n=7,054)$ of the consultations, patients were tested for both CT and NG; $11.5 \%(n=921)$ were tested for CT only and $0.5 \%$ ( $n=39)$ for NG only.

Analyses were stratified for positive CT and NG screening tests. This procedure resulted in a baseline cohort of 622 CT-positive and 73 NG-positive screening tests (Figure 1), in which every positive screening test in the analysis dataset represents one patient.

To assess factors associated with (1) TOC and (2) retesting, univariate and multivariate logistic regression analyses were performed. The factors assessed were sex, age $(<25, \geq 25)$, screening test result (tested for CT only, tested positive for CT and negative for NG, tested positive for CT and NG), TOC (yes, no) for the outcome retest only, SES of patients' residential area (low, medium, high SES, bases on tertiles), calendar year of screening $(<2013, \geq 2013$; the Dutch GP guideline was revised in 2013), number of tests on a GP practice level (continuous with an increment of 10 tests), urbanization of GP practice area (urban, non-urban), SES of GP practice area (low, medium, high SES, based on tertiles), distance between GP practice and STI clinic ( $<3 \mathrm{~km}, \geq 3 \mathrm{~km}$ ), distance between GP practice and laboratory $(<3 \mathrm{~km}, \geq 3 \mathrm{~km})$, distance between GP practice and patient ( $<3 \mathrm{~km}, \geq 3 \mathrm{~km}$ ), and number of employed GPs per GP practice $(1,>1)$.

Based on existing literature ${ }^{16-1823}$, multivariate analyses were adjusted for the following factors: sex, age and number of tests per GP practice. The SES of the GP practice area was also included in the multivariate model, as this factor was our main interest.

For all analyses, factors with $p<0.10$ in the univariate model were included through the backward stepwise method in the multivariate model. Odds ratios and 95\% confidence intervals $(95 \% \mathrm{Cl})$ were calculated for the univariate and multivariate models. Analyses were 
performed using SPSS V21 (IBM SPSS Statistics for Windows, IBM Corporation, Armonk, New York, USA). A p value of $<0.05$ was considered statistically significant.

\section{Results}

\section{Chlamydia trachomatis}

\section{TOC}

Of our baseline cohort (622 CT-positive patients), 19.6\% had a TOC within 3 months (122/622). Of this number, $15.6 \%$ tested positive (19/122; Figure $2 A)$. Of the patients with a positive TOC ( $n=19), 63.2 \%$ (12/19) tested positive within 3 weeks, whereas the minimum time for performing a CT TOC is at least 3 weeks..$^{7-9}$ The median time to a TOC was 36 days (interquartile range (IQR) 28-54).Factors independently associated with TOC in multivariate analyses were patients having a younger age ( $<25$ year) and GP practice areas with low and medium SES, respectively (Table 1 ).

\section{TOC at another STI care provider}

Of our baseline cohort, 2.4\% (15/622) had a TOC at another STI care provider (none at another GP practice, 11 at the STI clinic and 4 at the hospital). Of this number, 33.3\% (5/15) tested positive (Figure 3) 


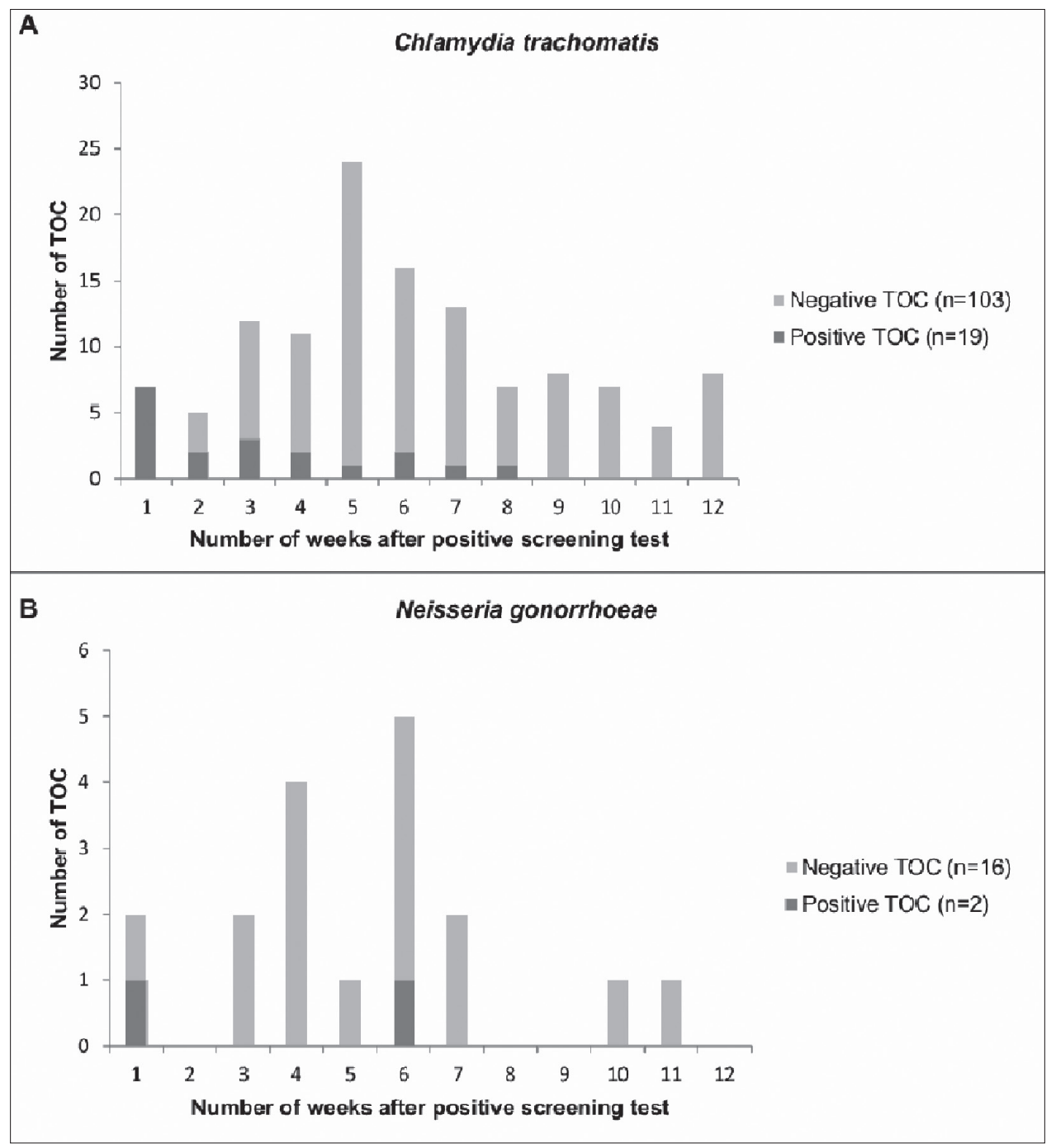

Figure 2. Distribution of the number of tests of cure and positivity per week.

(A) The number of tests of cure per week for Chlamydia trachomatis based on the positive screening test $(n=622)$. Two tests of cure were performed in week 13 , for clarity reasons these were added to week 12. (B) The number of tests of cure per week for Neisseria gonorrhoeae based on the positive screening test ( $n=73)$.

TOC, test of cure. 


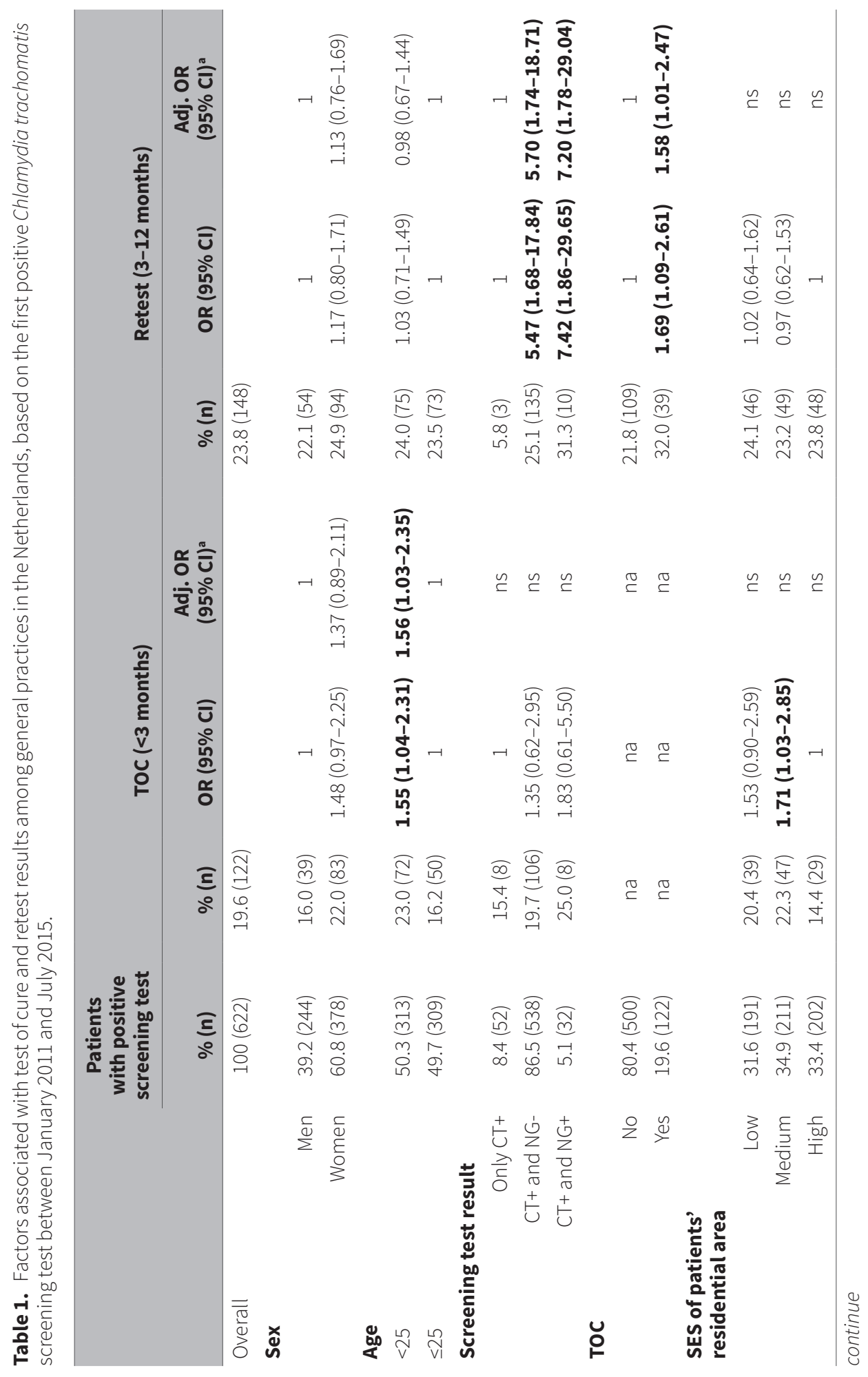




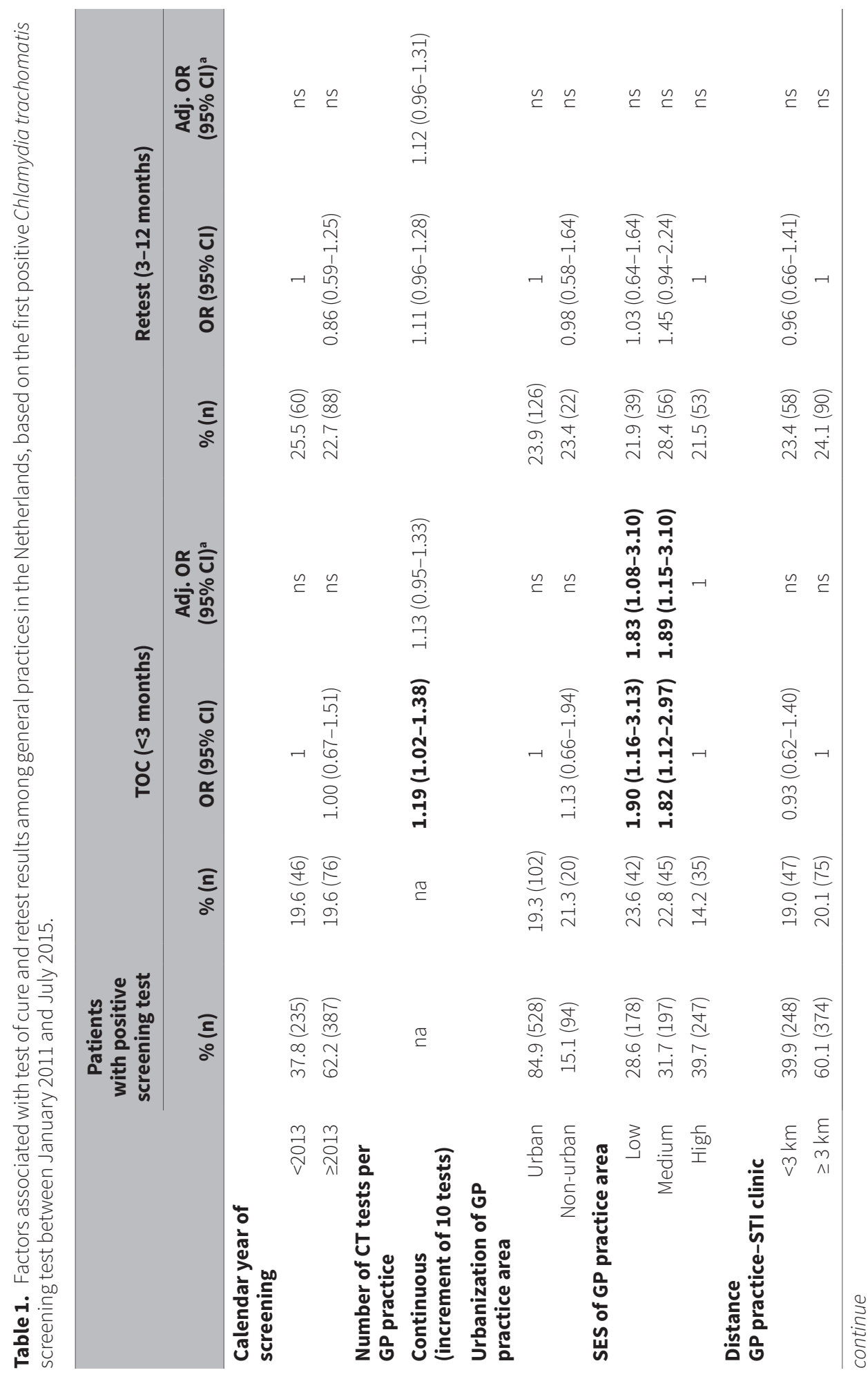




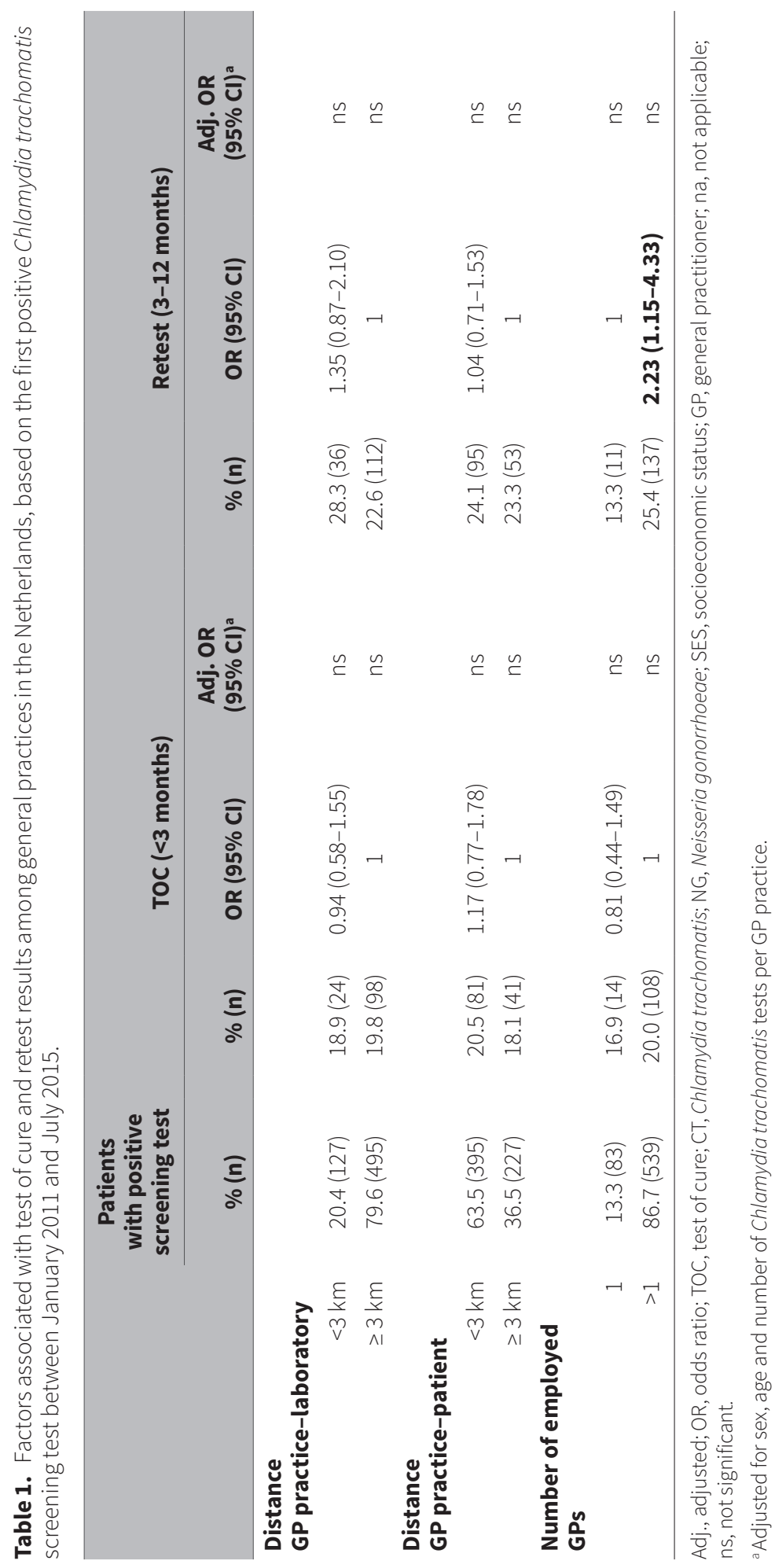




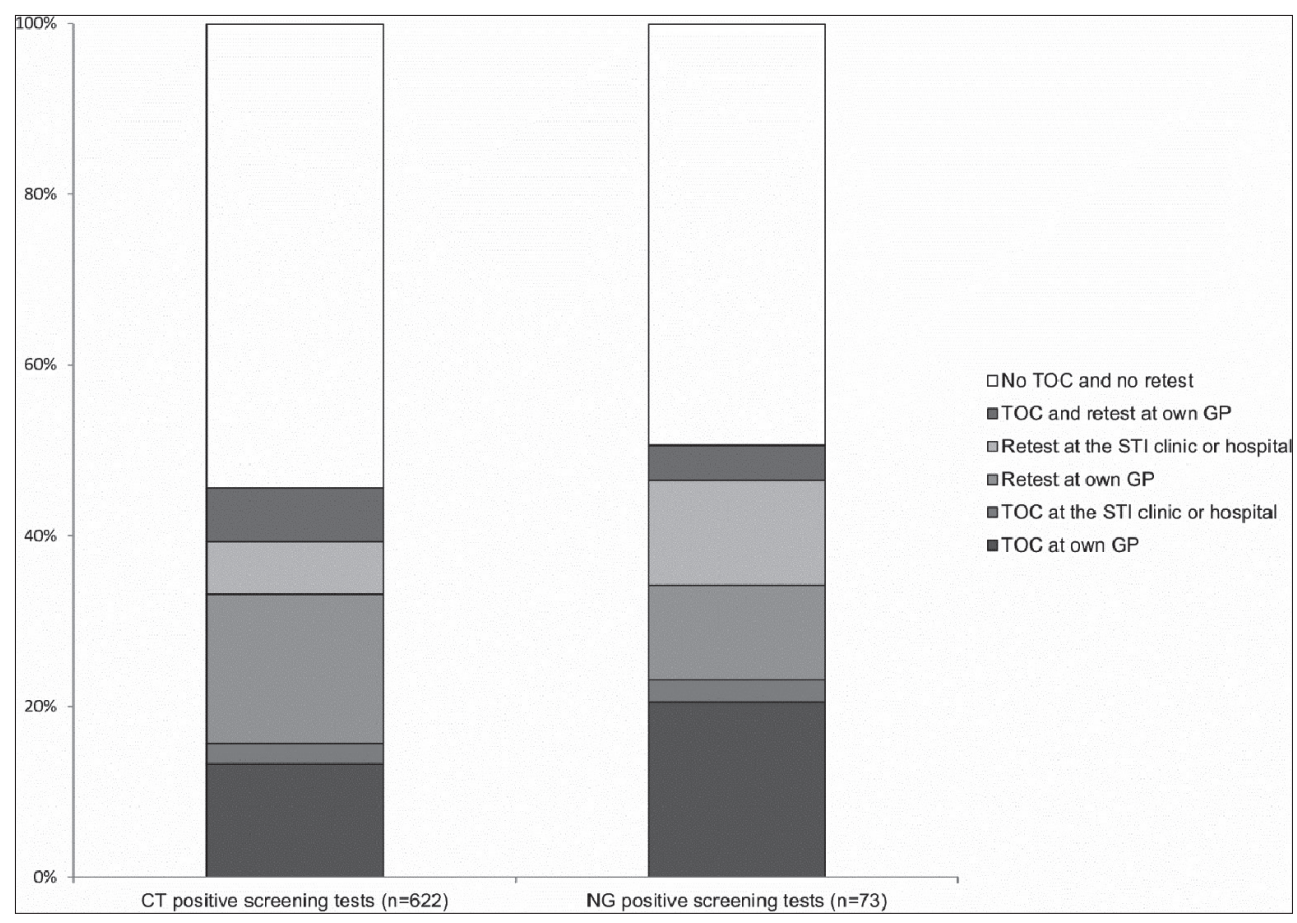

Figure 3. Distribution of Chlamydia trachomatis and Neisseria gonorrhoeae test of cure and retest results among patients with a positive screening test at the general practice.

CT, Chlamydia trachomatis; NG, Neisseria gonorrhoeae; TOC, test of cure; GP, general practice; STI, sexually transmitted infection.

\section{Retesting}

A retest within 3-12 months was performed in 23.8\% (148/622) of the CT patients. Of this number, $12.2 \%$ tested positive (18/148). The median time to a retest was 182 days (IQR $125-$ 265). Factors independently associated with retesting in multivariate analyses were patients who screened positive for CT and negative for NG, patients who screened positive for both CT and NG, and patients who had a TOC (Table 1).

\section{Retesting at another care provider}

Retesting at other STI care providers comprised 6.1\% (38/622) (none at another GP practice, 32 at the STI clinic and 6 at the hospital). Of this number, 15.8\% (6/38) tested positive (Figure 3). 


\section{Extragenital testing}

Testing at extragenital sites was performed in $0.3 \%$ of the CT consultations (25/7975). Of this number, 7 were on anorectal sites (positivity 14.3\%; 1/7) and 18 were on oropharyngeal sites (positivity 0\%; 0/18).

Neisseria gonorrhoeae

TOC

Of our baseline cohort of 73 patients, $24.7 \%$ had a TOC within 3 months (18/73). Of this number, $11.1 \%$ (2/18) tested positive. The median time to a TOC was 33 days (IQR 21-42). One patient had a positive TOC after 5 days of the positive screening test and one patient had a positive TOC after 41 days of the positive screening test (Figure 2B). In multivariate analyses, no factors were independently associated with TOC (Table 2). 


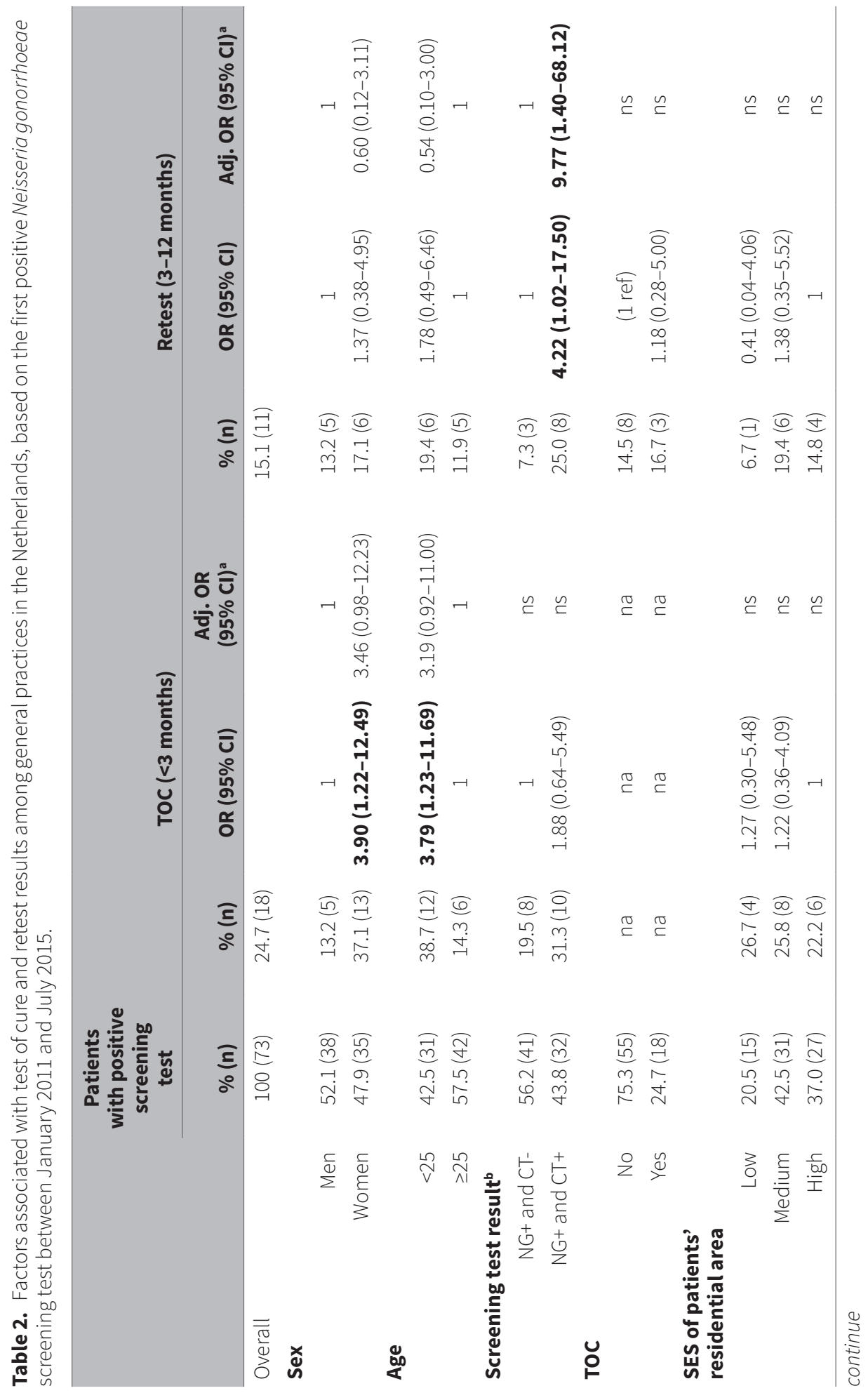




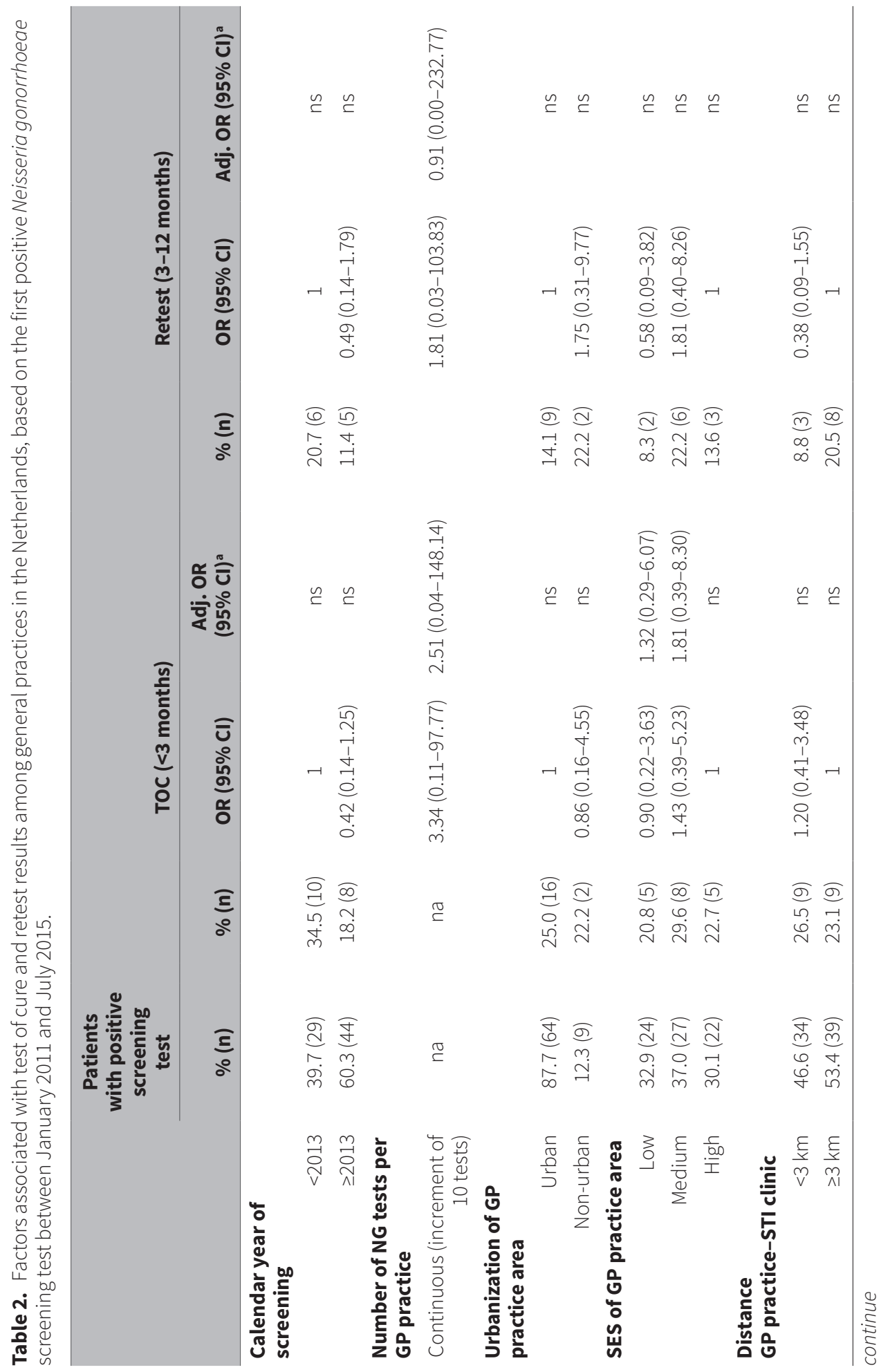




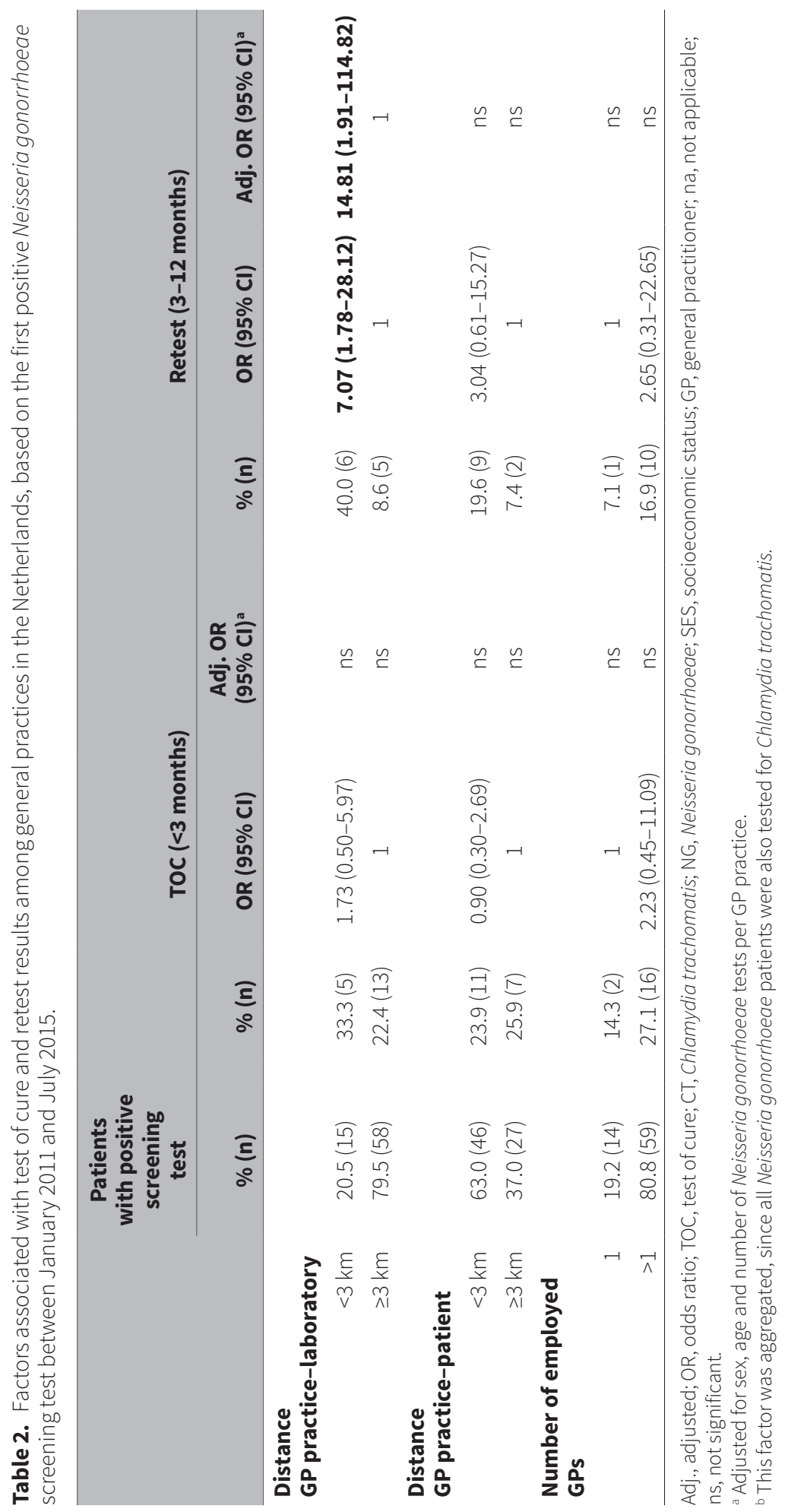




\section{TOC at another STI care provider}

Of the 73 patients with a positive NG screening test, 2.7\% (2/73) had a TOC at another STI care provider (two at the STI clinic). Of this number, one tested positive (Figure 3).

\section{Retesting}

A retest within 3-12 months was performed in 15.1\% of the NG patients (11/73). Of this number, 0\% (0/11) tested positive. The median time to a retest was 224 days (IQR 140-254). Independent factors associated with retesting in multivariate analyses were patients screening positive for both NG and CT, and a distance of $<3 \mathrm{~km}$ between GP practice and laboratory (Table 2).

\section{Retesting at another STI care provider}

In $12.3 \%$ (9/73) of the patients a retest was performed at another STI care provider (none at another GP practice, seven at the STI clinic and two at the hospital). Of this number, none tested positive (Figure 3).

\section{Extragenital testing}

Testing at extragenital sites was performed in $0.3 \%(23 / 7,093)$ of the NG consultations. Of this number, 6 were performed on anorectal sites (positivity 0.0\%; 0/6) and 17 on oropharyngeal sites (positivity 5.9\%; 1/17). 


\section{Discussion}

This retrospective cohort analysis of 48 Dutch GP practices shows that a TOC was performed in approximately $20 \%$ of the CT patients, especially at GP practices in low SES areas and in patients with a younger age (<25). Furthermore, 1 out of 4 patients had an NG TOC, which is generally not recommended in the Dutch GP guideline. However, an NG TOC is recommended in international guidelines due to increasing antimicrobial resistance of NG. ${ }^{9-11}$ Most CT (76\%) and NG (85\%) patients did not have a retest at their GP. Overall, 12\% retested CT positive and $0 \%$ retested NG positive. Due to the low number of CT and NG retests, repeat infections were likely missed. A comparable proportion of the NG patients were retested at the GP practice (15\%) and at other STI care providers (12\%), while the percentages for CT retests were $24 \%$ at the GP practice and 6\% at other STI care providers. Furthermore, extragenital CT and NG testing was rarely performed at GP practices (0.3\%), in which extragenital infections were likely missed.

Several studies have been performed assessing CT/NG test practices among different STI care providers. ${ }^{16}{ }^{23-26}$ However, these studies only include patient-related factors and lack GP characteristics. We included these factors to draw up recommendations specifically for GPs in order to enhance CT/NG control. Moreover, we provided evidence that the retesting rates of patients initially seen by their GP are underestimated; that is, we showed that the rate of retesting NG patients at other STI care providers (12\%) is comparable to the rate of retesting at the GP practice (15\%). Despite the small numbers, analyses of NG test practices are necessary, as following up on NG positives seems to be the most effective control strategy for the treatment of resistant NG. ${ }^{15}$ Furthermore, we analyzed recent data from before and after the revision to the Dutch GP guideline in order to assess current retesting practices of GPS. However, no difference between the two time periods was observed (Table 1 and 2). Finally, a further strength is that we assessed whether GP practices in our study area indeed send their tests to the regional laboratory, in which we estimated that $81 \%(39 / 48)$ of the GP practices did.

A general limitation of the study was that information on patients' sexual behavior and on the characteristics of or reasons for testing was unavailable. Such reasons may include financial reasons. STI tests at the GP are within patients' deductibles in healthcare insurances, whereas STI tests at the STI clinic are free of charge for risk groups (age <25, MSM and commercial sex workers). ${ }^{23}$ An earlier study in the southeastern part of Limburg showed that CT retesting rates were lower for GPS (23.0\%) in comparison with the STI clinic (33.4\%) and gynecologists (30.3\%). ${ }^{17}$ In addition, we operationalized the outcomes according to international guidelines with a cut-off point of three months. As a result, we were unable to assess whether a CT/NG TOC or retest was also defined as such by the treating GP. A limitation for the outcome TOC was that we were unable to assess whether a CT or NG TOC was justified according to the Dutch GP guideline; that is, in the case of pregnancy, persistent symptoms, reexposure to untreated source or lack of treatment with first choice of treatment. ${ }^{12}$ In addition, the use 
of the same time period for assessing TOC for both CT and NG is debatable. While a TOC for CT is not advisable, a TOC two weeks after NG treatment is internationally recommended. ${ }^{10-14}$ However, to enable a comparison between CT and NG, we applied equal TOC periods. ${ }^{12}$ Still, the exact time for performing an NG TOC is under debate. ${ }^{2829}$

We showed that almost 1 in every 5 patients had a CT TOC. Moreover, almost two-thirds of the positive CT TOC were diagnosed within 3 weeks of the positive screening test, which is strongly not recommended and can be false positive results leading to overtreatment. ${ }^{89}{ }^{30}$ If indicated, a CT TOC should be performed at least 3 weeks post treatment. ${ }^{79} 12$ An Australian study showed that 25\% of the patients who tested positive for CT at the GP made a new test request within six weeks, which is also not recommended in the Australian GP guidelines. ${ }^{16}$ An NG TOC was performed in almost 1 in 4 patients, which is not recommended in the Dutch GP guideline. However, forgoing a TOC after NG treatment is debatable, as international guidelines recommend a TOC two weeks post treatment for all NG patients ${ }^{10}{ }^{11}$ or patients with oropharyngeal NG and treated with alternative medication. ${ }^{9}$ One patient had a positive TOC after 40 days of the positive screening test, which could be related to treatment failure. ${ }^{11}$ Still, no cases of antimicrobial resistance have yet been reported in the Netherlands. ${ }^{15} 31$

A high proportion of CT repeat infections were diagnosed. Other studies showed higher retesting rates among GPs than our study, still retesting rates were low and likely infections were missed. ${ }^{1632}$ Moreover, due to low rate of extragenital testing also extragenital infections were likely missed, since extragenital infections are common in MSM as well as in women without symptoms in STI clinic settings. ${ }^{1921}$ However, this remains unclear for the GP population. Similar findings of low extragenital testing among GPs in another Dutch region confirm the likely generalizability of our study. ${ }^{218}$

According to the laboratory data (Figure 1), most CT and NG tests were performed by the STI clinic followed by GP practices. In the Netherlands, TOC rates are higher at GP practices compared to STI clinics, whereas retesting rates are higher at STI clinics compared to GP practices. ${ }^{17} 1823$ However, in other countries retesting rates seem higher at GP practices than STI clinics. ${ }^{1632}$

According to previous studies, the number of positive CT and NG tests is highest among patients living in low SES areas. ${ }^{218}$ The SES of patients' living areas was not associated with CT or NG TOC in the current study. However, most patients lived near the GP practice $(<3 \mathrm{~km}$; Table 1 and 2). This fact could explain the higher CT TOC rate of GP practices in low SES areas in our study. Our results show that patients who tested for both CT and NG were more likely to retest for CT and NG in comparison with patients who only tested for CT (Table 1). Patients without an NG test are likely to be a low-risk group or to lack symptoms, as the Dutch GP guideline recommends an additional NG test in high-risk groups or when having symptoms. ${ }^{12}$ An NG retest is not mentioned in the Dutch GP guideline. ${ }^{12}$ Positivity of NG retesting was $0 \%$ in the current study, which may be explained by the low rate of retesting ( 15\%). Recently, another study among GPs in the Netherlands showed a retest positivity rate for NG of $\sim 23 \% .18$ 
As studies which include positivity in NG retesting of GP patients are scarce, more research is needed for NG control optimization. ${ }^{18}$

A CT TOC should never be performed within three weeks after CT treatment due to false-positive results what could lead to overtreatment. ${ }^{7-9}$ A CT TOC is in general not needed but should be performed (1) in the case of pregnancy, (2) when having persistent symptoms or (3) when there is lack of treatment with first choice treatment, as described in the Dutch GP guideline..2 An NG TOC is internationally recommended at least two weeks post treatment due to increasing antimicrobial resistance of NG. ${ }^{111}$ Unfortunately this is not recommended in the Dutch GP guideline..2 All CT and NG patients should be retested within three to twelve months, despite that the minimum time of three months is not mentioned in the Dutch GP guideline. ${ }^{12}$ Extragenital testing should be performed in all MSM and patients reporting anorectal intercourse or symptoms, as described in the Dutch GP guideline. ${ }^{12}$

To facilitate retesting at the GP practice, GPs could consider using modern testing and communication strategies such as e-health. For example, GPs could, in collaboration with their diagnostic laboratory and public health services, send home sampling kits 3 to 12 months post treatment to improve retesting rates. Also automatic small text messages could be used. These two methods have shown to increase retesting rates in specific settings, but not in GP settings yet. ${ }^{23} 33$ Moreover, education and awareness of the importance of CT and NG testing could improve (re)testing rates at the GP practice..$^{34}$

\section{Conclusion}

Almost 1 in every 5 CT positives returned for a TOC. Especially GP practices in lower SES areas performed a CT TOC, which is not recommended. Most patients did not have a CT retest, although the high CT positivity at retesting (12\%) demonstrates the need to encourage retesting of CT positives. The proportion of retesting for NG positives was also low (15\%). Moreover, only $0.3 \%$ of the $\mathrm{CT} / \mathrm{NG}$ consultations had patients tested on extragenital sites. As a result, infections or reinfections could have been missed.

A TOC for CT is not needed, whereas a TOC for NG should be performed. All CT and NG patients should be retested within three to twelve months. GPs should perform extra genital testing in all MSM and in all patients with anorectal intercourse or symptoms. The Dutch GP guideline needs to be reconsidered, especially regarding NG TOC and retesting as it is inconsistent with international guidelines. 


\section{References}

1. Newman L, Rowley J, Vander Hoorn S, et al. Global Estimates of the Prevalence and Incidence of Four Curable Sexually Transmitted Infections in 2012 Based on Systematic Review and Global Reporting. PLoS One 2015;10(12):e0143304. doi: 10.1371/journal.pone.0143304.

2. den Heijer CD, van Liere GA, Hoebe CJ, et al. Who tests whom? A comprehensive overview of Chlamydia trachomatis test practices in a Dutch region among different STI care providers for urogenital, anorectal and oropharyngeal sites in young people: a cross-sectional study. Sex Transm Infect 2016;92(3):211-7. doi: 10.1136/sextrans-2015-052065.

3. van den Broek IV, Verheij RA, van Dijk CE, et al. Trends in sexually transmitted infections in the Netherlands, combining surveillance data from general practices and sexually transmitted infection centers. BMC Fam Pract 2010;11:39. doi: 10.1186/1471-2296-11-39.

4. Santella AJ, Pollack A, Harrison C, et al. Management rates of sexually transmissible infections by Australian general practitioners, 2000-2012. Sex Health 2014;11(1):52-7. doi: 10.1071/SH13179.

5. Wetten S, Mohammed H, Yung M, et al. Diagnosis and treatment of chlamydia and gonorrhoea in general practice in England 2000-2011: a population-based study using data from the UK Clinical Practice Research Datalink. BMJ Open 2015;5(5):e007776. doi: 10.1136/bmjopen-2015-007776.

6. Shaw K, Stephens N, Coleman D, etal. Role of the general practitioner in testing for genital Chlamydia trachomatis infection: an analysis of enhanced surveillance data. Sex Health 2009;6(3):208-12. doi: 10.1071/SH09010.

7. Lanjouw E, Ouburg S, de Vries HJ, et al. 2015 European guideline on the management of Chlamydia trachomatis infections. International journal of STD \& AIDS 2016;27(5):333-48. doi: 10.1177/0956462415618837.

8. Nwokolo NC, Dragovic B, Patel S, et al. 2015 UK national guideline for the management of infection with Chlamydia trachomatis. International journal of STD \& AIDS 2016;27(4):251-67. doi: 10.1177/0956462415615443.

9. Workowski KA. Centers for Disease Control and Prevention Sexually Transmitted Diseases Treatment Guidelines. Clin Infect Dis 2015;61 Suppl 8:S759-62. doi: 10.1093/cid/civ771.

10. Lazaro N. Sexually Transmitted Infections in Primary Care 2013 (RCGP/BASHH) available at www. rcgp.org and www.bashh.org/guidelines [cited 201720 April]. accessed 4-11-2019.

11. Bignell C, Unemo M, European STIGEB. 2012 European guideline on the diagnosis and treatment of gonorrhoea in adults. International journal of STD \& AIDS 2013;24(2):85-92. doi: 10.1177/0956462412472837.

12. Van Bergen JEAM, et al. Standard of the National Society for General Practice: the STI consultation [NHGStandaard: Het soa-consult]. Huisarts en Wetenschap 2013;56(9):450-63.

13. Hosenfeld CB, Workowski KA, Berman S, et al. Repeat infection with Chlamydia and gonorrhea among females: a systematic review of the literature. Sex Transm Dis 2009;36(8):478-89. doi: 10.1097/OLQ.0b013e3181a2a933. 
14. Fung M, Scott KC, Kent CK, et al. Chlamydial and gonococcal reinfection among men: a systematic review of data to evaluate the need for retesting. Sex Transm Infect 2007;83(4):304-9. doi: 10.1136/ sti.2006.024059.

15. Xiridou M, Soetens LC, Koedijk FD, et al. Public health measures to control the spread of antimicrobial resistance in Neisseria gonorrhoeae in men who have sex with men. Epidemiol Infect 2015;143(8):1575-84. doi: 10.1017/S0950268814002519.

16. Bowring AL, Gouillou M, Guy R, et al. Missed opportunities--low levels of chlamydia retesting at Australian general practices, 2008-2009. Sex Transm Infect 2012;88(5):330-4. doi: 10.1136/ sextrans-2011-050422.

17. Dukers-Muijrers NH, van Liere GA, Hoebe CJ. Re-screening Chlamydia trachomatis positive subjects: a comparison of practices between an STI clinic, general practitioners and gynaecologists. Sex Transm Infect 2013;89(1):25-7. doi: 10.1136/sextrans-2011-050467.

18. den Heijer CDJ, Hoebe C, van Liere G, et al. A comprehensive overview of urogenital, anorectal and oropharyngeal Neisseria gonorrhoeae testing and diagnoses among different STI care providers: a cross-sectional study. BMC Infect Dis 2017;17(1):290. doi: 10.1186/s12879-017-2402-0.

19. Chan PA, RobinetteA, Montgomery M, etal. Extragenital Infections Caused by Chlamydia trachomatis and Neisseria gonorrhoeae: A Review of the Literature. Infect Dis Obstet Gynecol 2016;2016:5758387. doi: 10.1155/2016/5758387.

20. Dukers-Muijrers NHTM, Schachter J, van Liere GAFS, et al. What is needed to guide testing for anorectal and pharyngeal Chlamydia trachomatis and Neisseria gonorrhoeae in women and men? Evidence and opinion. BMC Infectious Diseases 2015;15:533. doi: 10.1186/s12879-015-1280-6.

21. Trienekens SC, van den Broek IV, Donker GA, et al. Consultations for sexually transmitted infections in the general practice in the Netherlands: an opportunity to improve STI/HIV testing. BMJ Open 2013;3(12):e003687. doi: 10.1136/bmjopen-2013-003687.

22. Kauhl B, Heil J, Hoebe CJ, et al. The Spatial Distribution of Hepatitis C Virus Infections and Associated Determinants--An Application of a Geographically Weighted Poisson Regression for Evidence-Based Screening Interventions in Hotspots. PLoS One 2015;10(9):e0135656. doi: 10.1371/ journal.pone.0135656.

23. Kampman C, Koedijk F, Driessen-Hulshof H, et al. Retesting young STI clinic visitors with urogenital Chlamydia trachomatis infection in the Netherlands; response to a text message reminder and reinfection rates: a prospective study with historical controls. Sex Transm Infect 2016;92(2):124-9. doi: 10.1136/sextrans-2015-052115.

24. Hughes $G$, Nichols T, Peters $L$, et al. Repeat infection with gonorrhoea in Sheffield, UK: predictable and preventable? Sex Transm Infect 2013;89(1):38-44. doi: 10.1136/sextrans-2012-050495.

25. Downing SG, Cashman C, McNamee H, et al. Increasing chlamydia test of re-infection rates using SMS reminders and incentives. Sex Transm Infect 2013;89(1):16-9. doi: 10.1136/sextrans-2011-050454.

26. Guy R, Wand H, Knight V, et al. SMS reminders improve re-screening in women and heterosexual men with chlamydia infection at Sydney Sexual Health Centre: a before-and-after study. Sex Transm Infect 2013;89(1):11-5. doi: 10.1136/sextrans-2011-050370. 
27. Gotz HM, Wolfers ME, Luijendijk A, et al. Retesting for genital Chlamydia trachomatis among visitors of a sexually transmitted infections clinic: randomized intervention trial of home- versus clinicbased recall. BMC Infect Dis 2013;13:239. doi: 10.1186/1471-2334-13-239.

28. Barbee LA, Golden MR. Editorial Commentary: When to Perform a Test of Cure for Gonorrhea: Controversies and Evolving Data. Clin Infect Dis 2016;62(11):1356-9. doi: 10.1093/cid/ciw142 [published Online First: 2016/03/11].

29. Beymer MR, Llata E, Stirland AM, et al. Evaluation of gonorrhea test of cure at 1 week in a Los Angeles community-based clinic serving men who have sex with men. Sex Transm Dis 2014;41(10):595-600. doi: 10.1097/OLQ.0000000000000190.

30. Dukers-Muijrers NH, Morre SA, Speksnijder A, et al. Chlamydia trachomatis test-of-cure cannot be based on a single highly sensitive laboratory test taken at least 3 weeks after treatment. PLoS One 2012;7(3):e34108. doi: 10.1371/journal.pone.0034108.

31. NethMap-MARAN 2016; Monitoring of Antimicrobial Resistance and Antibiotic Usage in Animals in the Netherlands in 2014; Nethmap: Consumption of antimicrobial agents and antimicrobial resistance among medically important bacteria in the Netherlands [Available from: http:// www.wur.nl/upload_mm/0/b/c/433ca2d5-c97f-4aa1-ad34-a45ad522df95_92416_008804_ NethmapMaran2016+TG2.pdf accessed 10 April 2017.

32. Rose SB, Garrett SM, Stanley J, et al. Retesting and repeat positivity following diagnosis of Chlamydia trachomatis and Neisseria gonorrhoea in New Zealand: a retrospective cohort study. BMC Infect Dis 2017;17(1):526. doi: 10.1186/s12879-017-2635-y.

33. Dukers-Muijrers NH, Theunissen KA, Wolffs PT, et al. Acceptance of Home-Based Chlamydia Genital and Anorectal Testing Using Short Message Service (SMS) in Previously Tested Young People and Their Social and Sexual Networks. PLoS One 2015;10(7):e0133575. doi: 10.1371/journal. pone.0133575.

34. Yeung A, Temple-Smith M, Spark S, et al. Improving chlamydia knowledge should lead to increased chlamydia testing among Australian general practitioners: a cross-sectional study of chlamydia testing uptake in general practice. BMC Infect Dis 2014;14:584. doi: 10.1186/s12879-014-0584-2. 


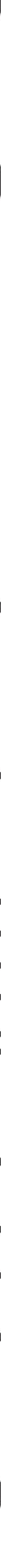





\section{Abstract}

\section{Background}

Repeat Chlamydia trachomatis (CT) infections are common. We assessed whether patients repeatedly infected with $\mathrm{CT}$ were different compared to patients repeatedly tested negative to obtain insight in the characteristics of patients frequently tested and infected with $\mathrm{CT}$ at our STI care services.

\section{Methods}

We assessed and included CT consultations between 2011 and mid-2018 from the laboratory covering one defined geographical area $(n=27,026)$. Multivariable logistic regression analyses were performed for the outcomes 'repeat CT infections', and 'once CT positive and not repeatedly tested'.

\section{Results}

Patients aged <25 years (OR: 1.83; 95\%Cl:1.38-2.43), co-infected with HIV (OR: 2.07; 95\%Cl: 1.02-4.22) or co-infected with Neisseria gonorrhoeae (NG) (OR: 5.04; 95\%Cl: 3.33-7.63) had more often had repeat CT infections. In additional analyses among only STI clinic visitors, patients with urogenital symptoms (OR: 2.17; 95\%Cl: 1.41-3.35), and patients notified for STIS (OR: 4.55; 95\%Cl: 3.17-6.54) had more often repeat CT infections. Men (OR: 2.63; 95\%Cl: 2.21 3.13), and patients aged <25 (OR: 2.92; 95\% Cl: 2.44-3.50) were more often CT positive and not repeatedly tested.

\section{Conclusions}

Patients aged $<25$ years, and patients coinfected with HIV or NG had more often repeat CT infections, accounted for $20 \%$ of the diagnosed CT infections, and are likely at highest risk for transmitting and acquiring CT. Nevertheless, $41 \%$ of the patients were not retested after diagnosis and thereby lost to care, indicating missed repeat $\mathrm{CT}$ infections and ongoing transmission. 


\section{Introduction}

Chlamydia trachomatis (CT) infection is one of the most commonly diagnosed bacterial sexually transmitted infections (STIS) worldwide. ${ }^{1}$ International testing guidelines advocate retesting within 3 to 12 months for all patients who test positive for CT. ${ }^{2-4}$ Repeat CT infections are common; up to $32 \%$ of CT patients test positive again within one year after diagnosis. ${ }^{5-7}$

Previous studies have indicated that patients with more sexual partners, women, younger patients, and patients coinfected with other STIS are more likely to have a repeat CT infection within 1 year of a previous CT infection. 8-12 These studies compared the characteristics of patients with 1 repeat infection to those patients with no repeat infection. To date, it is unknown whether the characteristics of patients with more than one repeat CT infection over several years differ from those patients repeatedly tested negative for CT. ${ }^{9}$ Differences in characteristics between patients repeatedly infected with CT and those repeatedly tested negative for CT could be indicative of different high risk populations. However, similarities in the characteristics between these groups could be indicative of similar sexual networks and high-risk behavior. ${ }^{1314}$ Furthermore, the extent to which patients with repeat CT infections account for the total number of diagnosed CT infections could provide insight in CT transmission routes. ${ }^{9}$

In contrast, patients who are not repeatedly tested after their initial diagnosis could also have impact on the circulating STIS within a population. For example, according to previous studies, CT retesting rates in STI clinics and general practitioners (GPS) are relatively low, ranging from $15 \%$ to $40 \%{ }^{71516}$, whereas reinfection rates are relatively high (12\%-18\%). ${ }^{71} 16$ Therefore, reinfections are likely missed, enabling ongoing transmission. It remains unclear whether the characteristics of patients who test positive and who are not repeatedly tested are different than those patients who repeatedly test negative, possibly indicating missed CT infections among high-risk individuals.

Here, we compared the socio-demographic characteristics of patients with repeat CT infections to patients who repeatedly test negative for CT. Furthermore, we assessed the socio-demographic characteristics of patients who were once tested CT positive and not repeatedly tested to obtain insight in the population lost to care. To achieve this, we performed this cross-sectional study including all CT consultations of different STI care providers in a defined geographical area.

\section{Methods}

\section{Study population}

From January 2011 to July 2018, all CT test consultations ( $n=27,026)$ of 17,639 patients aged between 15 and 64 years were obtained from the database of the regional Medical Microbiol- 
ogy Laboratory of the Maastricht University Medical Center (MUMC+) (Figure 1). The database comprised consultations from all STI care providers, including mental healthcare facilities ( $n=178 ; 0.7 \%)$, STI clinic ( $n=12,170 ; 45.0 \%)$, hospital ( $n=3,667 ; 13.6 \%)$, and GPs ( $n=11,011$; $40.7 \%$ ), from a geographical area in the south-eastern part of Limburg, the Netherlands. In the study area, $81 \%(n=48)$ GPs send the CT samples for testing to the regional laboratory, ensuring sufficient laboratory coverage. ${ }^{7}$ Samples tested positive for CT within 30 days of a previous positive CT test were excluded due to possible false-positive results ${ }^{4}(n=233)$; the vast majority of excluded tests (86.7\%; 202/233) were diagnosed within 14 days of a previous CT infection.

The geographical area consisted of 111,162 inhabitants (hereafter referred to as the "residential population") aged between 15 and 64 years (Statistics Netherlands: https://www. cbs.nl/en-gb). We calculated what proportion of the residential population was tested for $\mathrm{CT}$, once infected, and repeatedly infected with CT.

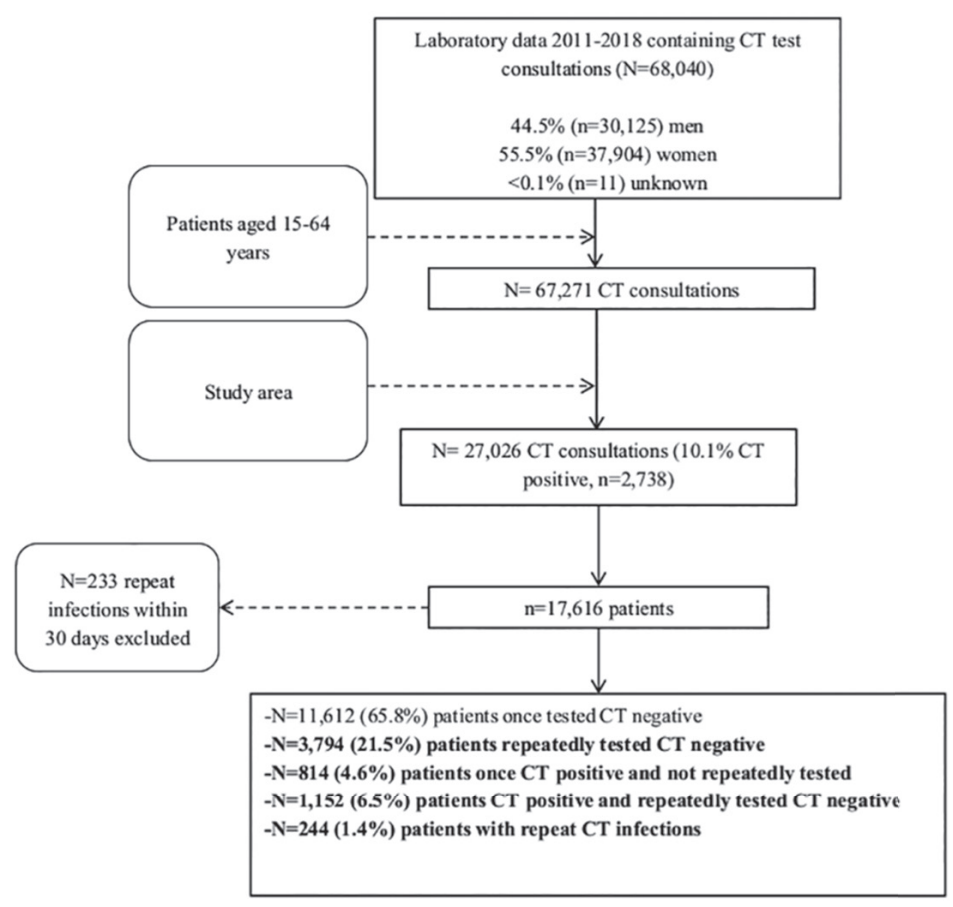

Figure 1. Flowchart of Chlamydia trachomatis test consultations, January 2011- July 2018

\section{Outcome measures}

Two main outcome measures were defined as: (1) "repeat CT infections," which included patients with $\geq 2$ repeat CT infections within the study period and (2) "Once CT positive and not 
repeatedly tested," which included patients who were once tested positive for CT and not repeatedly tested.

\section{Statistical analyses}

Our main study population included all individuals tested for CT ( $n=17,616)$. In our main analyses, we assessed whether the characteristics of individuals classified in one of the two above described outcome measures were different compared to individuals who repeatedly tested negative ( $\geq 2$ negative CT tests) using multivariable logistic regression analyses. The determinants that were tested were initial test location (mental healthcare facilities, STI clinic, hospital, or GPs), sex (men or women), age (<25 years and $\geq 25$ years), urbanization (rural or urban), HIV coinfection (not tested, yes, or no), and NG coinfection (not tested, yes, or no). All determinants were recorded at the time of the patient's consultation. We noticed that $6.2 \%$ ( $n=1,090)$ of the patients tested for CT changed their STI care provider after their initial test (Supplementary Table S1). Therefore, the determinant "initial test location" was based on the STI care provider where the patient was firstly tested.

Additional determinants were available for the STI clinic population only. Therefore, our secondary study population included only STI clinic visitors tested for CT ( $n=8,007$ ). Additional determinants included: the maximum number of sex partners in the past 6 months prior to a consultation a patient had in the entire study period (unknown, $0-1,2-3$, and $\geq 4$ ), any urogenital symptoms during the study period (unknown, yes, or no), any proctitis during the study period (unknown, yes, or no), any oropharyngeal symptoms during the study period (unknown, yes, or no), any notification for STI during the study period (unknown, yes, or no), and transmission group (men who have sex with women [MSW] or men who have sex with men [MSM], and women).

Determinants with $\mathrm{P}<0.10$ in the univariable logistic regression models were included in the multivariable model. Odds ratios and 95\% confidence intervals (Cls) were calculated and presented. All analyses were performed using the SPSS V24 (IBM SPSS Statistics for Windows, IBM Corporation, Armonk, New York, USA). A P-value of <0.05 was considered statistically significant.

\section{Ethics statement}

The Medical Ethics Committee of the Maastricht University Medical Center (Maastricht, the Netherlands) approved this study (METC 2017-0251) and waived the need for patient consent. Since the retrospective data originated from regular care and were analyzed anonymously, no further informed consent for data analysis was obtained. 


\section{Patient and public involvement}

The development of the research question and outcome measures were not informed by patients' priorities, experience, and preferences. No patients were involved in the design of this study. No patients were involved in the recruitment to and conduct of the study. The study results will not be disseminated to study participants since retrospective data were used.

\section{Results}

\section{$\mathrm{CT}$ testing and positivity in the residential population}

Of the 111,162 individuals residing in the study area, $15.8 \%(n=17,616)$ were tested for $C T$ and $2.0 \%(n=2,210)$ were tested positive at least once; $1.8 \%(n=1,966)$ were diagnosed with 1 CT infection, and $0.2 \%$ ( $n=244)$ with $\geq 2$ CT infections.

Within the individuals tested for $\mathrm{CT}(\mathrm{n}=17,616), 11.2 \%(\mathrm{n}=1,966)$ were tested positive for CT once, and $1.4 \%(n=244)$ were repeatedly $(\geq 2)$ infected with $C T$. The characteristics of all individuals tested for $\mathrm{CT}$ are presented in Table 1.

Of the 1,966 patients once infected with CT, $41.4 \%(n=814)$ were not repeatedly tested after their CT diagnosis. All 2,210 CT patients contributed to 2,505 CT infections. Of these 2,505 CT infections, repeat CT infections ( $\geq 2$ CT infections) accounted for $21.5 \%(n=539)$. 


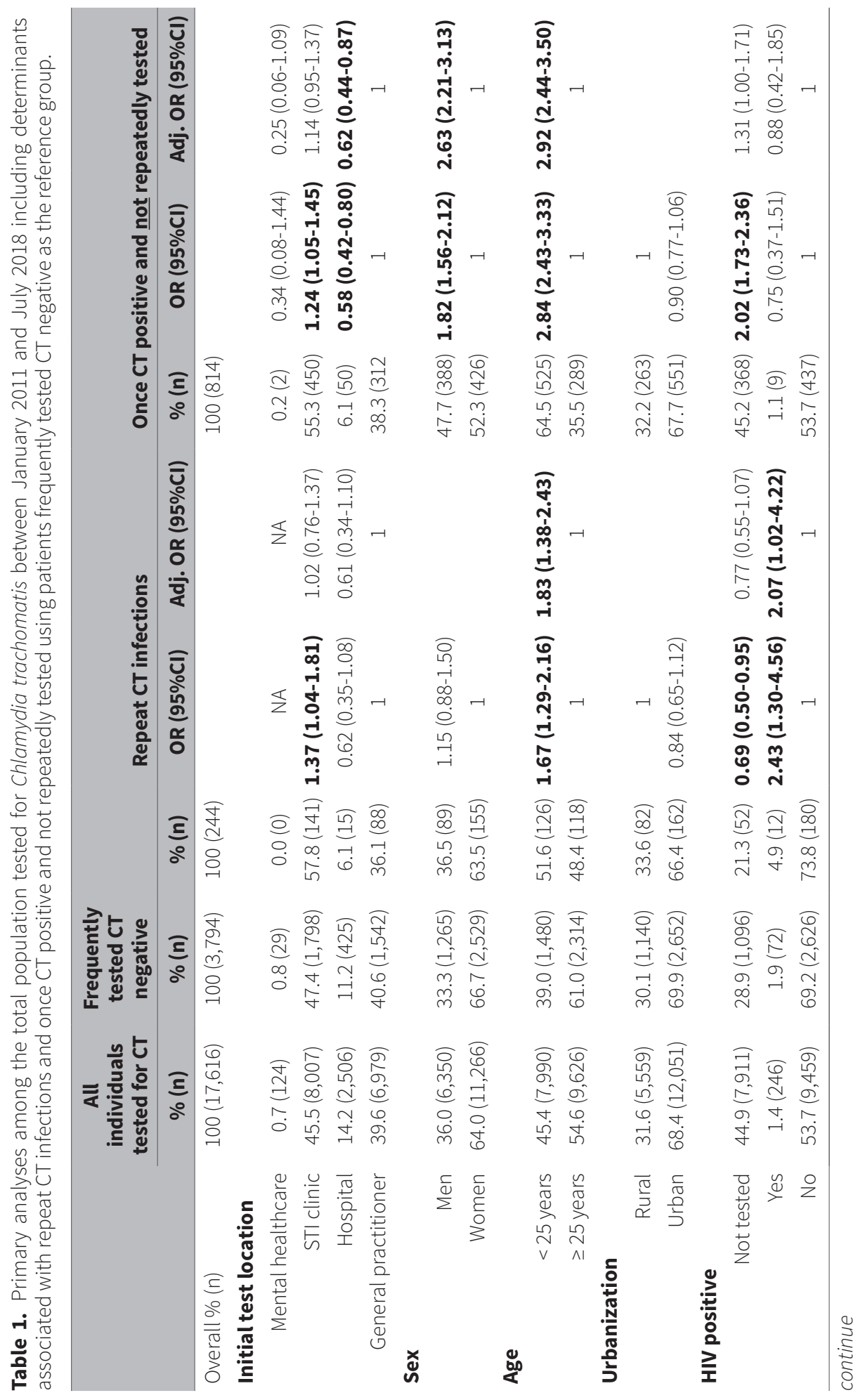




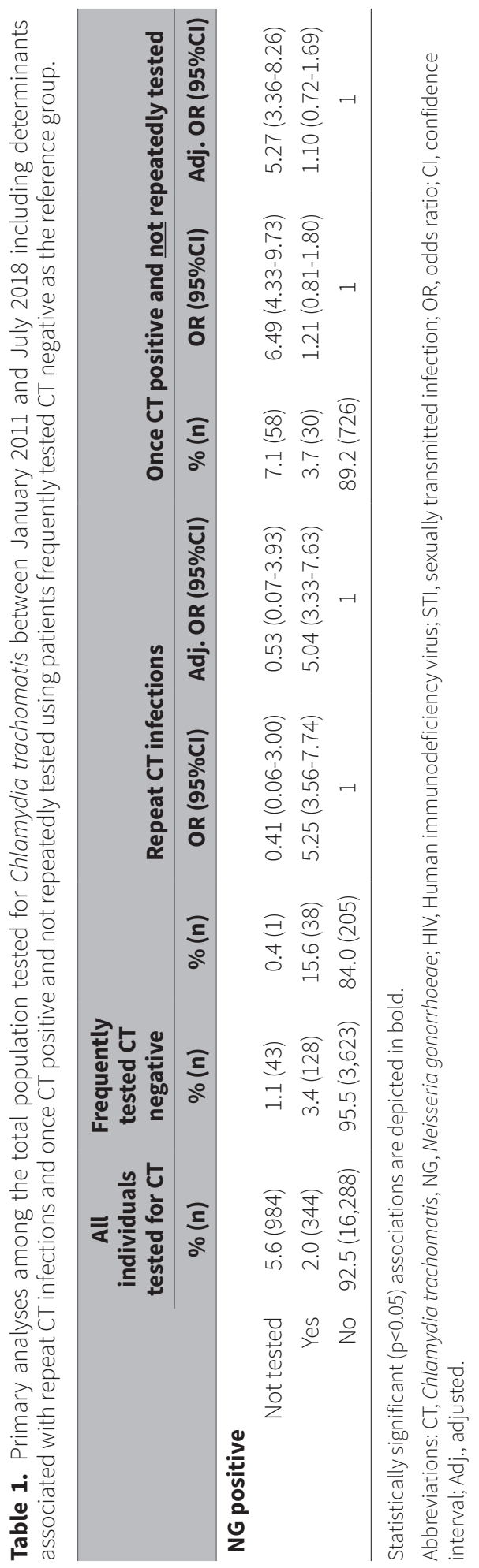




\section{Characteristics of patients with repeat infections}

Patients repeatedly infected with CT ( $\geq 2$ CT infections) accounted for $1.4 \%$ ( $n=244$ ) of all the 17,616 individuals tested for CT (Figure 1). In multivariable analyses, patients repeatedly infected with CT were more likely aged $<25$ years, coinfected with HIV, or coinfected with NG compared to patients repeatedly tested negative for CT (Table 1 ).

In our secondary analyses among only STI clinic visitors, patients repeatedly infected with CT ( $n=141$ ) were more likely to have urogenital symptoms or notified for STIs compared to patients repeatedly tested negative for CT (Table 2 ). 


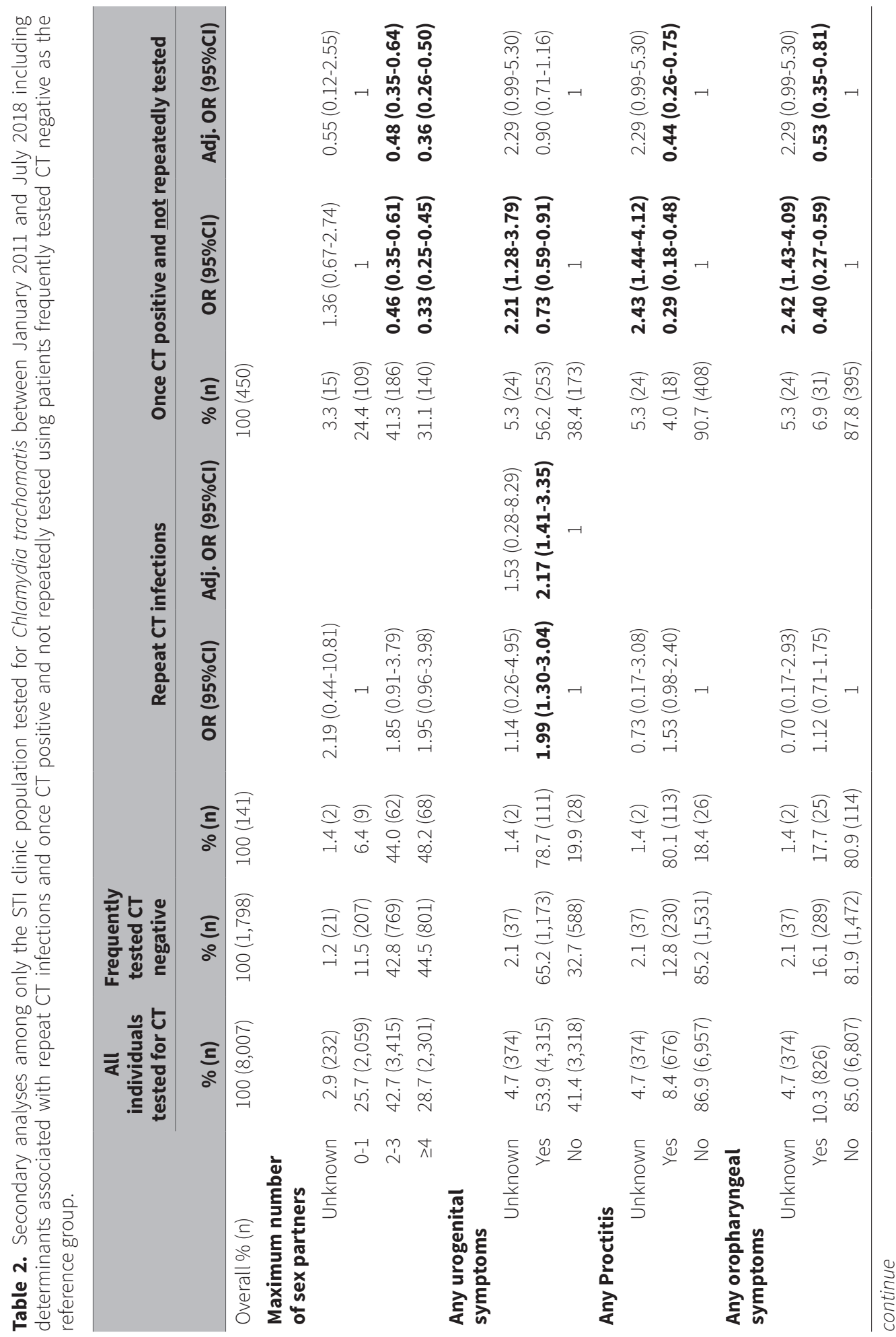




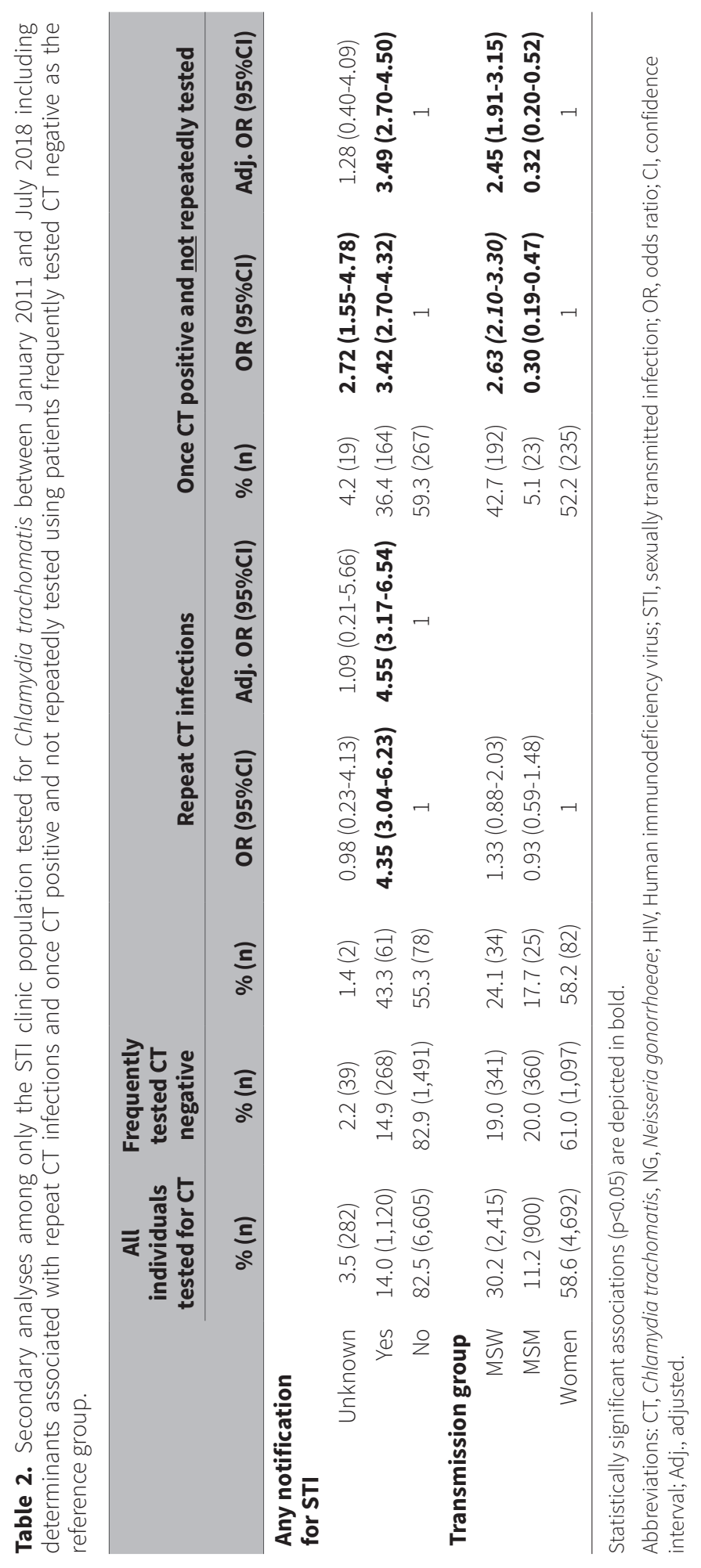




\section{Characteristics of patients who once tested positive for $\mathrm{CT}$ and not repeatedly tested}

Of all the 17,616 individuals tested for CT, 4.6\% ( $n=814)$ were found to be positive for CT once and not repeatedly tested (Figure 1 ). In multivariable analyses, these patients were more often men, aged < 25 years, and not tested for NG compared to patients repeatedly tested negative for CT (Table 1$)$.

In our secondary analyses among only STI clinic visitors, patients once tested positive for CT and not repeatedly tested ( $n=450)$ were more often notified for STIs or MSW compared to patients repeatedly tested negative for CT (Table 2).

\section{Discussion}

In this study, we included all CT consultations at STI care providers in a defined geographical area to assess whether patients repeatedly infected with $\mathrm{CT}$ were different to those repeatedly tested negative. Patients aged $<25$ years or coinfected with NG or HIV more often had repeat CT infections and are likely at highest risk for acquiring and transmitting CT. Those patients with repeat $\mathrm{CT}$ infections accounted for $22 \%$ of all diagnosed $\mathrm{CT}$ infections. Additional focus should be on men, patients aged < 25 years, and patients not tested for NG as they were more often tested positive for CT once without repeat testing, likely causing missed repeat CT infections and ongoing transmission.

A strength of our study is that all CT consultations of all STI care providers in a defined geographical area were included to obtain insight in CT testing differences between STI care providers. Due to the inclusion of all tests by the STI clinics and medical specialists and the high coverage of GP data (81\%), underestimations of CT tests seem unlikely. ${ }^{7}$ Another strength is the timeframe of 7.5 year to partly prevent underestimations of repeat infections. ${ }^{9}$ We excluded all CT tests which were positive within 30 days of an earlier positive test to be able to draw accurate conclusions about patients repeatedly infected, i.e., patients with a positive test within 30 days could be false-positive due to non-viable recurrent CT. ${ }^{4}$ The additional analyses among STI clinic visitors allowed us to assess additional determinants related to sexual behavior to draw conclusions specified for STI clinic visitors.

A limitation of the study was that information on reasons for testing was unavailable. Such reasons may include financial reasons. For example, STI tests at the GP are within patients' deductibles in healthcare insurances, whereas STI tests at the STI clinic are free of charge for risk groups (age <25 years, MSM, and commercial sex workers). ${ }^{17}{ }^{18}$ Such reasons for testing could provide more insight into whether patients were repeatedly tested. Furthermore, the additional determinants (sexual risk behavior and symptoms) assessed in the STI clinic population were not available for the mental healthcare, GP, and hospital population. We were unable to assess whether patients moved out of the study area or were repeat tested 
by another STI care provider out of the study area potentially leading to an underestimation of repeat infections or repeat tests. However, we expect this potential bias to be low, since repeat testing in general at different STI care providers, such as the STI clinic and GP, is proven to be low. ${ }^{71519}$ A general limitation of studies using existing databases is that data is only available for the tested population, and probably a proportion of all CT infections remain undiagnosed and are not considered. However, this study is one of the few to include all tests by all care providers in one geographical area, therefore including at least almost the whole tested population.

A comparable study by Hsu et al. showed that $28 \%$ of all diagnosed STIs were repeat $(\geq 2)$ infections. ${ }^{9}$ They concluded that a relatively small group of patients repeatedly infected with STIs likely have a disproportionally high impact on circulating STIs within a population; the so called "core group". ${ }^{9}$ The authors included all relevant STIs in their case definition including repeat syphilis, NG, and CT infections. However, we believe that the "core group" theory is STI specific. For example, 76\% of the NG infections are diagnosed among MSM in the Netherlands. Whereas CT infections are distributed among different transmission groups (23\% of the CT diagnoses are among MSM, 50\% among women, and 28\% among heterosexual men)..$^{20}$ Moreover, heterosexual men, women, and MSM were equally affected by repeat CT infections arguing for population transmission instead of core group transmission ( $p>0.05$; Table 2). Furthermore, 16\% of the residential population in our study area was tested for CT. Therefore, only a small fraction of all CT infections was possibly diagnosed, leading to ongoing transmission within the population.

Despite patients undergoing repeat testing and with repeat infections are at a high risk for transmitting and acquiring STIS, many CT patients (41\%) were once CT positive and not repeatedly tested and, therefore, lost to care. This enables ongoing transmission as repeat CT infections are common among patients undergoing retesting within 1 year (up to 32\%). ${ }^{715}$ ${ }^{16} 19$ Retesting CT-positive patients is an effective control strategy and can be used to enhance the population-based prevention of $\mathrm{CT}^{4}{ }^{4}$ Although retesting is advised in many international guidelines ${ }^{242122}$, retesting rates remain typically low. ${ }^{7151619}$ Focus should be on men and patients aged $<25$ years as they were more often tested positive for $\mathrm{CT}$ and not retested, probably leading to ongoing transmission of $\mathrm{CT}$.

NG coinfection was associated with repeat CT infections. Notably, 58 patients who were once CT positive and not repeatedly tested were not tested for NG (Table 1). We noticed that almost all these patients were from the GP and hospital population (94.8\% 55/58). Therefore, NG infections and CT repeat infections could be missed, leading to ongoing transmission of CT and NG. GPs and hospital physicians should consider CT and NG testing at the same consultation for all patients at a risk for STIs. ${ }^{42324} \mathrm{CT}$ and NG are tested using the same sample, leading to no additional costs when assessing NG positivity.

STI clinic patients with urogenital symptoms were more likely to have repeat CT infections compared to patients without these symptoms. Our study group and others observed an association between urogenital symptoms and a higher CT bacterial load, which could be in- 
dicative of a higher transmission potential and likely clinical relevance. ${ }^{25-27}$ Furthermore, the highest CT positivity rates are found among patients notified for CT (35.2\% in women, 32.8\% in heterosexual men, and 22.5\% in MSM). ${ }^{20}$ Notably, STI clinic patients notified for STIS were more often repeatedly infected with $\mathrm{CT}$, indicating the essential role of partner management for targeting, testing, and treating this high-risk population. ${ }^{28}$

In conclusion, patients aged $<25$ years, and patients coinfected with HIV or NG had more often repeat CT infections accounting for one out of five diagnosed CT infections. Also, STI clinic patients with urogenital symptoms and notified for STI's were more often repeatedly infected with CT. Those patients are likely at highest risk for transmitting and acquiring CT. Nevertheless, $41 \%$ of the patients, mainly men, individuals aged $<25$ years and individuals not tested for NG, were not retested after diagnosis and thereby lost to care, indicating missed repeat $\mathrm{CT}$ infections and ongoing transmission. 


\section{References}

1. Newman L, Rowley J, Vander Hoorn S, et al. Global Estimates of the Prevalence and Incidence of Four Curable Sexually Transmitted Infections in 2012 Based on Systematic Review and Global Reporting. PLoS One 2015;10(12):e0143304. doi: 10.1371/journal.pone.0143304.

2. Bignell C, Unemo M, European STIGEB. 2012 European guideline on the diagnosis and treatment of gonorrhoea in adults. International journal of STD \& AIDS 2013;24(2):85-92. doi: $10.1177 / 0956462412472837$.

3. Lazaro N. Sexually Transmitted Infections in Primary Care 2013 (RCGP/BASHH) available at www. rcgp.org and www.bashh.org/guidelines [cited 201720 April].

4. Workowski KA. Centers for Disease Control and Prevention Sexually Transmitted Diseases Treatment Guidelines. Clin Infect Dis 2015;61 Suppl 8:S759-62. doi: 10.1093/cid/civ771.

5. Hosenfeld CB, Workowski KA, Berman S, et al. Repeat infection with Chlamydia and gonorrhea among females: a systematic review of the literature. Sex Transm Dis 2009;36(8):478-89. doi: 10.1097/OLQ.0b013e3181a2a933.

6. Fung M, Scott KC, Kent CK, et al. Chlamydial and gonococcal reinfection among men: a systematic review of data to evaluate the need for retesting. Sex Transm Infect 2007;83(4):304-9. doi: 10.1136/ sti.2006.024059.

7. Wijers J, van Liere G, Hoebe C, et al. Test of cure, retesting and extragenital testing practices for Chlamydia trachomatis and Neisseria gonorrhoeae among general practitioners in different socioeconomicstatus areas: Aretrospective cohortstudy, 2011-2016. PLoS One 2018;13(3):e0194351. doi: 10.1371/journal.pone.0194351.

8. Hughes G, Brady AR, Catchpole MA, et al. Characteristics of those who repeatedly acquire sexually transmitted infections: a retrospective cohort study of attendees at three urban sexually transmitted disease clinics in England. Sex Transm Dis 2001;28(7):379-86. [published Online First: 2001/07/19].

9. Hsu KK, Molotnikov LE, Roosevelt KA, et al. Characteristics of Cases With Repeated Sexually Transmitted Infections, Massachusetts, 2014-2016. Clin Infect Dis 2018;67(1):99-104. doi: 10.1093/ cid/ciy029.

10. Kinghorn GR, Pryce D, Morton RS. Repeated gonorrhea in Sheffield: the size of the problem, epidemiologic significance, and personal characteristics of repeaters. Sex Transm Dis 1982;9(4):165-9.

11. Visser M, van Aar F, Koedijk FDH, et al. Repeat Chlamydia trachomatis testing among heterosexual STI outpatient clinic visitors in the Netherlands: a longitudinal study. BMC Infect Dis 2017;17(1):782. doi: 10.1186/s12879-017-2871-1 [published Online First: 2017/12/22].

12. Bjekic M, Vlajinac H, Marinkovic J. Behavioural and social characteristics of subjects with repeated sexually transmitted diseases. Acta Derm Venereol 2000;80(1):44-7.

13. Gesink DC, Sullivan AB, Miller WC, et al. Sexually transmitted disease core theory: roles of person, place, and time. Am J Epidemio/ 2011;174(1):81-9. doi: 10.1093/aje/kwr035. 
14. Gotz HM, van Oeffelen LA, Hoebe C, et al. Regional differences in chlamydia and gonorrhoeae positivity rate among heterosexual STI clinic visitors in the Netherlands: contribution of client and regional characteristics as assessed by cross-sectional surveillance data. BMJ Open 2019;9(1):e022793. doi: 10.1136/bmjopen-2018-022793.

15. Bowring AL, Gouillou M, Guy R, et al. Missed opportunities--low levels of chlamydia retesting at Australian general practices, 2008-2009. Sex Transm Infect 2012;88(5):330-4. doi: 10.1136/ sextrans-2011-050422.

16. Rose SB, Garrett SM, Stanley J, et al. Retesting and repeat positivity following diagnosis of Chlamydia trachomatis and Neisseria gonorrhoea in New Zealand: a retrospective cohort study. BMC Infect Dis 2017;17(1):526. doi: 10.1186/s12879-017-2635-y.

17. Kampman C, Koedijk F, Driessen-Hulshof H, et al. Retesting young STI clinic visitors with urogenital Chlamydia trachomatis infection in the Netherlands; response to a text message reminder and reinfection rates: a prospective study with historical controls. Sex Transm Infect 2016;92(2):124-9. doi: 10.1136/sextrans-2015-052115.

18. Gotz HM, Wolfers ME, Luijendijk A, et al. Retesting for genital Chlamydia trachomatis among visitors of a sexually transmitted infections clinic: randomized intervention trial of home- versus clinicbased recall. BMC Infect Dis 2013;13:239. doi: 10.1186/1471-2334-13-239.

19. Dukers-Muijrers NH, van Liere GA, Hoebe CJ. Re-screening Chlamydia trachomatis positive subjects: a comparison of practices between an STI clinic, general practitioners and gynaecologists. Sex Transm Infect 2013;89(1):25-7. doi: 10.1136/sextrans-2011-050467.

20. Slurink I, van Aar F, Op de Coul E, et al. Sexually transmitted infections in the Netherlands in 2018: Rijksinstituut voor Volksgezondheid en Milieu.

21. Lanjouw E, Ouburg S, de Vries HJ, et al. 2015 European guideline on the management of Chlamydia trachomatis infections. International journal of STD \& AIDS 2016;27(5):333-48. doi: 10.1177/0956462415618837.

22. Nwokolo NC, Dragovic B, Patel S, et al. 2015 UK national guideline for the management of infection with Chlamydia trachomatis. International journal of STD \& AIDS 2016;27(4):251-67. doi: 10.1177/0956462415615443.

23. Van Bergen JEAM, et al. Standard of the National Society for General Practice: the STI consultation [NHGStandaard: Het soa-consult]. Huisarts en Wetenschap 2013;56(9):450-63.

24. De Vries H, van Dam A, Bax C. Seksueel Overdraagbare Aandoeningen Multidisciplinaire Richtlijn Update 20182018 [Available from: www.soaaids.nl/nl/professionals/beroepsgroep/arts/ professioneel-handelen/de-specialist accessed 3-9-2018.

25. Vodstrcil LA, Mclver R, Huston WM, et al. The Epidemiology of Chlamydia trachomatis Organism Load During Genital Infection: A Systematic Review. J Infect Dis 2015;211(10):1628-45. doi: 10.1093/ infdis/jiu670.

26. Wijers J, Hoebe C, van Liere G, et al. Chlamydia trachomatis bacterial load, estimated by Cq values, in urogenital samples from men and women visiting the general practice, hospital or STI clinic. PLoS One 2019;14(4):e0215606. doi: 10.1371/journal.pone.0215606. 
27. Jalal H, Verlander NQ, Kumar N, et al. Genital chlamydial infection: association between clinical features, organism genotype and load. J Med Microbiol 2011;60(Pt 7):881-8. doi: 10.1099/ jmm.0.028076-0.

28. Theunissen KA, Schipper P, Hoebe CJ, et al. Barriers to and facilitators of partner notification for chlamydia trachomatis among health care professionals. BMC Health Serv Res 2014;14:647. doi: 10.1186/s12913-014-0647-5. 


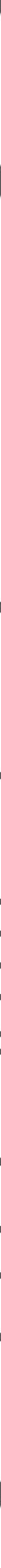





\section{Abstract}

We assessed whether patients repeatedly infected with Neisseria gonorrhoeae (NG) were different compared to patients repeatedly tested negative, to obtain insight into the characteristics of patients frequently tested and infected with NG. All patients tested for NG $(n=16,662)$ between January 2011 and July 2018 were included. Multivariable logistic regression analyses were performed for the outcomes "repeat NG infections" and "once NG positive and not retested" versus patients "repeatedly tested NG negative". Of the individuals tested for NG, $0.2 \%(40 / 16,662)$ had repeat $(\geq 2)$ NG infections, and accounted for $23 \%$ of all diagnosed NG infections. STI clinic patients, men (mostly men who have sex with men (MSM)), patients aged $\geq 25$ years, and patients co-infected with HIV or Chlamydia trachomatis (CT) more often had repeat NG infections. The number of patients not retested after their initial NG diagnosis was 29.9\% (92/308). Men (mostly MSM), HIV positive patients, and patients notified for sexually transmitted infections (STIS) were more often NG positive and not retested. Concluding, only 40 patients tested for NG accounted for one in four diagnosed NG infections. However, re-infections are likely to be missed among MSM and HIV positive patients, as they were mainly not retested after NG infection. It remains important to test and re-test for NG, especially in MSM, in order to halt transmission. 


\section{Introduction}

Neisseria gonorrhoeae (NG) is one of the most commonly diagnosed bacterial sexually transmitted infection (STI) worldwide. ${ }^{1}$ International testing guidelines advocate retesting within 3 to 12 months for all patients who test positive for NG.2-4 Repeat NG infections are common; up to $40 \%$ of NG patients test positive again within one year after diagnosis..$^{5-7}$

Previous studies have indicated that patients with more sexual partners, men who have sex with men (MSM), and patients co-infected with other STIs are more likely to have a repeat NG infection within one year of a previous NG infection. These studies compared the characteristics of patients with one repeat infection to those patients with no repeat infection to identify high risk individuals. To date, however, it remains unknown whether the characteristics of patients with more than one repeat NG infection differ from patients repeatedly tested NG negative. ${ }^{8}$ Differences in the characteristics between patients repeatedly infected with NG and those individuals repeatedly tested negative for NG could be indicative for different highrisk populations. However, similarities in the characteristics between those groups could suggest similar sexual networks and high risk behavior. 9,10 Furthermore, the extent to which patients with repeat NG infections account for the total number of diagnosed NG infections could provide insight into NG transmission routes. ${ }^{8}$

In contrast, patients who are not retested after their initial NG diagnosis could also have impact on circulating STIs within a population. For example, from previous studies it is known that NG retesting rates of STI clinics and general practitioners (GPS) are relatively low, ranging from 15-22.8\%, while reinfection rates are relatively high (up to 16\%). ${ }^{7,11}$ Therefore, NG reinfections are likely to be missed leading to ongoing transmission of NG. Identifying the characteristics of patients who are once NG positive and not retested could be used to inform NG (re-)test practices and control.

Here, we compared the socio-demographic characteristics of patients with repeat NG infections with individuals repeatedly tested NG negative to identify high risk populations. Furthermore, we assessed whether the socio-demographic characteristics of patients who were once NG positive and not retested were different compared to individuals repeatedly tested NG negative to obtain insight into the population lost to care. To achieve this, we performed this study including all NG consultations in a defined geographical area in a 7.5-year timeframe 


\section{Materials and methods}

\section{Study population}

In this cross-sectional study, all NG test consultations $(n=25,189)$ from January 2011 and July 2018 of 16,662 patients between 15 and 64 years old were obtained from the database of the regional Medical Microbiology Laboratory of Maastricht University Medical Center (MUMC+) (Figure 1). The database comprised consultations from all STI care providers from one geographical area in the south-eastern part of Limburg, the Netherlands. The study area included the municipalities of Maastricht, Eijsden-Margraten, and Valkenburg aan de Geul. Only data of patients living in this area were included for analyses. The distribution of NG test consultations per STI care provider were as follows: mental health care ( $n=171 ; 0.7 \%)$, the STI clinic ( $n=12,278 ; 48.7 \%$ ), the hospital ( $n=3206 ; 12.7 \%)$, and GPs ( $n=9534 ; 37.8 \%)$. Of the GP practices in the study area, $81 \%(n=48)$ send their tests to the regional laboratory ensuring acceptable laboratory coverage. ${ }^{7}$ NG positive tests within 30 days of a previous positive NG test were excluded due to possible false-positive results $(n=43){ }^{4}$

The geographical area consisted of $n=111,162$ inhabitants (hereafter residential population) between 15 and 64 years old. ${ }^{12}$ Our main study population included all individuals tested for NG $(n=16,662)$.

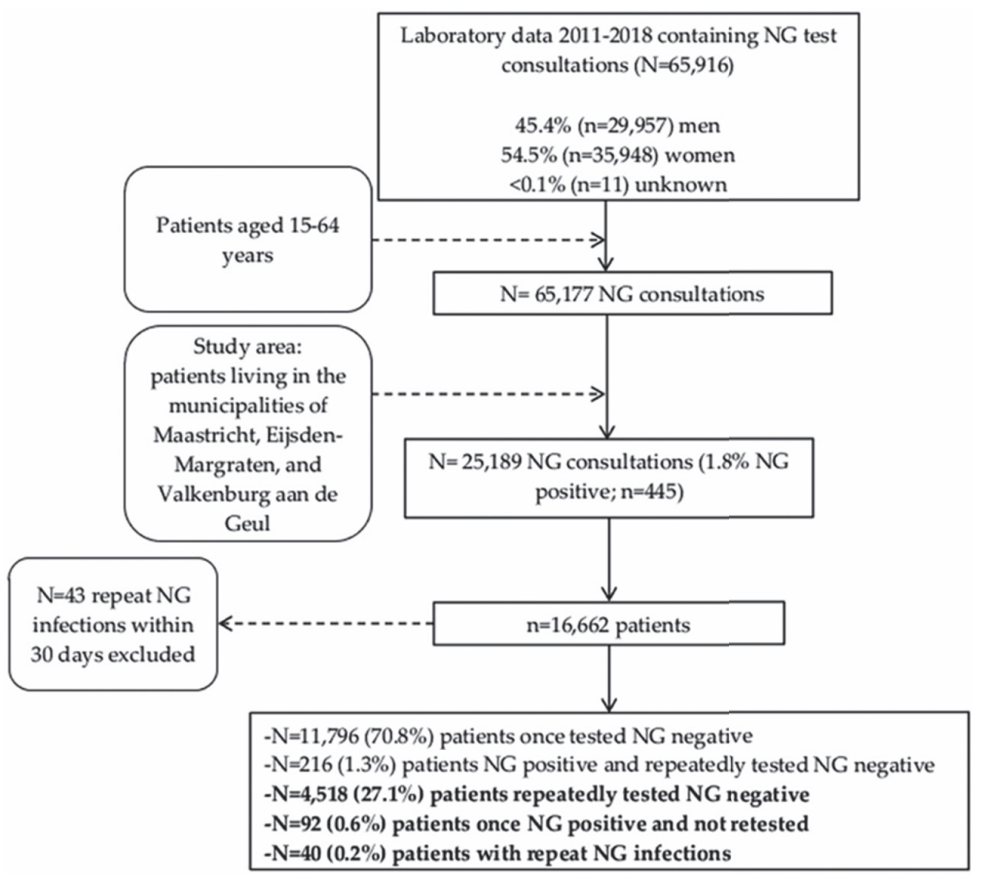

Figure 1. Flowchart of Neisseria gonorrhoeae test consultations, January 2011- July 2018. 


\section{Outcome measures}

Two main outcome measures were defined: (1) "repeat NG infections", which were patients with $\geq 2$ repeat NG infections within the study period, and (2) "once NG positive and not retested", which were patients who were once NG positive and not retested within the study period.

\section{Statistical analyses}

In our main analyses, we assessed whether the characteristics of patients classified in one of the two above described outcome measures were different compared to individuals repeatedly tested negative ( $\geq 2$ negative NG tests) using multivariable logistic regression analyses. Determinants tested were: initial test location (mental health care facilities, STI clinic, hospital, GP), sex (men, women), age (<25 years, $\geq 25$ years), urbanization (rural, urban), HIV co-infection (not tested, yes, no), and any CT co-infection (yes, no) during the study period. We noticed that 5.9\% ( $n=977$ ) of the patients tested for NG changed STI care provider after their initial test (Supplementary File 1). Therefore, the determinant 'initial test location' was based on the STI care provider where the patient was firstly tested for NG. The determinant urbanization was defined according to Statistics Netherlands: areas with $\geq 1500$ addresses per $\mathrm{km}^{2}$ were categorized as "urban" and areas with $<1500$ addresses per km² were categorized as "rural".12

Our secondary study population included STI clinic visitors tested for NG ( $n=8022$ ). Analyses were performed for determinants only available for the STI clinic population and included: the maximum number of sex partners in the past 6 months prior to a consultation of a patient in the entire study period (unknown, $0-1,2-3, \geq 4$ ), any urogenital symptoms during the study period (unknown, yes, no), any proctitis during the study period (unknown, yes, no), any oropharyngeal symptoms during the study period (unknown, yes, no), any notification for STI during the study period (unknown, yes, no), and transmission group (men who have sex with women (MSW), MSM and women).

We calculated which proportion of the residential population $(n=111,162)$ were tested for NG, once infected, and repeatedly infected with NG.

Determinants with $p<0.10$ in the univariable logistic regression models were included in the multivariable model. Odds ratios and 95\% confidence intervals $(\mathrm{Cl})$ were calculated and presented. All analyses were performed using SPSS V24 (IBM SPSS Statistics for Windows, IBM Corporation, Armonk, New York, USA). A p-value of < 0.05 was considered statistically significant.

Additionally, we visualized residence areas of patients (based on their 4-digit postal code), who were classified in one of the two earlier mentioned outcome measures, in a geographical map to inform potential outreach activities, and targeted testing using Qgis 2.18.28. ${ }^{13}$ 


\section{Ethics statement}

The medical ethics committee of the Maastricht University Medical Center (Maastricht, the Netherlands) approved this study (METC 2017-0251) and waived the need for consent to be collected from participants. Since retrospective data originated from regular care and were analyzed anonymously, no further informed consent for data analysis was obtained.

\section{Results}

\section{NG testing and positivity in the residential population}

Of the 111,162 people residing in the study area, $15.0 \%(n=16,662)$ were tested for NG. $0.3 \%$ $(n=348)$ tested positive at least once; $0.3 \%(n=308)$ were diagnosed with one NG infection; and $0.04 \%(n=40)$ with two or more NG infections.

Within the individuals tested for NG ( $n=16,662)$, the vast majority $(70.8 \%)$ were tested once and found NG negative $(n=11,796)$, and $0.2 \%(n=40)$ were repeatedly $(\geq 2)$ infected with NG (Figure 1). The characteristics of all individuals tested for NG are presented in Table 1.

Of the 308 patients once infected with NG, 29.9\% ( $n=92)$ were not retested after their initial NG diagnosis. All 348 NG positive patients contributed to 402 NG infections. Of these 402 NG infections, repeat NG infections ( $\geq 2$ NG infections) accounted for $23.4 \%(n=94)$. 


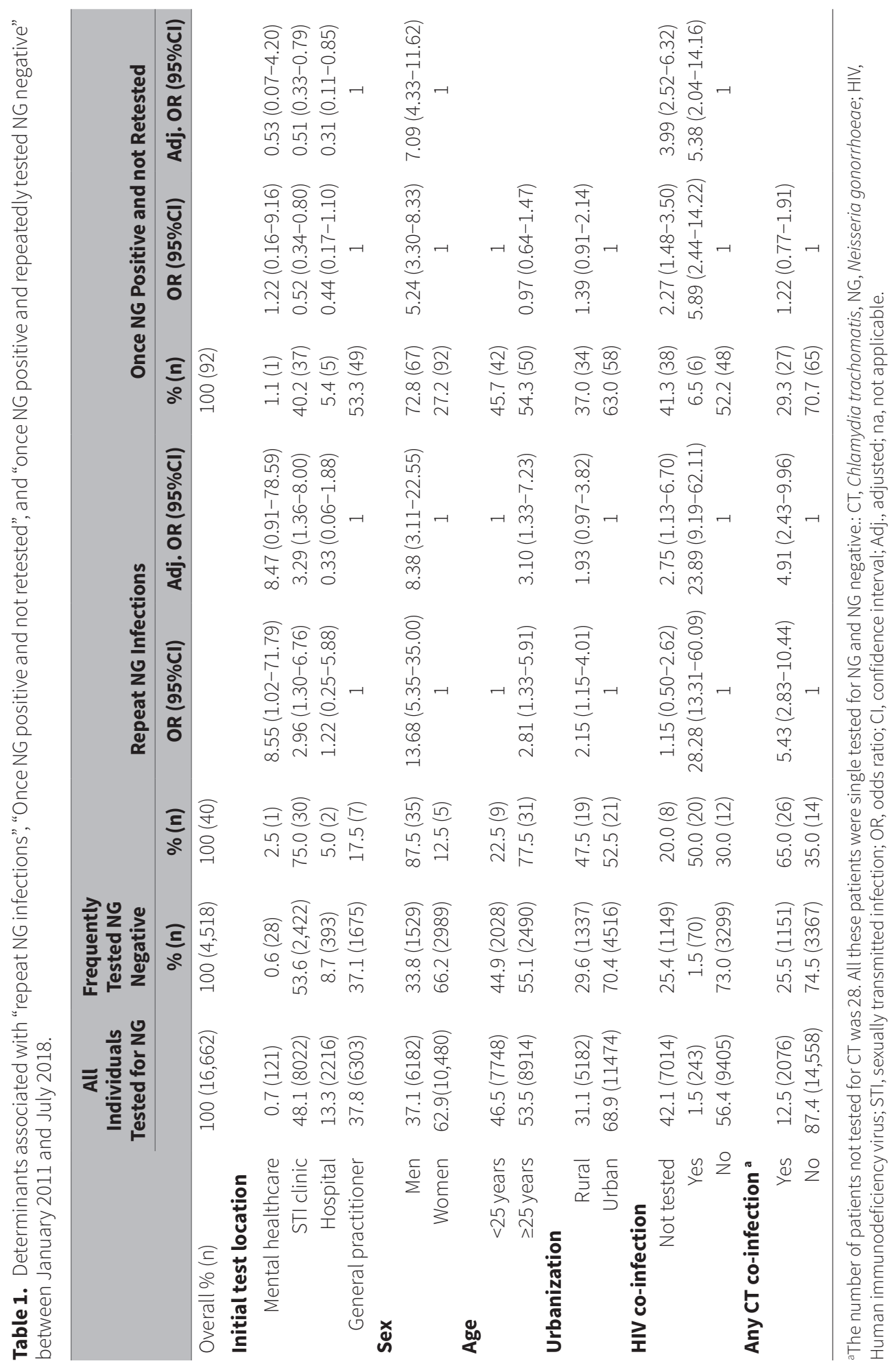




\section{Characteristics of patients with repeat NG infections}

The characteristics of patients repeatedly infected with NG ( $\geq 2$ NG infections) ( $n=40)$ were compared to the characteristics of patients repeatedly tested negative for NG $(n=4518)$. In multivariable analyses, patients repeatedly infected with NG were more likely STI clinic patients, men, aged $\geq 25$ years, not tested for HIV, co-infected with HIV, or co-infected with CT.

In our secondary analyses among STI clinic visitors, patients repeatedly infected with NG $(n=30)$ were more likely having urogenital symptoms, having proctitis, notified for STIs or MSM (Table 2). 


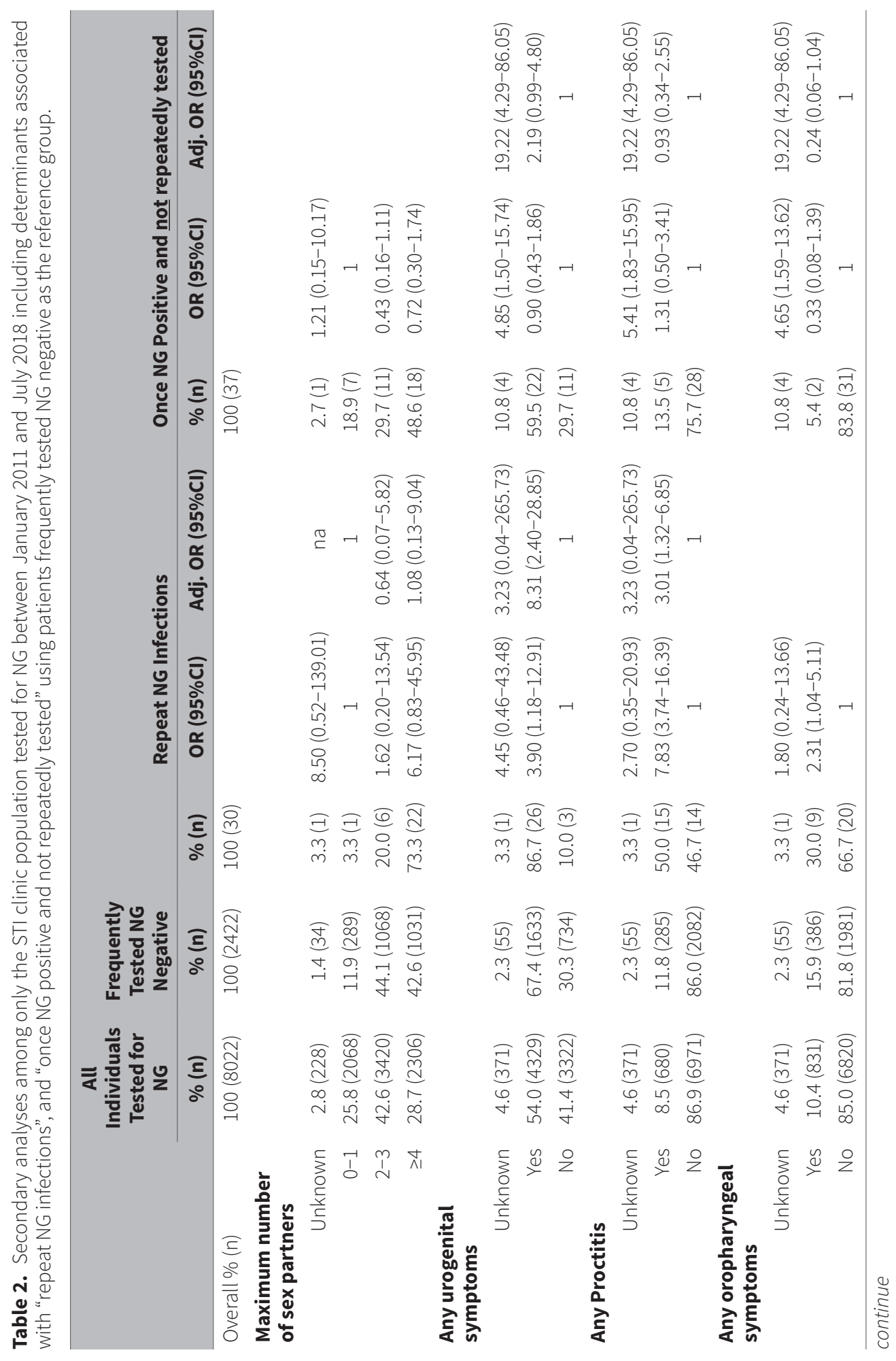




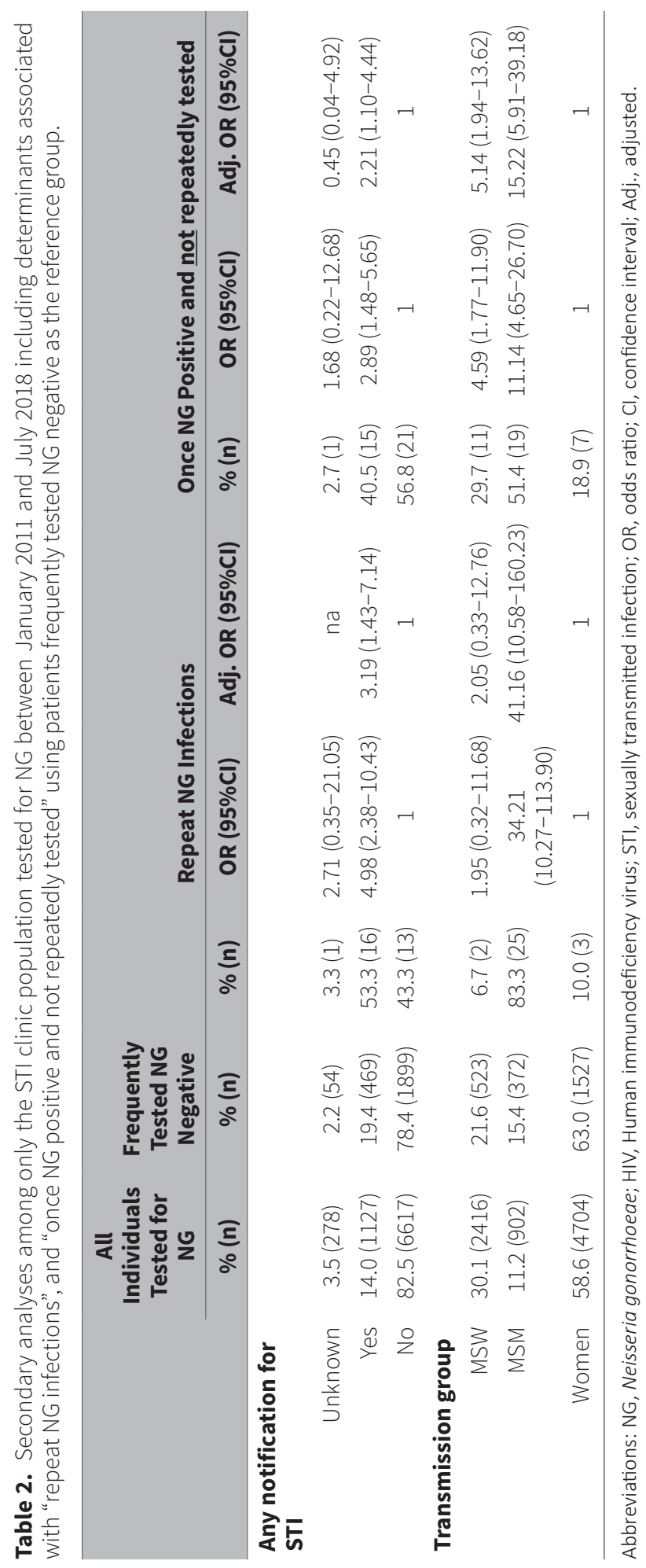




\section{Characteristics of patients once tested NG positive and not retested}

The characteristics of patients who tested once NG positive and who were not retested $(n=$ 92) were compared to the characteristics of patients who repeatedly tested negative for NG ( $n$ =4518). In multivariable analyses, these patients less often visited the STI clinic or hospital, thus more often visited the GP. They were more often men, not tested for HIV or HIV positive (Table 1).

In our secondary analyses among STI clinic visitors, patients once NG positive and not retested $(n=37$ ) were more often notified for STIs, MSW or MSM (Table 2).

\section{Geographical mapping}

The four-digit postal code of the patient was used to explore the location of patients infected with NG for potential outreach activities.

For the STI clinic population, three local areas were visualized where $\geq 3$ patients with repeat NG infections reside (Figure 2A). Two local areas nearby the STI clinic were visualized where $\geq 4$ patients, who tested positive and who were not retested, reside (Figure 2B). For the GP population, seven areas were visualized where one patient with repeat NG infections reside (Figure $2 \mathrm{C}$ ). Six local areas were visualized where $\geq 4$ patients, who were NG positive and who were not retested, reside (Figure 2D). 


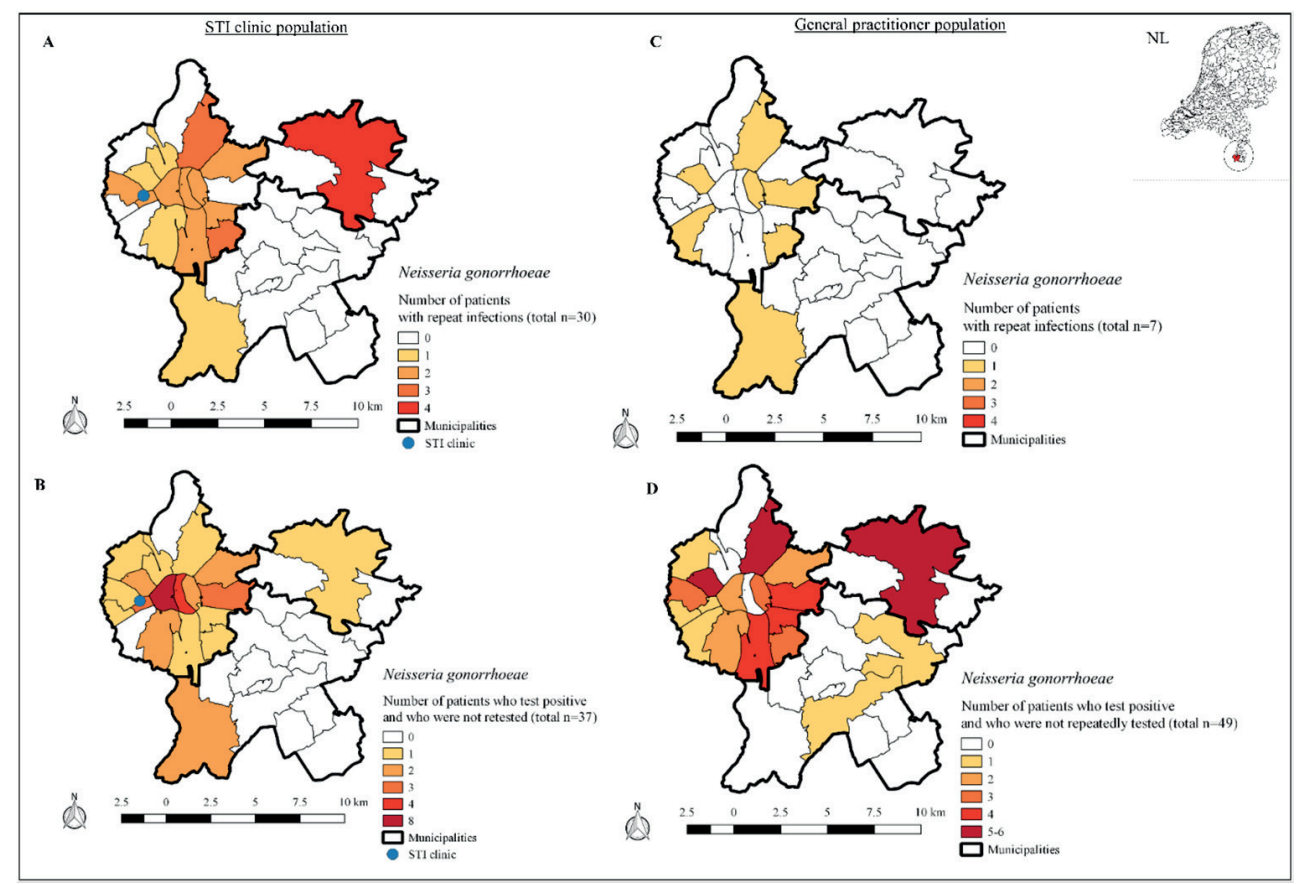

Figure 2. Geographical maps of patients infected with Neisseria gonorrhoeae. .

Geographical maps including four-digit postal code areas where patients with repeat Neisseria gonorrhoeae infections reside (A and C), and four-digit postal code areas where patients who test positive for Neisseria gonorrhoeae and who were not retested reside (B and D) stratified for the STI clinic population and general practitioner population. Location of the study area, as part of the Netherlands, is provided in the right upper corner. 


\section{Discussion}

This study provides an overview of individuals tested for NG at different STI care providers in a defined geographical area. We showed that 40 patients ( $0.2 \%$ of the patients tested for NG) were repeatedly infected with NG and accounted for one in four diagnosed NG infections. The vast majority of these patients were diagnosed at the STI clinic and were MSM. These patients are likely at highest risk for acquiring and transmitting NG. Nevertheless, focus should also be on men (including mostly MSM, but also MSW), patients co-infected with HIV, and patients notified for STIs, as they were more often once NG positive without repeat testing, potentially leading to missed repeat NG infections and ongoing transmission of NG.

Strengths of the study are the inclusion of all NG consultations of STI care providers in a defined geographical area to obtain insight in transmission of NG. Due to inclusion of all tests by the STI clinic and hospital specialists and the high coverage of GP data (81\%), underestimations of NG tests seem unlikely. ${ }^{7}$ A further strength is the timeframe of seven and a half years to partly prevent underestimations of repeat infections. ${ }^{8}$ The additional analyses among STI clinic visitors allowed the assessment of additional sexual behavior determinants to draw conclusions specified for STI clinic visitors. Furthermore, we visualized geographical areas where patients with repeat NG infections and patients who were not retested after NG diagnosis reside. Such geographical visualizations could inform local outreach activities and targeted testing.

A limitation of the study was that information on reasons for testing was unavailable. These may include financial reasons. For example, STI tests at the GP are within patients' deductibles in healthcare insurance, whereas STI tests at the STI clinic are free of charge for risk groups like young people (aged < 25 years), MSM, and commercial sex workers. Such reasons for testing could provide more insight into why patients are (not) repeatedly tested. Furthermore, the additional determinants (sexual risk behavior and symptoms) assessed for the STI clinic population were not available for the mental healthcare, GP, and hospital population. Therefore, we could not assess whether men visiting these care providers were MSM or not. However, based on additional analyses among STI clinic patients, it is likely that men diagnosed with NG by GPs are mostly MSM.

Another study observed that $28 \%$ of all diagnosed STIs were repeat ( $\geq 2)$ STIS. ${ }^{8}$ The authors concluded that a relatively small group of patients repeatedly infected with STIs likely have disproportionally high impact on circulating STIs within a population, the so called "core group". They included more STIs in their case definition including syphilis, NG, and CT. However, we believe core group transmission is STI specific. For example, $76 \%$ of the NG cases in the Netherlands are diagnosed in MSM which makes core group transmission more likely as compared to CT, which is a population disease affecting the general population such as men, women, and people aged $<25$ years. ${ }^{14}$ Furthermore, the majority of patients with repeat NG infections were living in the municipality of Maastricht, which is in general a more urbanized area compared to the other municipalities, suggesting that high risk NG groups 
mainly cluster in cities. As MSM are disproportionally affected by NG in the Netherlands and Australia, and only 40 patients accounted for one in four diagnosed NG infections, core group transmission is likely. ${ }^{14,15}$

Despite patients with repeat testing and repeat infections being at high risk for transmitting and acquiring NG, we point attention to the fact that one in three NG patients were once NG positive and not retested and, therefore, lost to care. This enables ongoing transmission as repeat NG infections are common among patients retesting within one year.,13 The Centers for Disease Control and Prevention (CDC) recommends that all men and women infected with NG should be retested three months after treatment. ${ }^{4}$ Retesting NG positive patients is an effective control strategy and can be used to enhance population-based prevention. ${ }^{4}$ Despite that retesting is advised in many international guidelines ${ }^{2-4}$, retesting rates remain typically low, ${ }^{7,13}$ indicating a need for improvement among STI care providers. Moreover, areas with relatively many patients, who were not retested after NG diagnosis, were located nearby the STI clinic. This geographical information provides a window of opportunity to inform targeted testing and outreach activities.

STI clinic patients with urogenital symptoms or proctitis had more often repeat NG infections. Studies have shown a higher NG bacterial load among symptomatic men suggesting higher transmission potential and likely clinical relevance. ${ }^{16,17}$ Therefore, men with symptoms suggestive of NG should be encouraged to present early for treatment to prevent ongoing transmission. ${ }^{16}$

The highest NG positivity rates are found among STI clinic patients notified for NG $(29.8 \%$ in women, $19.0 \%$ in heterosexual men, and 30.9\% in MSM). ${ }^{14}$ Notably, STI clinic patients notified for STIs were repeatedly infected with NG, but also lost to care indicating the essential role of partner management for targeting, testing, and treating this high-risk population.

\section{Conclusions}

Only 40 patients with repeat NG infections ( $0.2 \%$ of the individuals tested for NG) accounted for one in four NG infections. These patients were mainly STI clinic patients, MSM, and patients co-infected with CT or HIV. STI clinic patients with urogenital symptoms, proctitis, or notified for STIs had more often repeat NG infections, arguing for higher transmission potential. Therefore, it remains important to test and treat STI clinic patients, and in particular MSM for repeat NG infections. However, focus should also be on GP patients, men (including MSM and MSW), patients co-infected with HIV, and patients notified for STIS, as they were more often not retested after NG diagnosis, indicating missed repeat NG infections and ongoing transmission of NG. 


\section{References}

1. Newman, L.; Rowley, J.; Vander Hoorn, S.; Wijesooriya, N.S.; Unemo, M.; Low, N.; Stevens, G.; Gottlieb, S.; Kiarie, J.; Temmerman, M. Global Estimates of the Prevalence and Incidence of Four Curable Sexually Transmitted Infections in 2012 Based on Systematic Review and Global Reporting. PLOS ONE 2015, 10, e0143304, doi:10.1371/journal.pone.0143304.

2. Bignell, C.; Unemo, M.; European, S.T.I.G.E.B. 2012 European guideline on the diagnosis and treatment of gonorrhoea in adults. Int. J. STD AIDS 2013, 24, 85-92, doi:10.1177/0956462412472837.

3. Lazaro, N. Sexually Transmitted Infections in Primary Care 2013 (RCGP/BASHH). Available online: www.rcgp.org and www.bashh.org/guidelines (accessed on 14-10-2019).

4. Workowski, K.A. Centers for Disease Control and Prevention Sexually Transmitted Diseases Treatment Guidelines. Clin. Infect. Dis. 2015, 61 (Suppl. 8), S759-S762, doi:10.1093/cid/civ771.

5. Hosenfeld, C.B.; Workowski, K.A.; Berman, S.; Zaidi, A.; Dyson, J.; Mosure, D.; Bolan, G.; Bauer, H.M. Repeat infection with Chlamydia and gonorrhea among females: A systematic review of the literature. Sex. Transm. Dis. 2009, 36, 478-489, doi:10.1097/OLQ.0b013e3181a2a933.

6. Fung, M.; Scott, K.C.; Kent, C.K.; Klausner, J.D. Chlamydial and gonococcal reinfection among men: A systematic review of data to evaluate the need for retesting. Sex. Transm. Infect. 2007, 83, 304309, doi:10.1136/sti.2006.024059.

7. Wijers, J.; van Liere, G.; Hoebe, C.; Cals, J.W.L.; Wolffs, P.F.G.; Dukers-Muijrers, N. Test of cure, retesting and extragenital testing practices for Chlamydia trachomatis and Neisseria gonorrhoeae among general practitioners in different socioeconomic status areas: A retrospective cohort study, 2011-2016. PLoS ONE 2018, 13, e0194351, doi:10.1371/journal.pone.0194351.

8. Hsu, K.K.; Molotnikov, L.E.; Roosevelt, K.A.; Elder, H.R.; Klevens, R.M.; DeMaria, A., Jr.; Aral, S.O. Characteristics of Cases With Repeated Sexually Transmitted Infections, Massachusetts, 20142016. Clin. Infect. Dis. 2018, 67, 99-104, doi:10.1093/cid/ciy029.

9. Gesink, D.C.; Sullivan, A.B.; Miller, W.C.; Bernstein, K.T. Sexually transmitted disease core theory: Roles of person, place, and time. Am. J. Epidemiol. 2011, 174, 81-89, doi:10.1093/aje/kwr035.

10. Gotz, H.M.; van Oeffelen, L.A.; Hoebe, C.; van Benthem, B.H. Regional differences in chlamydia and gonorrhoeae positivity rate among heterosexual STI clinic visitors in the Netherlands: Contribution of client and regional characteristics as assessed by cross-sectional surveillance data. BMJ Open 2019, 9, e022793, doi:10.1136/bmjopen-2018-022793.

11. Rose, S.B.; Garrett, S.M.; Stanley, J.; Pullon, S.R.H. Retesting and repeat positivity following diagnosis of Chlamydia trachomatis and Neisseria gonorrhoea in New Zealand: A retrospective cohort study. BMC Infect. Dis. 2017, 17, 526, doi:10.1186/s12879-017-2635-y.

12. Statistics Netherlands. [Availabe online: https://www.cbs.nl/en-gb] (accessed on 22-10-2019).

13. QGIS Development Team 2019. QGIS Geographic Information System. Open Source Geospatial Foundation Project. Available online: https://qgis.org (accessed on 04-11-2019).

14. Slurink, I.; van Aar, F.; Op de Coul, E.; Heijne, J.; van Wees, D.; Hoenderboom, B.; Visser, M.; den Daas, C.; Woestenberg, P.; Gotz, H.; et al. Sexually transmitted infections in the Netherlands in 2018; 
Rijksinstituut voor Volksgezondheid en Milieu: Utrecht, The Netherlands, 2019; doi: 10.21945/rivm2019-0007.

15. Cornelisse, V.J.; Zhang, L.; Law, M.; Chen, M.Y.; Bradshaw, C.S.; Bellhouse, C.; Fairley, C.K.; Chow, E.P.F. Concordance of gonorrhoea of the rectum, pharynx and urethra in same-sex male partnerships attending a sexual health service in Melbourne, Australia. BMC Infect. Dis. 2018, 18, 95, doi:10.1186/ s12879-018-3003-2.

16. Priest, D.; Ong, J.J.; Chow, E.P.F.; Tabrizi, S.; Phillips, S.; Bissessor, M.; Fairley, C.K.; Bradshaw, C.S.; Read, T.R.H.; Garland, S.; et al. Neisseria gonorrhoeae DNA bacterial load in men with symptomatic and asymptomatic gonococcal urethritis. Sex. Transm. Infect. 2017, 93, 478-481, doi:10.1136/ sextrans-2016-052950.

17. Bissessor, M.; Tabrizi, S.N.; Fairley, C.K.; Danielewski, J.; Whitton, B.; Bird, S.; Garland, S.; Chen, M.Y. Differing Neisseria gonorrhoeae bacterial loads in the pharynx and rectum in men who have sex with men: Implications for gonococcal detection, transmission, and control. J. Clin. Microbiol. 2011, 49, 4304-4306, doi:10.1128/JCM.05341-11. 


Section bacterial load 


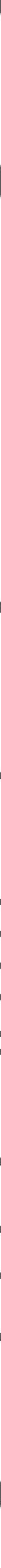




\section{Abstract}

\section{Background}

The bacterial load of Chlamydia trachomatis (CT) is assumed to play a role in transmission and sequelae. We assessed urogenital CT cycle quantification (Cq) values, as an indicator for CT load, of men and women diagnosed by general practitioners (GPS), hospital physicians and the STI clinic.

\section{Methods}

Urogenital CT-positive samples ( $n=2,055$ vaginal swabs, $n=77$ cervix swabs, $n=1,519$ urine samples and $n=19$ urethral swabs) diagnosed by GPs, hospital physicians and the STI clinic from the Maastricht Medical Microbiology Laboratory were included (2012-2016). The outcome measure 'urogenital Cq values' was used as an inversely proportional measure for CT load. Among all patients, multivariate linear regression analyses were used to assess primary determinants for mean urogenital $\mathrm{Cq}$ values, stratified by sex. Additional clinical determinants were assessed among STI clinic patients.

\section{Results}

In men, mean urogenital Cq values were similar between GPS, hospital physicians and the STI clinic (32.7 and 33.5 vs. 32.7; p>0.05). Women visiting the GP had lower urogenital Cq values than women visiting the STI clinic (30.2 vs. 30.9; $p=<0.001$ ). Women visiting the hospital had higher urogenital Cq values than women visiting the STI clinic (32.4 vs. 30.9; $p=<0.001$ ). Among STI clinic women, urogenital Cq values were lower in women with concurrent anorectal CT and in rectally untested women compared to anorectal CT-negative women (30.7 and 30.6 vs. 33.9; $p=<0.001)$.

\section{Conclusion}

Men visiting different STI care providers had similar urogenital Cq values, which could be an indicator for similar CT loads. The lower Cq values of women visiting the GP compared to women visiting the STI clinic could be an indicator for higher CT loads and likely higher transmission potential. Notably, urogenital Cq values of STI clinic women were much lower (>3 Cq) when STI clinic women also had anorectal CT. This finding could indicate higher urogenital CT loads and likely higher chances of transmission and sequelae. 


\section{Introduction}

Chlamydia trachomatis (CT) is the most prevalent bacterial sexual transmitted infection (STI) worldwide. ${ }^{1} \mathrm{CT}$ infection can increase the risk of reproductive sequelae in women, such as pelvic inflammatory disease, infertility, and ectopic pregnancy. ${ }^{2}$

In several countries, such as Australia, the United Kingdom and the Netherlands, general practitioners (GPS) have a significant role in STI healthcare, as a large proportion of CT infections are diagnosed by GPs. ${ }^{3-6}$ Other STI care providers include STI clinics, genitourinary medicine (GUM) clinics and hospital physicians. ${ }^{578} \mathrm{STI}$ care providers test different populations of patients. Studies have shown differences in age, sex, race and socioeconomic (SES) characteristics of patients visiting different STI care providers. ${ }^{7-9}$ CT-positive patients visiting different STI care providers also differ in characteristics such as age, sex, race and SES. ${ }^{78}$ Potentially CT-positive patients of different STI care providers also differ in CT bacterial load (hereafter CT load).

The CT load, often expressed as the number of CT bacteria present per milliliter, has been studied over several years. ${ }^{10}$ An earlier report by our study group showed comparable urogenital CT loads in men and women participating in a Dutch population-based CT screening and STI clinic visitors, arguing similar chances of transmission and sequelae. ${ }^{11}$ Previous studies assessing CT load included separate patient populations of STI care providers, such as the STI clinic and GPs. ${ }^{1012-14}$ Nevertheless, GP, hospital and STI clinic patient populations have never before been compared regarding CT load. Comparing the CT loads of patients visiting different STI care providers could expand our understanding of CT-infected patient populations served by our STI care services.

Currently, it is not known what determines a high bacterial CT load in a patient and what its consequences are. Symptoms might be associated with higher CT loads but this remains a matter of debate. ${ }^{10}$ It could be relevant to assess determinants for high CT loads. For example, in viral STIs, such as herpes simplex virus and human immunodeficiency virus (HIV), it has been shown that higher viral loads increase transmission potential. ${ }^{10}$ However, it is unknown whether this also applies to CT load. ${ }^{10}$

Concurrent urogenital and anorectal infections are common among women visiting the STI clinic, i.e. more than $70 \%$ of women with urogenital infections also have an anorectal infection. ${ }^{15}{ }^{16}$ Current guidelines advocate anorectal testing in women based on indication, i.e. after self-report of anal sex and/or symptoms. ${ }^{15}$ However, as GPs rarely test women anorectally it remains unknown whether anorectal infections are common among women visiting the GP. ${ }^{17}$

Our main objective was to compare the urogenital CT Cq values, as an indicator for CT load, between CT-positive patients tested by GPS, hospital physicians and the STI clinic to obtain more insight in the CT loads of different populations. Our second objective was to assess which clinical determinants were associated with urogenital Cq values. 


\section{Methods and materials}

\section{Ethics statement}

The medical ethics committee of the Maastricht University Medical Center (Maastricht, the Netherlands) approved this study (METC 2017-0251) and waived the need for consent to be collected from participants. Since retrospective data originated from regular care and were analyzed anonymously, no further informed consent for data analysis was obtained.

\section{Study population}

In this cross-sectional study, data from 3,899 test consultations of urogenital CT-positive patients $\geq 16$ years (from $n=38,599$ consultations; $10.1 \%$ CT positive) were obtained from the Medical Microbiology Laboratory of Maastricht University Medical Center (MUMC+) from January 2012 through May 2016. Data included consultations performed by GPs, hospital physicians and the STI clinic. The majority of hospital consultations were performed by gynecologists (42,5\%; $n=48$ ) followed by internists (22.1\%; $n=25)$. The proportions of urogenital CT tests performed by the GP, hospital physicians and STI clinic do not reflect the real distribution of STI care provider testing in our region, as we included different geographic serving areas for the STI care providers.

The laboratory provides the same instruction methods for collecting CT samples for GPS, hospital physicians and STI clinic using the same sampling materials. The CT samples were daily transported to the laboratory. All samples were collected in a standardized way and were analyzed in the same laboratory.

All samples were tested for CT with a nucleic acid amplification test (NAAT) (COBAS 4800, Roche Diagnostics, Basel, Switzerland), as per the manufacturer's protocol. ${ }^{11}$ The NAAT-derived cycle quantification $(\mathrm{Cq})$ value is commonly used as a proxy for bacterial load in other infectious diseases; a low Cq value indicates a high load, and vice versa. ${ }^{18-20}$

The study population included data from all samples for which the Cq value could be retrieved $(98.0 \% ; 3,821 / 3,899)$ (Figure 1). For men, data from urine and urethral swabs were available. Mean Cq values from urine $(n=1,519, M=32.69, S D=3.20)$ and urethral swabs ( $n=19$, $M=32.88, S D=4.80)$ were comparable in men $(p=0.86)$. These data were merged together as 'urogenital Cq values'.

For women, data from urine, vaginal swabs and cervix swabs were available. Mean Cq values from vaginal swabs ( $n=1,973, M=30.86, S D=3.73$ ) and cervix swabs ( $n=77, M=30.58$, $\mathrm{SD}=4.24)$ were comparable in women ( $p=0.51)$. The data were merged together as 'urogenital Cq values'. Mean Cq values from vaginal swabs ( $n=1,973, M=30.86, S D=3.73$ ) and urine ( $n=233$, $M=33.28, S D=3.60)$ were significantly different $(p=<0.001)$. Data from urine samples of women 
were excluded ( $n=233$ ), as international guidelines advocate vaginal swabs, as the diagnostic value (sensitivity and specificity) for detecting CT in urine samples is lower compared to vaginal swabs. ${ }^{21-24}$ The majority of urine samples from women were taken by GPs (94\%; Figure 1).

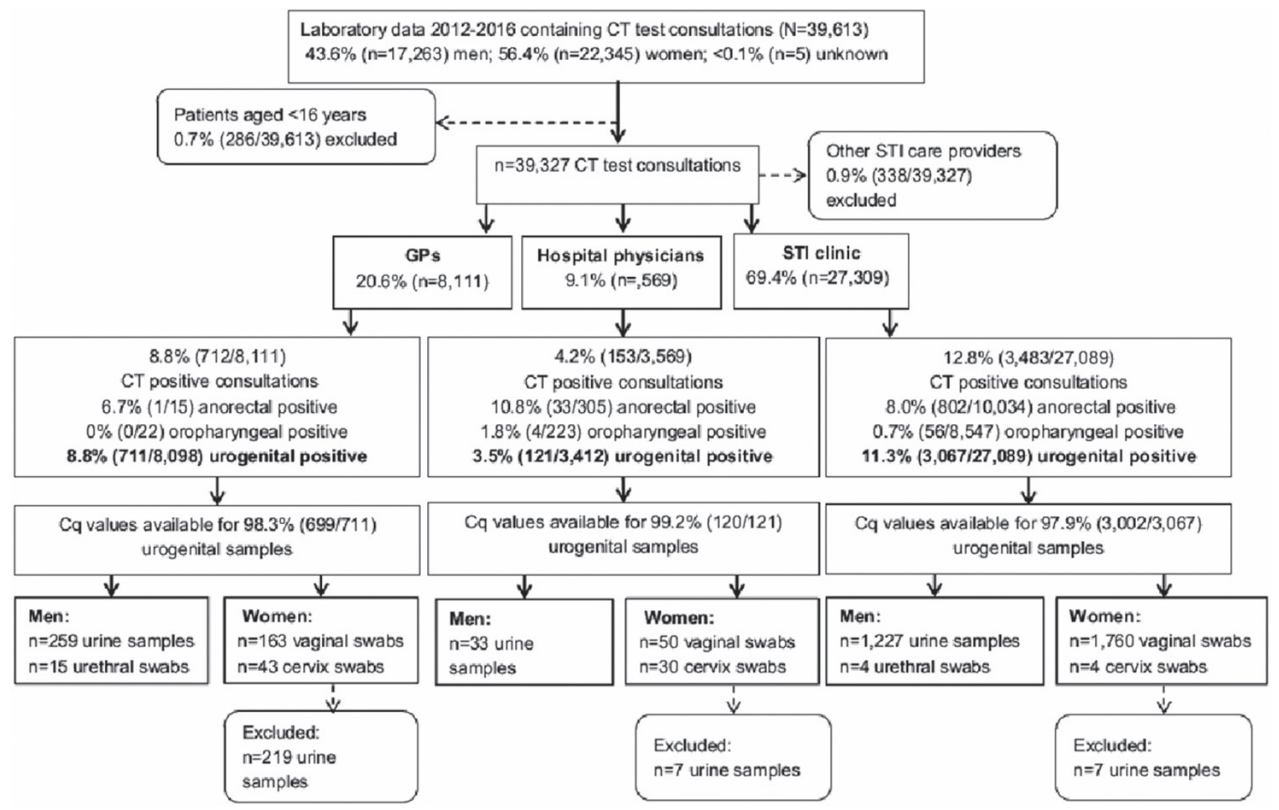

Figure 1. Flowchart, including Chlamydia trachomatis samples taken by general practitioners, hospital physicians and the STI clinic between January 2012 and May 2016.

\section{Cycle quantification validation for CT load}

We tested the use of the Cq value as a proxy for CT load by comparing derived Cq values with quantified CT load values from our previous studies. ${ }^{112526} \mathrm{CT}$ load values were available from a subset of the STI clinic population, i.e. $n=103$ vaginal swabs from women (S1 Table). In short, we quantified CT load by an in-house TaqMan real-time qPCR to quantify CT OmpA-gene copies/ml. ${ }^{11} \mathrm{~A}$ full description of the CT load quantification has been described elsewhere. ${ }^{11}$

S2 Figure shows the high correlation between vaginal Cq values and vaginal CT load (CT/ $\mathrm{ml} \log 10)$ (Pearson's $\mathrm{r}$ - $0.80, \mathrm{n}=103, \mathrm{p}=<0.001$ ). Therefore, the $\mathrm{Cq}$ value is a valid inversely proportional proxy for CT load. 


\section{Statistical analyses}

The main objective was to compare the outcome measure, i.e. 'urogenital Cq values', between the populations visiting different STI care providers. Therefore, the main determinant was STI care provider (GP, hospital physician, or STI clinic).

Analyses were stratified for men and women since CT load varies by sample type and sex. ${ }^{10}$ Baseline characteristics were compared between the GP, hospital physician and the STI clinic CT-positive populations using chi-square tests. Univariable and multivariable linear regression analyses were performed to test the association between the main determinant and the outcome, controlling for putative confounders. The putative confounders were available for the whole study population and included age in years $(<25, \geq 25$ ), SES (low, medium, high, unknown), Neisseria gonorrhoeae (NG) urogenital positive (yes, no, not tested) and HIV positivity (yes, no, not tested). Dutch SES scores based on income, education level and employment were extracted from the Netherlands Institute for Social Research (http://www.scp.nl) per four-digit postal code area of the patient.

Our second objective was to assess potential associations between the outcome measure and clinical determinants (available for the STI clinic population), including concurrent anorectal CT infection (no anorectal test, yes, no), urogenital symptoms (unknown, yes, no), oropharyngeal symptoms (unknown, yes, no), proctitis (unknown, yes, no) and, for men, sexual preference (unknown, MSM, heterosexual men).

For all linear regression analyses, determinants with $p<0.05$ in the univariable model were included in the multivariable model. To test our main objective, the main determinant 'STI care provider' was entered in the multivariable model. Means, betas and $95 \%$ confidence intervals (CI) were calculated. Finally, the proportions of low and high Cq values were assessed between the STI care providers based on quartiles and are depicted in Figure 2. Analyses were performed using SPSS V21 (IBM SPSS Statistics for Windows, IBM Corporation, Armonk, New York, USA). A p value of $<0.05$ was considered statistically significant.

\section{Results}

\section{Study population}

Of the 3,588 urogenital samples in the dataset, $42.9 \%$ ( $n=1,538)$ were samples from men, and $57.1 \%(n=2,050)$ were samples from women. Baseline characteristics (age, SES, NG and HIV) differed between the CT-positive populations visiting GPs, hospital physicians and the STI clinic in both men and women (Table 1). 


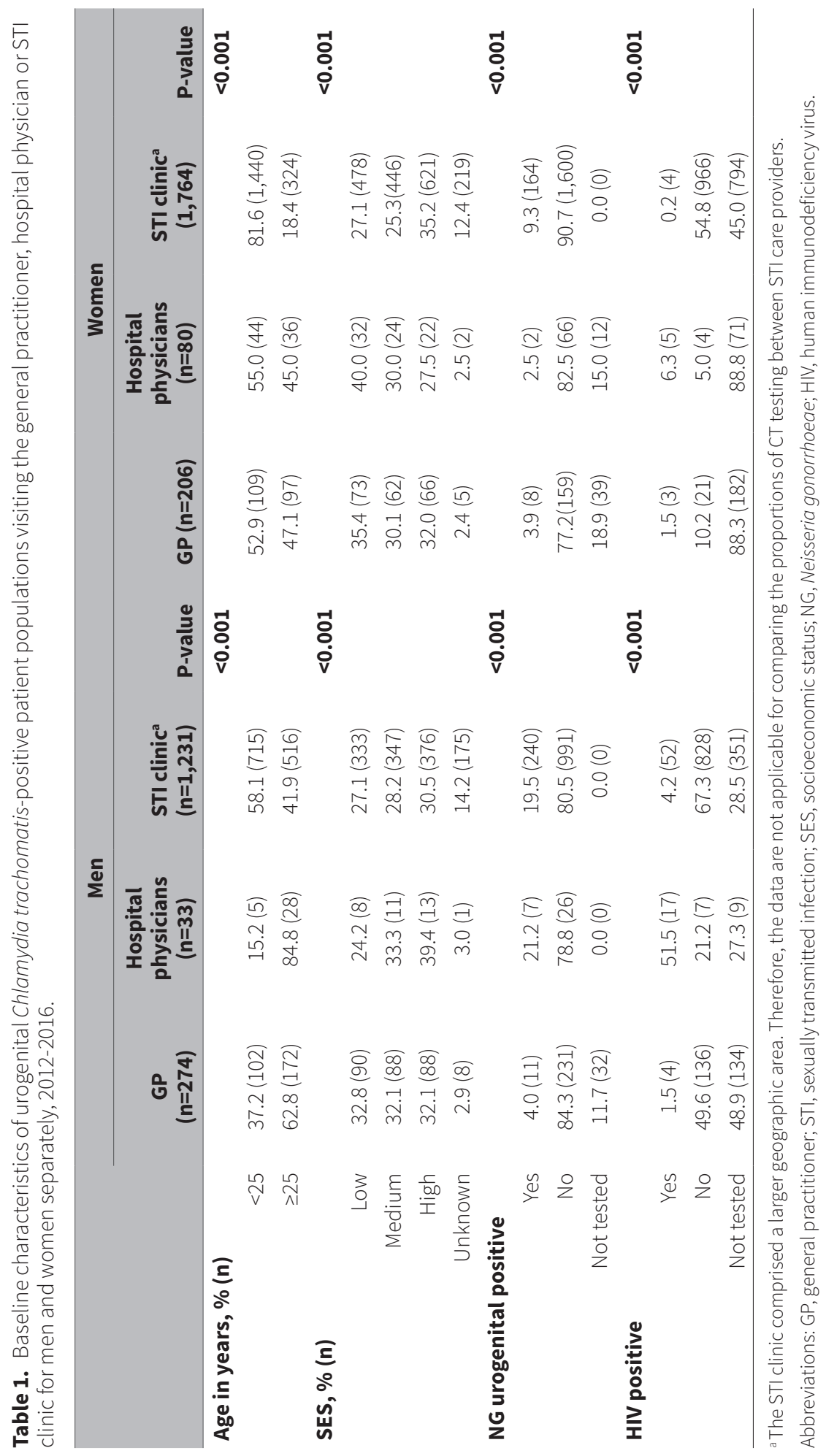




\section{Urogenital $\mathrm{Cq}$ values of $\mathrm{CT}$ patients compared between STI care providers}

In multivariable analyses, mean urogenital $\mathrm{Cq}$ values were similar in men diagnosed by GPS ( $M=32.7, S D$ 3.2), hospital physicians ( $M=33.5, S D=3.5)$ and the STI clinic ( $M=32.7, S D=3.2)$ $(p=0.36)$ (Figure 2 and Table 2). Based on quartiles, the proportions of low urogenital Cq values were similar among men visiting GPs (26.3\%), hospital physicians (24.2\%) and the STI clinic (24.2\%) (Figure 2).

In multivariable analyses, urogenital Cq values were lower for women visiting the GP compared to women visiting the STI clinic ( $M=30.2, S D=4.2$ vs. $M=30.9, S D=3.7, p<0.001)$. Urogenital $\mathrm{Cq}$ values were higher for women visiting hospital physicians ( $M=32.4, S D=4.3, p<0.001$ ) compared to women visiting the STI clinic. Based on quartiles, the proportion of low urogenital Cq values of CT positive women was higher for GPs (35.9\%) compared to hospital physicians (18.8\%) and the STI clinic (23.2\%) (Figure 2).

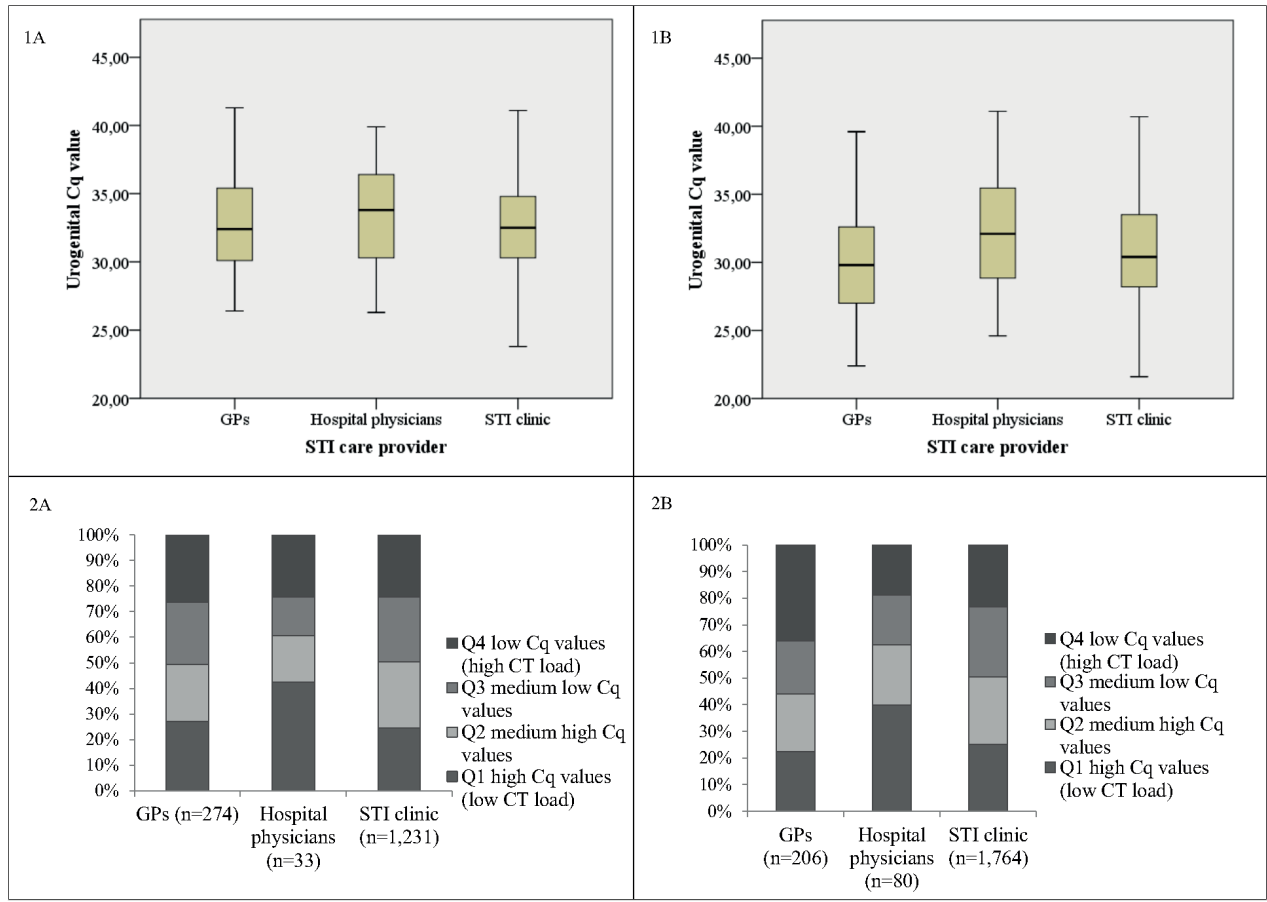

Figure 2. Boxplots and bar diagrams showing the distribution of high- and low Cq values for the GP, hospital and STI clinic population..

(1) Boxplots showing the distribution of urogenital Cq values between the GP, hospital physicians and STI clinic population for men (A) and women (B). (2) Bar diagrams showing the frequencies of low (high CT load), medium low, medium high and high (low CT load) urogenital Cq values based on quartiles per STI provider for men (A) and women (B). 


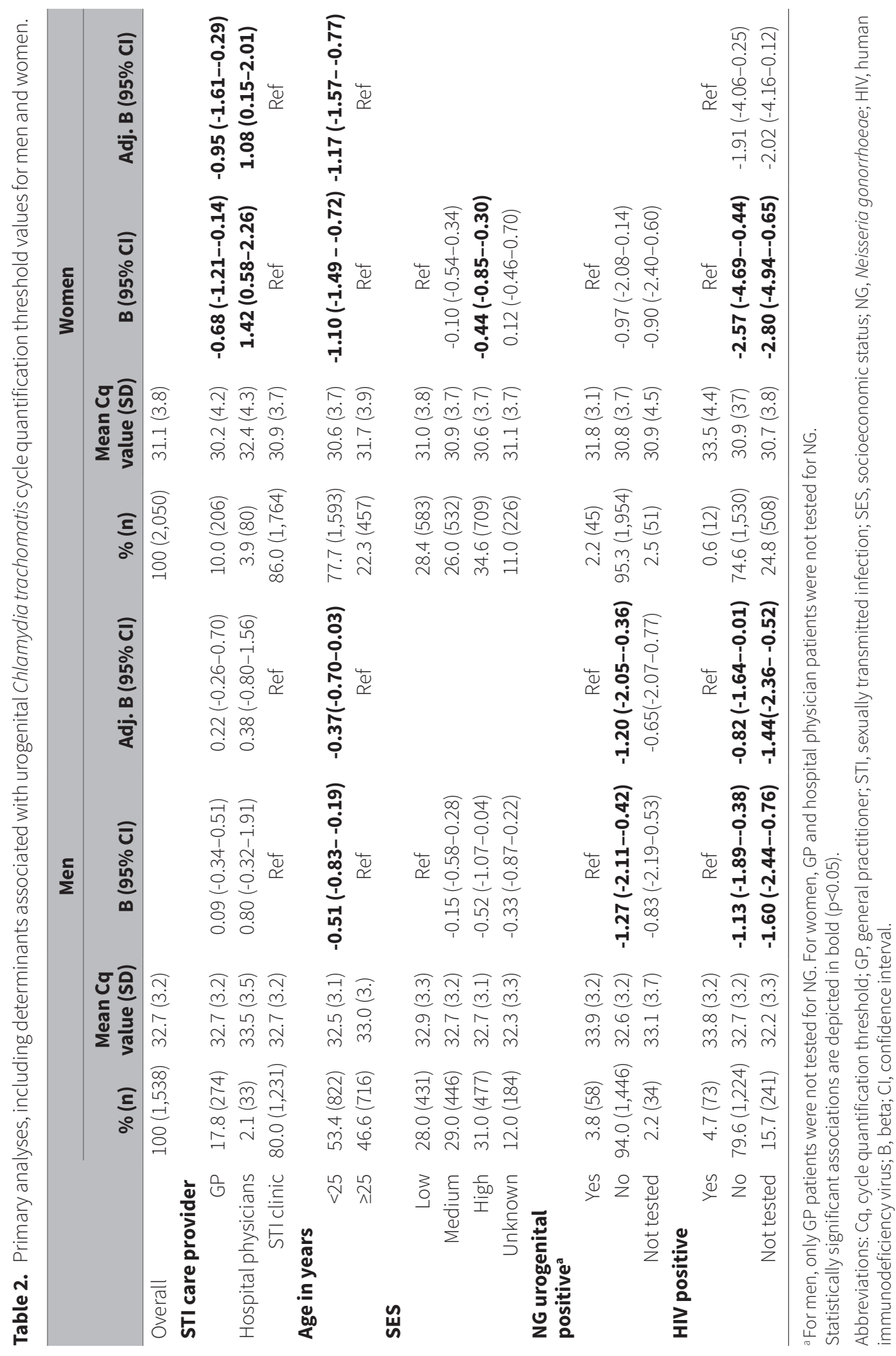




\section{Other determinants associated with urogenital Cq values}

In multivariable analyses, age $<25$ years (compared to age $\geq 25$ years), no concurrent NG (compared to concurrent NG), HIV negative and not being tested for HIV (compared to HIV positive) were associated with lower Cq values in men (Table 2).

Furthermore, age $<25$ years (compared to age $\geq 25$ years) was associated with lower $\mathrm{Cq}$ values in women (Table 2).

\section{Clinical determinants in STI clinic patients}

In multivariable analyses, having no concurrent NG (compared to NG positive), not being tested for HIV (compared to HIV positive) and having urogenital symptoms (compared to having no urogenital symptoms) were associated with lower urogenital Cq values in men (Table 3).

Furthermore, age $<25$ years (compared to age $\geq 25$ years), having no anorectal $\mathrm{CT}$ test and having a concurrent anorectal CT infection (compared to no anorectal CT infection) were associated with lower $\mathrm{Cq}$ values in women (Table 3). 


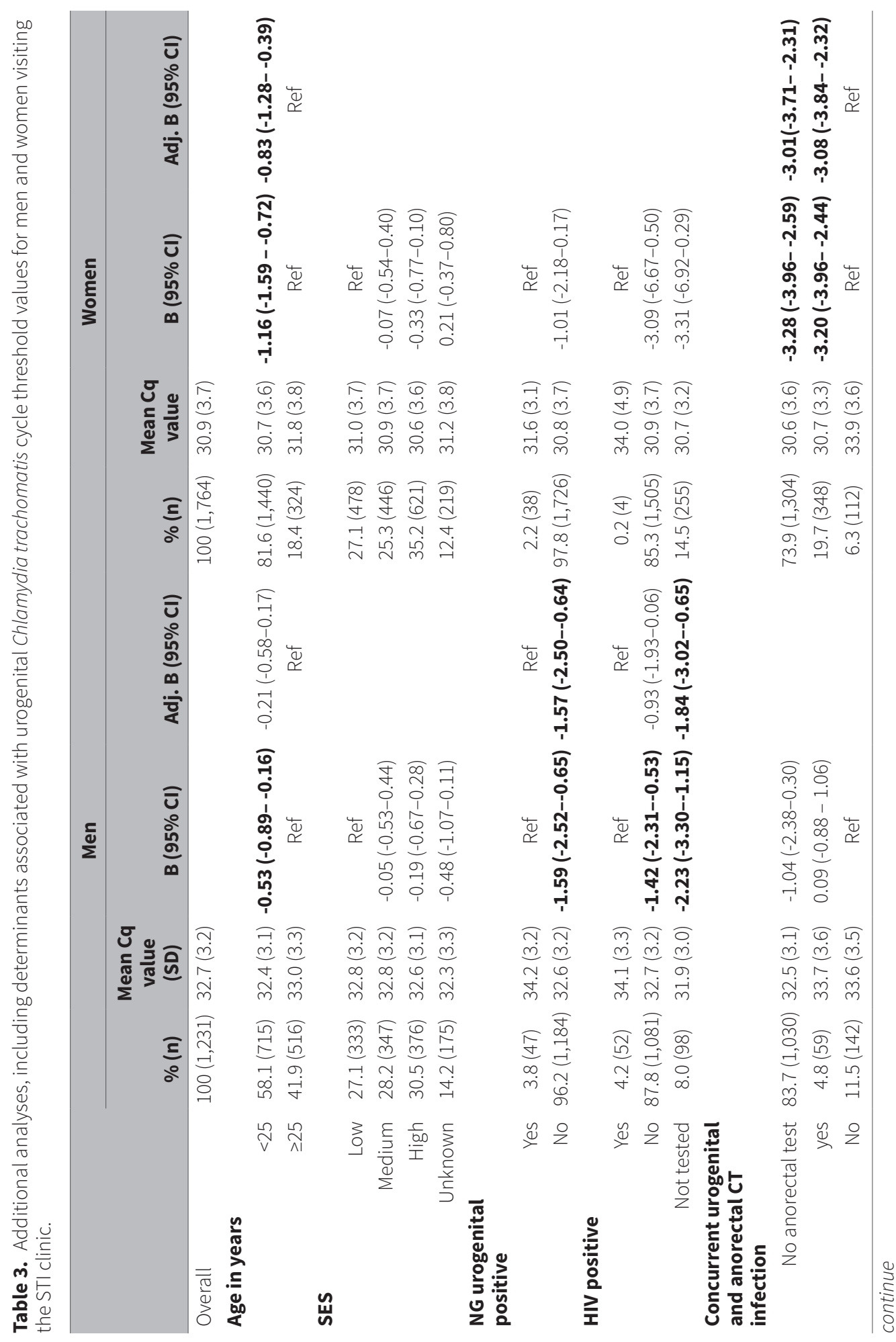




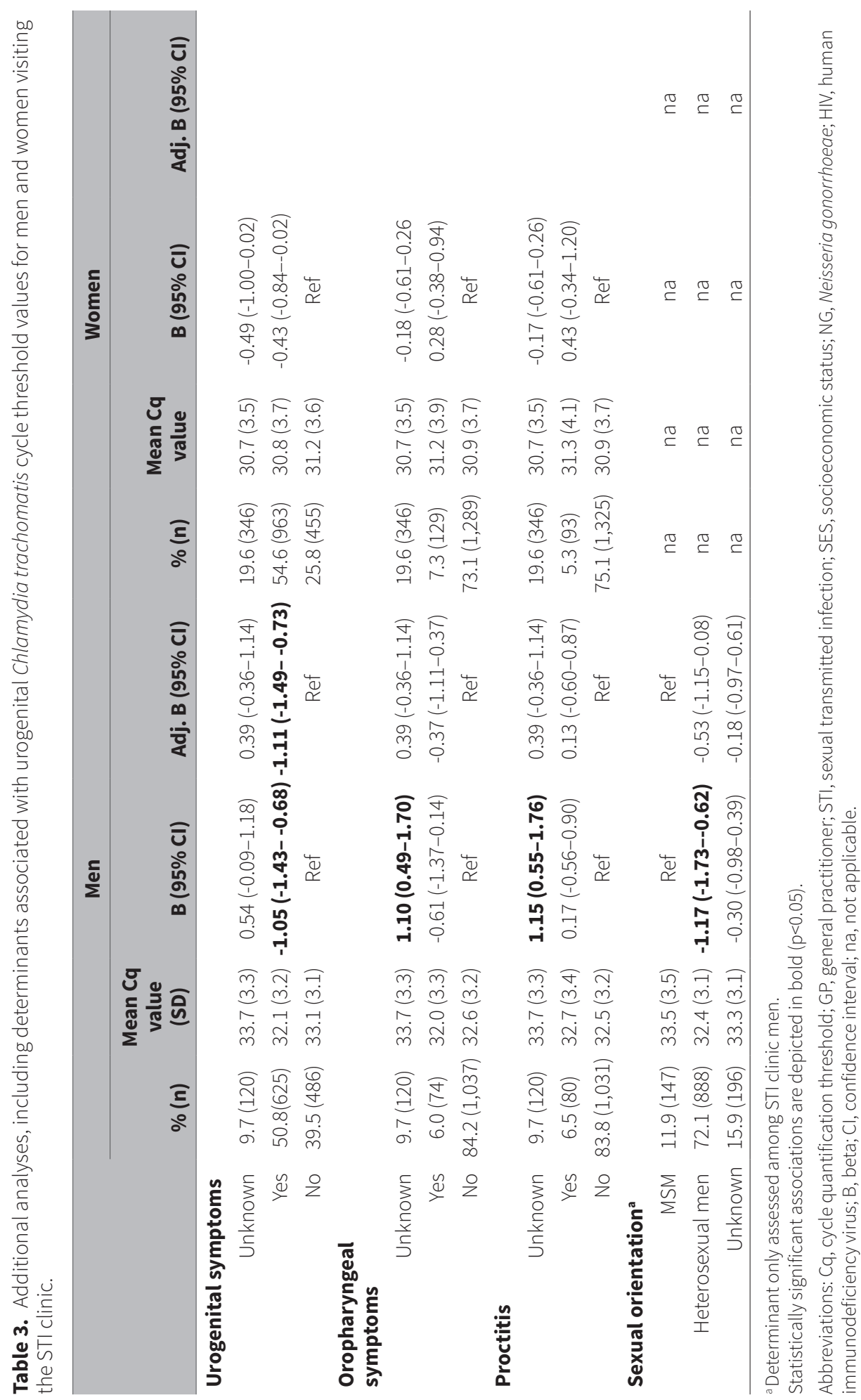




\section{Discussion}

To date, our study is the first to compare urogenital Cq values, as a potential indicator for CT load, of men and women diagnosed by GPs, hospital physicians and the STI clinic. Among all STI care providers, men visiting GPS, hospital physicians and the STI clinic had similar urogenital Cq values and likely similar urogenital CT loads. Women diagnosed by GPs had lower Cq values than women visiting the STI clinic, which could be indicative for higher urogenital CT loads. Whereas women visiting the hospital had higher urogenital Cq values than women visiting the STI clinic; likely indicative for lower CT loads. Of all determinants studied, only a few determinants were statistically associated with urogenital Cq values. However, one determinant, assessed among STI clinic visitors, was notable as the adjusted mean difference was much higher (> $3 \mathrm{Cq}$ ) compared to all other determinants.

A strength of the current study is the large number of included samples from different STI care providers. Furthermore, the additional analyses on clinical determinants in the STI clinic population allowed us to increase our understanding of what type of patients have lower urogenital $\mathrm{Cq}$ values and, therefore, likely higher urogenital CT loads. Furthermore, vaginal $\mathrm{Cq}$ values and vaginal CT loads were highly correlated. Therefore, vaginal $\mathrm{Cq}$ values were a valid indicator for vaginal CT loads.

A general limitation of CT load-based studies is that the variability of low and high CT loads, and therefore high and low Cq values, in populations and individuals seems dependent of different factors, including time of diagnosis since infection; this hampers interpretation. For example, our study group assessed the natural course of the CT load during infection between screening and treatment and observed a decrease in CT load in 17-41\% of the STI clinic patients dependent on sample type. ${ }^{26} \mathrm{~A}$ limitation of the current study was that we were unable to validate $\mathrm{Cq}$ values for (1) other populations than the STI clinic population and (2) for urine samples of men. Therefore, it remains unclear whether Cq values of the GPand hospital population are a valid proxy for CT load in GP- and hospital populations and whether $\mathrm{Cq}$ values are a valid indicator for CT loads in urine samples. Our outcome measure 'urogenital Cq values' provides an indication of CT load. However, estimating the number of gene copy numbers per milliliter (often expressed as CT/milliliter) would have led to more accurate estimates of CT load. The Cq values in the current study are not reproducible for all NAAT used for CT detection. For example, if our study was repeated with a Siemens Versant NAAT, different Cq values could be produced. However, the relative differences between the Cq values of, for example, the GP, hospital and STI clinic population will be the same. Furthermore, we were unable to assess whether symptoms (urogenital symptoms, oropharyngeal symptoms and proctitis) were a result of a CT infection. Therefore, the symptoms could be caused by other STI's such as herpes simplex virus, Mycoplasma genitalium or Trichomonas vaginalis. However, as those STI's occur to a much lesser extent among STI clinic visitors in the Netherlands the influence seems to be low. ${ }^{27} 28$ Furthermore, as we used urogenital Cq 
values as a proxy for CT load caution is needed when comparing our results to studies that used the actual CT load as an outcome measure.

The purpose of the current study was to provide insight in the CT load of populations visiting different STI care providers. The clinical relevance of our main finding, suggesting different CT loads between women visiting the GP, hospital and STI clinic, remains debatable as the exact role of CT load remains unknown. ${ }^{10}$ Therefore, results of the current study will not lead to clinical consequences. Earlier, our study group deemed a difference of 1 log load $(3.3 \mathrm{Cq})$ as clinically relevant to overcome potential technical variations when measuring the CT load within the same patient over time. ${ }^{26}$ However, in the current study we averaged $\mathrm{Cq}$ values over an entire population, i.e. GP, hospital and STI clinic population. Therefore, even a smaller difference than $3.3 \mathrm{Cq}$ could be clinically or microbiologically relevant. Still, the exact cut-off value for a relevant difference in CT load between populations remains unknown.

One determinant showed an adjusted difference of $3.08 \mathrm{Cq}$, related to anorectal CT, which was much higher than all other determinants. Dubbink and colleagues also observed a higher urogenital CT load among South-African women concurrently infected with anorectal CT.29 It is likely that the majority of STI clinic women who were not anorectally tested also had an anorectal infection since more than 70\% of the STI clinic women with a urogenital infection also have a concurrent anorectal infection. ${ }^{15} 16$ Indeed, urogenital Cq values were comparable for STI clinic women who had diagnosed concurrent urogenital and anorectal CT infections and STI clinic women who were not anorectally tested. Those STI clinic women who were only urogenitally CT positive and not anorectally tested were likely treated with azithromycin, as azithromycin is the first choice treatment for urogenital CT in the Netherlands. ${ }^{30}$ Therefore, possible undiagnosed concurrent anorectal CT infections could be not adequately treated, as the efficacy of azithromycin, compared to doxycycline, may be lower for anorectal CT infections. ${ }^{2331}$ Likely women with concurrent urogenital and anorectal CT infections could have higher transmission potential, yet this remains unclear. Recently, our study groups showed a borderline significant association ( $P=0.054$ ) between lower vaginal $\mathrm{Cq}$ values, i.e. likely higher $\mathrm{CT}$ loads, and not reaching microbiological cure for vaginal CT in women treated with azithromycin. ${ }^{32}$ Therefore, some women with concurrent vaginal and (untested) anorectal infections in our study may not be microbiologically cured when treated with azithromycin as they tend to have lower Cq values and therefore potentially higher CT loads. ${ }^{32}$ Notably, anorectal testing was rarely performed by GPs and hospital physicians (Figure 1), as has also been shown before. ${ }^{81733}$ However, according to international guidelines, anorectal testing should at least be performed in men and women reporting anorectal intercourse or symptoms. ${ }^{2324}$ Still, it remains unknown to which extent women visiting the GP report anal intercourse. However, it is unlikely that this would be $0.2 \%$, as a population based study showed that $10.5 \%$ of women report to have anorectal intercourse in the past year. ${ }^{34}$

Two explanations could possibly explain the lower Cq values of women visiting the GP compared to women visiting the STI clinic. First, lower urogenital Cq values of women visiting the GP could be related with symptoms, as symptoms could be associated with higher CT 
loads. ${ }^{10}$ A study by van Bergen and colleagues showed that only $20 \%$ of the patients with STI related symptoms visit the STI clinic, whereas the majority of symptomatic patients visit the GP $(63 \%) .{ }^{35}$ Second, women visiting the STI clinic could have higher Cq values, and therefore likely lower CT loads, due to frequent CT infections. It has been shown that the CT load is lower when having repeat CT infections. ${ }^{36}$ Moreover, retesting rates are higher for the STI clinic population compared to the GP population what could strengthen this explanation. ${ }^{37}$ The higher $\mathrm{Cq}$ values of women visiting the hospital may be due to different sampling moments during the infection. ${ }^{1138}$ It is likely that women visit the hospital at a later stage of the infection than women visiting the STI clinic, which could have impact on the Cq values, and therefore likely CT loads, as CT loads tend to decrease over time. ${ }^{26}$

Younger women (<25 years) had significantly lower urogenital Cq values, i.e. potentially higher CT loads, than older ( $\geq 25$ years) women. Others report that partial immunity acquired to past CT infection could possibly lead to lower CT load with increasing age.39 The lower urogenital Cq values of men without concurrent NG and men who were not tested for HIV remains unexplained. Men with urogenital symptoms had lower Cq values, i.e. likely higher urogenital CT loads, than men without urogenital symptoms. Symptoms associated with higher urogenital CT load in men have been observed in several studies. ${ }^{11} 4041$ An explanation for higher urogenital CT loads could be that higher CT loads induce a greater inflammatory response. $^{11}$

We excluded all urine samples of women ( $n=233$ ). Almost all urine samples were taken by the GP (94\%). GPs should consider collecting self-sampled vaginal swabs of women, as the sensitivity and specificity of vaginal swabs for detecting CT are much higher compared to urine samples. ${ }^{22-24}$

\section{Conclusions}

The patient characteristics of male CT patients diagnosed by GPS, hospital physicians and STI clinic differed by type of STI care provider. However, the urogenital Cq values of men were similar, arguing similar CT loads and chances of transmission and sequelae. Women visiting the GP had lower urogenital Cq values than women visiting the STI clinic, which could be an indicator for higher urogenital CT loads. Whereas women visiting the hospital had higher urogenital Cq values than women visiting the STI clinic, which could be an indicator for lower urogenital CT loads. The impact, in terms of transmission and sequelae, of lower Cq values, and likely higher CT loads, needs to be explored further. Notably, much lower urogenital Cq values, and likely higher urogenital CT loads, were observed when STI clinic women also had anorectal CT and in STI clinic women who were not anorectally tested but who are prone to have an undiagnosed anorectal infection. Likely those STI clinic women with anorectal infections have higher chances of transmission and sequelae. 


\section{References}

1. Newman L, Rowley J, Vander Hoorn S, et al. Global Estimates of the Prevalence and Incidence of Four Curable Sexually Transmitted Infections in 2012 Based on Systematic Review and Global Reporting. PLoS One 2015;10(12):e0143304. doi: 10.1371/journal.pone.0143304

2. Haggerty CL, Gottlieb SL, Taylor BD, et al. Risk of sequelae after Chlamydia trachomatis genital infection in women. J Infect Dis 2010;201 Suppl 2:S134-55. doi: 10.1086/652395

3. Bowring AL, Gouillou M, Guy R, et al. Missed opportunities--low levels of chlamydia retesting at Australian general practices, 2008-2009. Sex Transm Infect 2012;88(5):330-4. doi: 10.1136/ sextrans-2011-050422

4. Santella AJ, Pollack A, Harrison C, et al. Management rates of sexually transmissible infections by Australian general practitioners, 2000-2012. Sex Health 2014;11(1):52-7. doi: 10.1071/SH13179

5. Wetten S, Mohammed H, Yung M, et al. Diagnosis and treatment of chlamydia and gonorrhoea in general practice in England 2000-2011: a population-based study using data from the UK Clinical Practice Research Datalink. BMJ Open 2015;5(5):e007776. doi: 10.1136/bmjopen-2015-007776

6. Visser M, van Aar F, Op de Coul E, et al. Sexually transmitted infections in the Netherlands in 2017. Seksueel overdraagbare aandoeningen in Nederland in 2017: Rijksinstituut voor Volksgezondheid en Milieu RIVM.

7. Batteiger TA, Dixon BE, Wang J, et al. Where Do People Go for Gonorrhea and Chlamydia Tests: A Cross-sectional View of the Central Indiana population, 2003-2014. Sex Transm Dis 2018 doi: 10.1097/OLQ.0000000000000928

8. den Heijer CD, van Liere GA, Hoebe CJ, et al. Who tests whom? A comprehensive overview of Chlamydia trachomatis test practices in a Dutch region among different STI care providers for urogenital, anorectal and oropharyngeal sites in young people: a cross-sectional study. Sex Transm Infect 2016;92(3):211-7. doi: 10.1136/sextrans-2015-052065

9. Clifton S, Mercer CH, Woodhall SC, et al. Patterns of chlamydia testing in different settings and implications for wider STI diagnosis and care: a probability sample survey of the British population. Sex Transm Infect 2017;93(4):276-83. doi: 10.1136/sextrans-2016-052719

10. Vodstrcil LA, Mclver R, Huston WM, et al. The Epidemiology of Chlamydia trachomatis Organism Load During Genital Infection: A Systematic Review. J Infect Dis 2015;211(10):1628-45. doi: 10.1093/ infdis/jiu670

11. Dirks JA, Wolffs PF, Dukers-Muijrers NH, et al. Chlamydia trachomatis load in population-based screening and STI-clinics: implications for screening policy. PLoS One 2015;10(3):e0121433. doi: 10.1371/journal.pone.0121433

12. Gomes JP, Borrego MJ, Atik B, et al. Correlating Chlamydia trachomatis infectious load with urogenital ecological success and disease pathogenesis. Microbes Infect 2006;8(1):16-26. doi: 10.1016/j.micinf.2005.05.014

13. Agrawal T, Vats V, Salhan S, et al. Determination of chlamydial load and immune parameters in asymptomatic, symptomatic and infertile women. FEMS Immunol Med Microbiol 2009;55(2):250-7. doi: 10.1111/j.1574-695X.2008.00530.x 
14. Walker J, Tabrizi SN, Fairley CK, et al. Chlamydia trachomatis incidence and re-infection among young women--behavioural and microbiological characteristics. PLoS One 2012;7(5):e37778. doi: 10.1371/journal.pone.0037778.

15. van Liere G, Dukers-Muijrers N, Levels L, et al. High Proportion of Anorectal Chlamydia trachomatis and Neisseria gonorrhoeae After Routine Universal Urogenital and Anorectal Screening in Women Visiting the Sexually Transmitted Infection Clinic. Clin Infect Dis 2017;64(12):1705-10. doi: 10.1093/ $\mathrm{cid} / \mathrm{cix} 243$

16. van Liere GA, Hoebe CJ, Wolffs PF, et al. High co-occurrence of anorectal chlamydia with urogenital chlamydia in women visiting an STI clinic revealed by routine universal testing in an observational study; a recommendation towards a better anorectal chlamydia control in women. BMC Infect Dis 2014;14:274. doi: 10.1186/1471-2334-14-274.

17. Wijers J, van Liere G, Hoebe C, et al. Test of cure, retesting and extragenital testing practices for Chlamydia trachomatis and Neisseria gonorrhoeae among general practitioners in different socioeconomic status areas:Aretrospective cohortstudy, 2011-2016. PLoS One 2018;13(3):e0194351. doi: 10.1371/journal.pone.0194351.

18. Bolotin S, Deeks SL, Marchand-Austin A, et al. Correlation of Real Time PCR Cycle Threshold CutOff with Bordetella pertussis Clinical Severity. PLoS One 2015;10(7):e0133209. doi: 10.1371/journal. pone.0133209.

19. Dionne LL, Raymond F, Corbeil J, et al. Correlation between Clostridium difficile bacterial load, commercial real-time PCR cycle thresholds, and results of diagnostic tests based on enzyme immunoassay and cell culture cytotoxicity assay. J Clin Microbio/ 2013;51(11):3624-30. doi: 10.1128/ jcm.01444-13 [published Online First: 2013/08/24].

20. Ott SJ, Musfeldt M, Ullmann U, et al. Quantification of intestinal bacterial populations by real-time PCR with a universal primer set and minor groove binder probes: a global approach to the enteric flora. J Clin Microbio/ 2004;42(6):2566-72. doi: 10.1128/JCM.42.6.2566-2572.2004.

21. Van Bergen JEAM, et al. Standard of the National Society for General Practice: the STI consultation [NHGStandaard: Het soa-consult]. Huisarts en Wetenschap 2013;56(9):450-63.

22. Lunny C, Taylor D, Hoang L, et al. Self-Collected versus Clinician-Collected Sampling for Chlamydia and Gonorrhea Screening: A Systemic Review and Meta-Analysis. PLoS One 2015;10(7):e0132776. doi: 10.1371/journal.pone.0132776.

23. Workowski KA. Centers for Disease Control and Prevention Sexually Transmitted Diseases Treatment Guidelines. Clin Infect Dis 2015;61 Suppl 8:S759-62. doi: 10.1093/cid/civ771.

24. Lanjouw E, Ouburg S, de Vries HJ, et al. 2015 European guideline on the management of Chlamydia trachomatis infections. International journal of STD \& AIDS 2016;27(5):333-48. doi: 10.1177/0956462415618837.

25. van Liere GA, Dirks JA, Hoebe CJ, et al. Anorectal Chlamydia trachomatis Load Is Similar in Men Who Have Sex with Men and Women Reporting Anal Sex. PLoS One 2015;10(8):e0134991. doi: 10.1371/ journal.pone.0134991. 
26. Dirks JA, van Liere GA, Bogers S, et al. Natural Course of Chlamydia trachomatis Bacterial Load in the Time Interval between Screening and Treatment in Anogenital Samples. PLoS One 2015;10(12):e0145693. doi: 10.1371/journal.pone.0145693.

27. Visser M vAF, Op de Coul ELM, Slurink IAL, van Wees DA, Hoenderboom BM, den Daas C, Woestenberg PJ, Götz HM, van Sighem Al, Nielen M, van Benthem BHB. Sexually transmitted infections in the Netherlands in 20172017 [Available from: https://www.rivm.nl/Documenten_en_publicaties/ Wetenschappelijk/Rapporten/2018/Juni/Sexually_transmitted_infections_in_the_Netherlands_ in_2017 accessed 2-7-2018.

28. van der Veer C, van Rooijen MS, Himschoot M, et al. Trichomonas vaginalis and Mycoplasma genitalium: age-specific prevalence and disease burden in men attending a sexually transmitted infections clinic in Amsterdam, the Netherlands. Sex Transm Infect 2016;92(1):83-5. doi: 10.1136/ sextrans-2015-052118.

29. Dubbink JH, de Waaij DJ, Bos M, et al. Microbiological Characteristics of Chlamydia trachomatis and Neisseria gonorrhoeae Infections in South African Women. J Clin Microbiol 2016;54(1):200-3. doi: 10.1128/JCM.02848-15.

30. De Vries H, van Dam A, Bax C. Seksueel Overdraagbare Aandoeningen Multidisciplinaire Richtlijn Update 20182018 [Available from: www.soaaids.nl/nl/professionals/beroepsgroep/arts/ professioneel-handelen/de-specialist accessed 3-9-2018.

31. Kong FY, Tabrizi SN, Fairley CK, et al. The efficacy of azithromycin and doxycycline for the treatment of rectal chlamydia infection: a systematic review and meta-analysis. The Journal of antimicrobial chemotherapy 2015;70(5):1290-7. doi: 10.1093/jac/dku574 [published Online First: 2015/02/01].

32. Dukers-Muijrers N, Wolffs PFG, Vries $\mathrm{H}$, et al. Treatment effectiveness of azithromycin and doxycycline in uncomplicated rectal and vaginal Chlamydia trachomatis infections in women: a multicentre observational study (FemCure). Clin Infect Dis 2019 doi: 10.1093/cid/ciz050.

33. Foschi C, Nardini P, Banzola N, et al. Chlamydia trachomatis infection prevalence and serovar distribution in a high-density urban area in the north of Italy. J Med Microbio/ 2016;65(6):510-20. doi: 10.1099/jmm.0.000261.

34. Mercer CH, Tanton C, Prah P, et al. Changes in sexual attitudes and lifestyles in Britain through the life course and over time: findings from the National Surveys of Sexual Attitudes and Lifestyles (Natsal). Lancet 2013;382(9907):1781-94. doi: 10.1016/S0140-6736(13)62035-8.

35. van Bergen JE, Kerssens JJ, Schellevis FG, et al. Sexually transmitted infection health-care seeking behaviour in the Netherlands: general practitioner attends to the majority of sexually transmitted infection consultations. International journal of STD \& AIDS 2007;18(6):374-9. doi: 10.1258/095646207781024883.

36. Gupta K, Bakshi RK, Van Der Pol B, et al. Repeated Chlamydia trachomatis infections are associated with lower bacterial loads. Epidemiol Infect 2018:1-3. doi: 10.1017/S0950268818002704.

37. Dukers-Muijrers NH, van Liere GA, Hoebe CJ. Re-screening Chlamydia trachomatis positive subjects: a comparison of practices between an STI clinic, general practitioners and gynaecologists. Sex Transm Infect 2013;89(1):25-7. doi: 10.1136/sextrans-2011-050467. 
38. Price MJ, Ades AE, Angelis DD, et al. Mixture-of-exponentials models to explain heterogeneity in studies of the duration of Chlamydia trachomatis infection. Stat Med 2013;32(9):1547-60. doi: 10.1002/sim.5603.

39. Batteiger BE, XU F, Johnson RE, et al. Protective immunity to Chlamydia trachomatis genital infection: evidence from human studies. J Infect Dis 2010;201 Suppl 2:S178-89.

40. Jalal H, Verlander NQ, Kumar N, et al. Genital chlamydial infection: association between clinical features, organism genotype and load. J Med Microbiol 2011;60(Pt 7):881-8. doi: 10.1099/ jmm.0.028076-0.

41. Michel CE, Sonnex C, Carne CA, et al. Chlamydia trachomatis load at matched anatomic sites: implications for screening strategies. J Clin Microbio/ 2007;45(5):1395-402. doi: 10.1128/JCM.00100-07. 


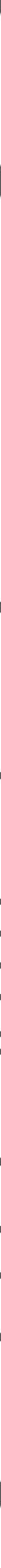





\section{Abstract}

\section{Background}

The Chlamydia trachomatis (CT) bacterial load could have impact on transmission and sequelae. This is the first study to date providing a comparison of the $\mathrm{CT}$ bacterial load of all three anatomic sites estimated by cycle quantification (Cq) values.

\section{Methods}

Laboratory data from 7,900 CT positive samples were included (2012-2018). The Cq value was used as an inversely proportional measure for CT load. Multivariable linear regression analyses were used to assess differences in mean Cq values per sample type.

\section{Results}

Vaginal swabs had the lowest Cq values (31.0) followed by urine samples (32.5), anorectal swabs (34.0) and oropharyngeal swabs (36.8) ( $P<0.001)$. Men and women had similar oropharyngeal (36.4vs.37.3; $\mathrm{p}=0.13$ ) and anorectal (34.2vs.33.9; $\mathrm{P}=0.19)$ Cq values. Men (32.2) and women (30.7) aged $<25$ years had lower urogenital Cq values than men (32.8) and women (31.9) aged $\geq 25$ years $(P<0.001)$. HIV positive patients (33.8) had higher urogenital $C q$ values than HIV negative patients $(32.6)(P<0.03)$.

\section{Conclusions}

Men and women have a similar CT load at extragenital locations arguing for similar transmission potential and clinical relevance. Older patients and patients co-infected with HIV had a lower $\mathrm{CT}$ load suggesting exposure to previous $\mathrm{CT}$ infections potentially leading to some partial immunity reducing $\mathrm{CT}$ load. 


\section{Introduction}

Chlamydia trachomatis (CT) is the most reported bacterial sexually transmitted infection (STI) worldwide. ${ }^{1} \mathrm{CT}$ is associated with reproductive sequelae in women, such as pelvic inflammatory disease (PID), ectopic pregnancy, infertility, and chronic lower abdominal pain. ${ }^{2}$

The CT bacterial load (CT load), frequently expressed as the number of CT bacteria/ml, could potentially affect transmission of the disease and sequelae. ${ }^{34}$ Currently, it is not clear what determines a high CT load in a patient. Although symptoms may be associated with a higher CT load, studies to date show a weak association. ${ }^{356}$ According to a systematic review, only a few nucleic acid amplification test (NAAT)-based studies on associations with CT load have been conducted. ${ }^{35-10}$ Sample sizes of these studies were small, and most studies had a small number of cases within categories of independent determinants. ${ }^{35-10}$ The assessment of determinants for a high CT load could be relevant. For example, viral STIs like human immunodeficiency virus (HIV) and herpes simplex virus have shown increased transmission potential with higher viral load. ${ }^{3}$ Likely the transmission potential of CT is also dependent on CT load. ${ }^{3}$

Extragenital infections, mainly anorectal infections, are common among men who have sex with men (MSM) and women. ${ }^{11}$ Several studies have assessed the CT load of different anatomic locations and sample types. ${ }^{3571213}$ However, those studies used different methods to quantify and report CT load making them less comparable. Therefore, standardization for CT load measurements is needed. ${ }^{14}$ In the current study, we compared the CT load of all relevant urogenital and extragenital sites using the same methods.

Co-infections with Neisseria gonorrhoeae (NG) are prevalent among CT positive high risk individuals such as MSM, young people (aged < 25 years) and some ethnic groups and these co-infections might influence CT load. ${ }^{15-17}$ According to a systematic review, only six studies till date have assessed the association between co-infections and CT load. ${ }^{3}$ One such study observed a higher anorectal CT load among CT positive men co-infected with anorectal NG compared to anorectal NG negative men. ${ }^{18}$ Whereas, another study suggested a lower urogenital CT load among women concurrently infected with CT and NG, however not statistically significant ( $p=0.06) .{ }^{19}$ Associations between CT load and co-infections with other STIs like HIV and syphilis were not observed and it is still unclear due to lack of research evidences. ${ }^{3}$ 121820 Nevertheless, co-infection with other STIs might have impact on acquiring and transmitting CT.

To our knowledge, this is the first and largest study to date providing a comparison of the CT load of all anatomic sites in both men and women estimated by cycle quantification (Cq) values. Our objectives were to assess whether different sample types have a different CT load and whether age and co-infection with NG, HIV, or syphilis were associated with it. 


\section{Methods}

\section{Study population}

Data of CT positive patients (aged $\geq 16$ years) from January 2012 to June 2018 were used in this cross-sectional study. Data derived from 7,224 consultations (with one or more CT positive samples available) originating from 6,170 CT positive patients (from a total of $n=62,306$ CT test consultations; $11.6 \%$ CT positive) were obtained from the laboratory registry of the Medical Microbiology Laboratory of Maastricht University Medical Center (MUMC+). All samples were tested for CT with the same NAAT for both plasmid and chromosomal DNA (COBAS 4800, Roche Diagnostics, Basel, Switzerland), as per the manufacturer's protocol. ${ }^{21}$ We used the NAAT-derived cycle quantification (Cq) value as a proxy for $\mathrm{CT}$ load. The $\mathrm{Cq}$ value reflects the number of amplification cycles that occurred before a positive CT signal was detected. $\mathrm{Cq}$ values show inverse relationships, that is a low $\mathrm{Cq}$ value indicated a high load, and vice versa. $^{22-24}$

Patients were tested on different anatomic locations (urogenital, anorectal and oropharyngeal) according to testing guidelines and indications. ${ }^{25}$ The study included data from all CT positive samples for which the Cq value could be retrieved (99.2\%; 7,900/7,965) (Figure 1). For men, data from first void urine samples (further referred to as urine samples) and urethral swabs were available. Mean Cq values from urine samples ( $n=2,601$, mean=32.49, SD=3.18) and urethral swabs $(n=10$, mean=33.47, $S D=4.89)$ were similar $(p=0.54)$. Urethral swabs of men were excluded. For women, urogenital data were available from urine samples, vaginal swabs, and cervical swabs. The mean Cq values from vaginal swabs ( $n=3,273$, mean=31.00, $\mathrm{SD}=3.72$ ) and cervical swabs ( $\mathrm{n}=147$, mean=30.49, $\mathrm{SD}=3.94)$ were comparable in women $(p=0.11)$. Cervical swabs of women were excluded.

\section{Cycle quantification validation for CT load}

In order to ensure that the Cq value can indeed be used as a proxy for CT load in the different sample types, we compared the Cq values obtained from the COBAS system with previously determined quantitative in-house PCR. ${ }^{12} 2126$ Absolute CT load values (bacteria/ml) were available from a subset of the clinical STI clinic population; 129 urine samples and 69 anorectal swabs from men, and 403 vaginal swabs and 101 anorectal samples from women. In brief, we quantified CT load by an in-house TaqMan real-time PCR to quantify CT OmpA-gene copies/ml. ${ }^{21} \mathrm{~A}$ full description of the CT load quantification has been described elsewhere. ${ }^{21}$

For men, moderate correlations between $\mathrm{Cq}$ values of urine samples and urine CT load (Pearson's r:-0.61, $n=129, p=<0.001$ ), and high correlations between Cq values of anorectal samples and anorectal CT load (Pearson's r:-0.93, $n=69, p=<0.001$ ) were observed. For wom- 
en, high correlations between Cq values of vaginal swabs and vaginal CT load (Pearson's r:-0.88, $n=403, p=<0.001$ ), and high correlations between $\mathrm{Cq}$ values of anorectal swabs and anorectal CT load (Pearson's r:-0.96, $n=101, p=<0.001$ ) were observed. In addition, Cobas Cq values are highly correlated (Pearson's $R^{2}>0.98$ ) with known concentrations of CT (IFU/ml) [personal communication, B. van der Veer, unpublished results]. Therefore, we argue that Cq values are a valid proxy for CT load in urogenital and extragenital samples used in this study.

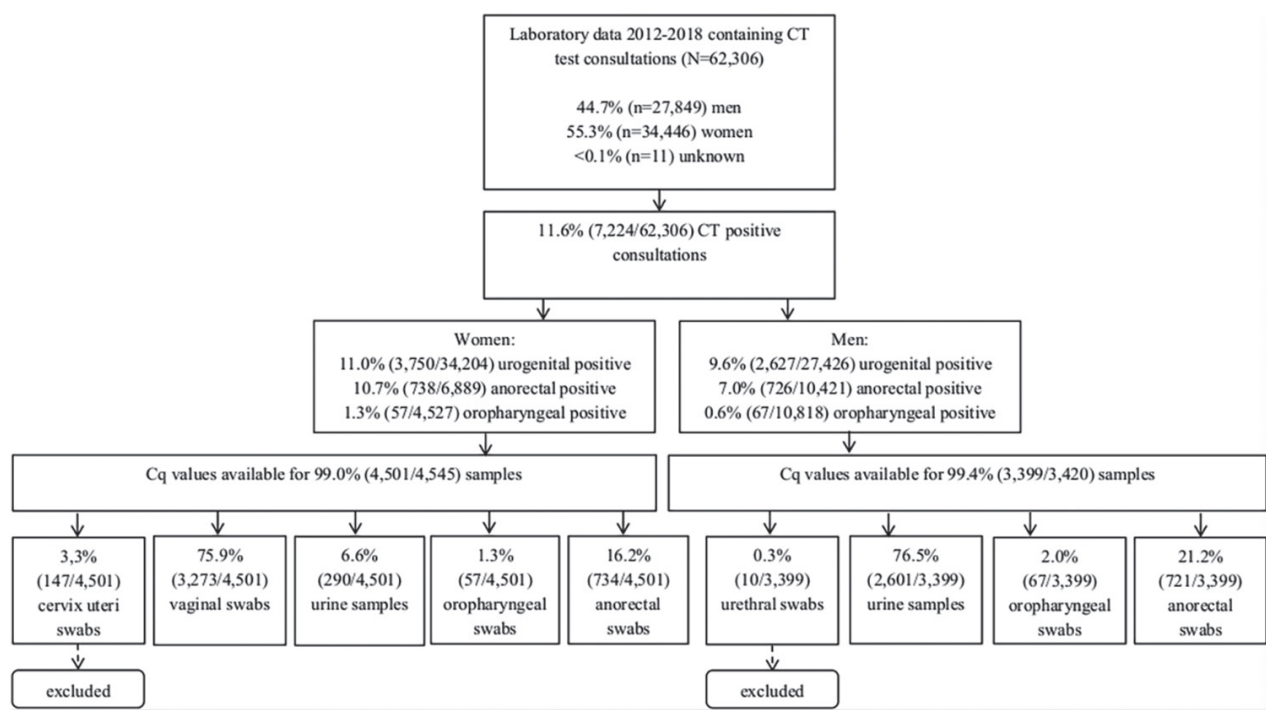

Figure 1. Flowchart, including urogenital and extragenital Chlamydia trachomatis samples of men and women

\section{Statistical analyses}

In the main analyses, the association between "sample type" and the outcome measure "Cq values", as a proxy for CT load, was assessed using univariable and multivariable linear regression analyses adjusting for sex, and age (<25 years, $\geq 25$ years). Anorectal samples were used as the reference group.

In the secondary analyses, association of sex (women, men), age (<25 years, $\geq 25$ years), urogenital NG co-infection, anorectal NG co-infection, oropharyngeal NG co-infection, HIV co-infection, and a positive syphilis screening test (TPHA/TPPA) were assessed with the CT load per sample type, i.e. vaginal swab, urine sample, oropharyngeal swab, and anorectal swab.

In the linear regression analyses, determinants with $p<0.05$ in the univariable model were included in the multivariable model. A p-value of $<0.05$ was considered statistically significant. Mean values, standard deviations, (adjusted) mean differences, and 95\% confidence intervals $(\mathrm{Cl})$ were calculated. Mean differences represented the size of the associations found. 
As Cq values represent values on a log scale, this is a less accessible and incomprehensible measure. To make it more accessible a "factor CT load" that represented 'the number of times the CT load was higher between studied groups', was calculated for the statistically significant determinants using the formula: $2^{\wedge}$ (adjusted mean difference of $C q$ values). For example, the adjusted mean difference of $\mathrm{Cq}$ values between vaginal swabs and anorectal swabs was $-2.87 \mathrm{Cq}$. Therefore, factor load was $2^{\wedge} 2.87=7.3$. Thus, vaginal swabs have a 7.3 times higher CT load compared to anorectal swabs.

All analyses were performed using SPSS Version 24 (IBM SPSS Statistics for Windows, IBM Corporation, Armonk, New York, USA).

\section{Medical Ethical clearance}

The Medical Ethics Committee of the Maastricht University Medical Center (Maastricht, the Netherlands) approved this study (METC 2017-0251) and waived the need for patient consent. Since the retrospective data originated from regular care and were analyzed anonymously, no further informed consent for data analysis was obtained.

\section{Results}

\section{CT load per sample type, estimated by cycle quantification values}

In multivariable analyses, vaginal swabs $(-2.87 \mathrm{Cq})$ and urine samples $(-1.33 \mathrm{Cq})$ had lower Cq values than anorectal swabs, which represented 7.3 and 2.5 times higher $C T$ load, respectively. Oropharyngeal Cq values were higher $(2.73 \mathrm{Cq})$ compared to anorectal swabs, indicating a 6.6 times lower CT load (Table 1).

The range of $\mathrm{Cq}$ values per sample type were comparable for men and women (Figure $2 \mathrm{~A}$ ). Men and women had similar urine $(p=0.15)$, anorectal $(p=0.19)$, and oropharyngeal $(p=0.13)$ $\mathrm{Cq}$ values indicative of similar CT loads (Figure $2 \mathrm{~B}$ ). The mean $\mathrm{Cq}$ values from vaginal swabs $(n=3,273$, mean=31.00, SD=3.7) and urine samples ( $n=290$, mean=32.82, SD=3.7) in women were significantly different ( $p=<0.001$ ). 
Table 1. Main analyses including different sample types and associations with sex and age for Chlamydia trachomatis cycle quantification values as a proxy for CT load.

\section{Cq values}

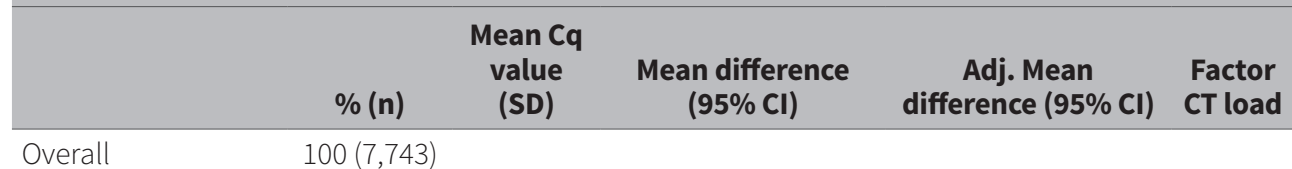

\section{Sample type}

\begin{tabular}{|c|c|c|c|c|}
\hline Vaginal swaba & $42.3(3,273)$ & $31.0(3.7)$ & $-3.02(-3.24$ to -2.80$)$ & $-2.87(-3.13 \text { to }-2.62)^{b}$ \\
\hline Urine sample & $37.3(2,891)$ & $32.5(3.2)$ & $-1.50(-1.74$ to -1.28$)$ & $-1.33(-1.58 \text { to }-1.08)^{c}$ \\
\hline $\begin{array}{r}\text { Oropharyngeal } \\
\text { swab }\end{array}$ & $1.6(124)$ & $36.8(3.1)$ & 2.79 (2.14to 3.44) & $2.73(2.08$ to 3.38$)$ \\
\hline Anorectal swab & $18.8(1,455)$ & $34.0(3.8)$ & Ref & Ref \\
\hline Women & $56.2(4,354)$ & $31.6(4.0)$ & $-1.24(-1.40$ to -1.07$)$ & 0.21 (-2.0.06 to 0.49$)$ \\
\hline Men & $43.8(3,389)$ & $32.9(3.4)$ & Ref & Ref \\
\hline
\end{tabular}

Age $^{\mathrm{e}}$

$$
\begin{array}{cccccc}
<25 \text { years } & 60.3(4,666) & 31.7(3.7) & \mathbf{- 1 . 3 8}(\mathbf{- 1 . 5 5} \text { to } \mathbf{- 1 . 2 1 )}) & \mathbf{- 0 . 7 6}(\mathbf{- 0 . 9 3} \text { to } \mathbf{- 0 . 5 9 )} & 1.7 \\
\geq 25 \text { years } & 39.7(3,077) & 33.1(3.7) & \text { Ref } & \text { Ref } &
\end{array}
$$

a Only measured among women

b When using oropharyngeal samples as the reference group the adjusted mean difference for vaginal swabs was -5.60 (95\% Cl: -6.26 to -4.95$)$, factor CT load: 48.5 .

c When using oropharyngeal samples as the reference group the adjusted mean difference for urine was -4.06 (95\% Cl: -4.70 to -3.41$)$, factor CT load: 16.7.

d Including all sample types, that is vaginal swabs, urine samples, oropharyngeal swabs and anorectal swabs.

e Including all sample types, that is vaginal swabs, urine samples, oropharyngeal swabs and anorectal swabs. Statistically significant associations $(p<0.05)$ are depicted in bold.

Abbreviations: Cq, cycle quantification; Adj., adjusted; Cl, confidence interval; CT, Chlamydia trachomatis; Ref, reference.

\section{Determinants associated with CT load stratified per sample type}

In multivariable analyses, women aged <25 years had lower vaginal Cq values (-0.69 Cq) compared to women aged $\geq 25$ years, resulting in a 1.6 times higher CT load in the former (Table 2 ). HIV positive women had higher vaginal Cq values $(6.86 \mathrm{Cq})$ compared to HIV negative women, which represented an 116.2 times lower CT load.

We additionally assessed whether or not the three HIV infected women were repeatedly infected with CT. All were not repeatedly infected with CT. However, since the analyses included only three HIV infected women this result was unreliable.

In multivariable analyses, patients aged $<25$ years had lower urine $\mathrm{Cq}$ values $(-0.52 \mathrm{Cq})$ compared to patients aged $\geq 25$ years, indicating a 1.4 times higher CT load (Table 2). Patients co-infected with urogenital NG had higher urine $\mathrm{Cq}$ values $(0.96 \mathrm{Cq}$ ) compared to urogenital NG negative patients, representing a 1.9 times lower CT load. HIV positive patients had higher 
urine $\mathrm{Cq}$ values $(0.95 \mathrm{Cq}$ ) compared to HIV negative patients, which represented a 1.9 times lower CT load.

We additionally assessed whether urogenital NG- and HIV co-infected patients with urine samples had repeat CT infections ( $\geq 2$ ) or reported to have an earlier STI (only among STI clinic patients). HIV co-infected patients more often had repeat CT infections compared to HIV negative men (24.4\% vs. 6.4\%; $\mathrm{p}<0.001$ ). Patients co-infected with HIV reported to have had an earlier STI more often compared to HIV negative men (35.7\% vs. 16.0\%; $p<0.001$ ). Patients co-infected with NG had similar repeat CT infections compared to urogenital NG negative patients (11.2\% vs. 9.1\%; $p=0.69)$. Patients co-infected with NG frequently reported to have had an earlier STI compared to NG negative patients (31.7\% vs. 19.5\%; $p=0.07$ ).

No determinants were associated with oropharyngeal and anorectal CT load in multivariable analyses (see Supplementary Table 1). 


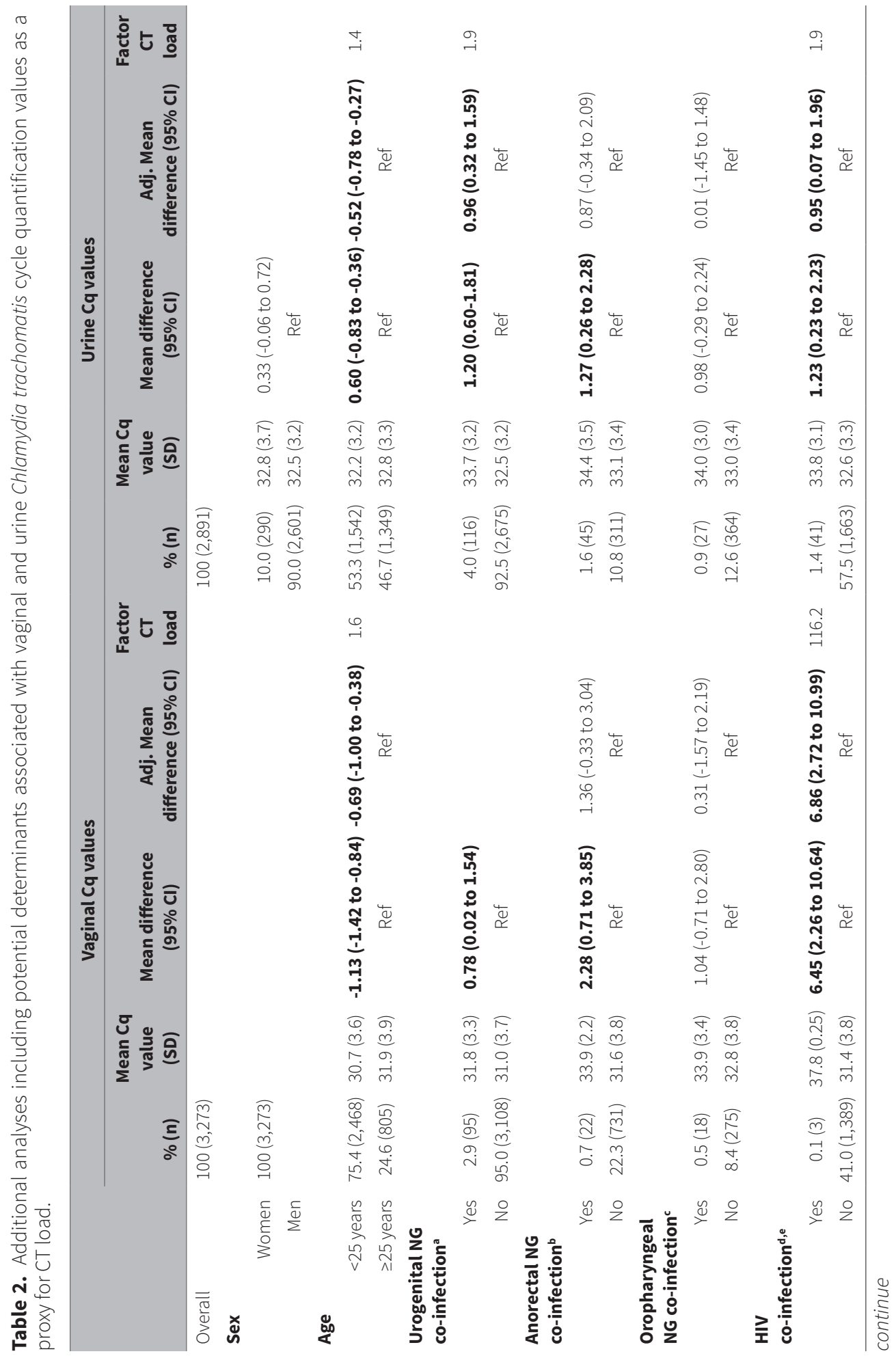


용

온

乐

ฟิ

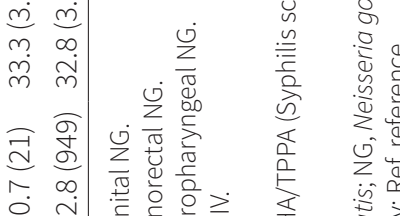




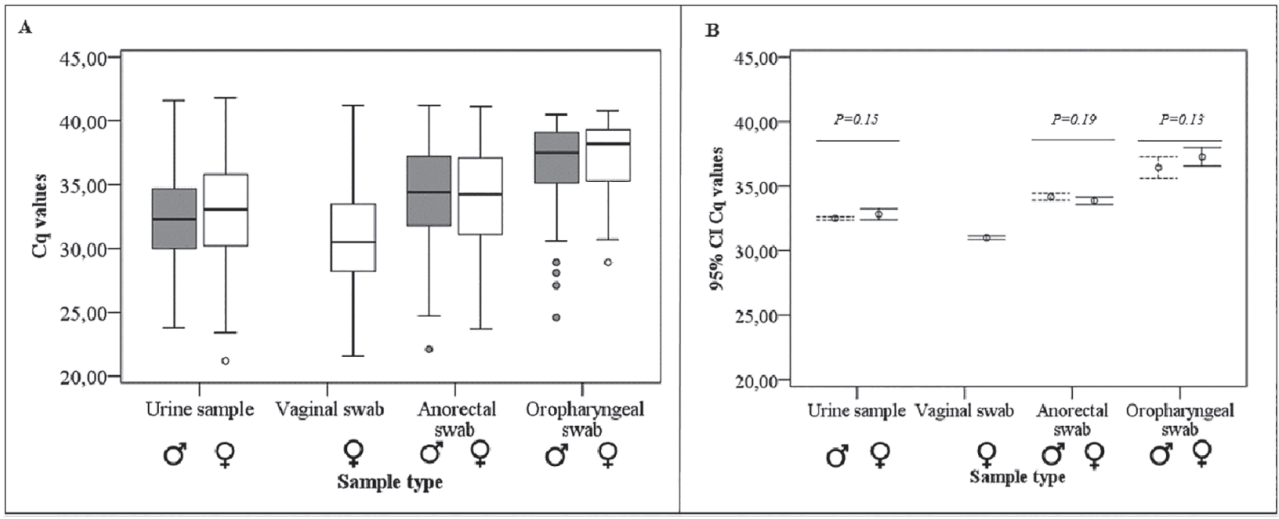

Figure 2. Boxplots and error bars showing the distribution of Cq values per sample type for men and women.

(A) Boxplots showing the distribution of Cq values, as a proxy for CT load, per sample type for men (in grey) and women (in white). Outliers are indicated by circles. (B) Error bars showing mean $\mathrm{Cq}$ values and 95\% confidence intervals, as a proxy for CT load, per sample type for men (dotted line) and women (solid line). Univariable regression analyses were performed to test unadjusted mean Cq value differences between men and women. Men and women had similar urine (mean=32.4, SD=3.2 versus mean=32.8, $S D=3.7, P=0.15)$, oropharyngeal (mean=36.4, $S D=3.4$ versus mean=37.3, $S D=2.7, P=0.13$ ) and anorectal $($ mean $=34.2, \mathrm{SD}=3.6$ versus mean $=33.9, \mathrm{SD}=3.9, \mathrm{P}=0.19) \mathrm{Cq}$ values.

\section{Discussion}

This is the first and largest study to date providing a comparison of CT load, estimated by Cq values, in urogenital and extragenital locations of men and women. The mean CT load differed significantly per sample type and subsequently per anatomic location. Notably, no difference ( $p>0.05$ ) in CT load was observed for men and women on extragenital locations arguing for similar transmission potential, clinical relevance and NAAT detection limits. Moreover, vaginal swabs had a higher CT load compared to urine samples confirming the preference for vaginal swabs above urine samples in women. A higher urogenital CT load was observed in younger patients, whereas a lower urogenital CT load was found in patients co-infected with HIV and NG.

The strength of the current study is the comparison of CT load of different sample types of both men and women in one large study using the same diagnostic test (NAAT) allowing for the best comparison possible. CT load can be quite variable in different patients depending on different factors, including time of diagnosis since acquiring the infection. However, our study group showed that the majority of patients had a stable CT load in the time interval between diagnosis and treatment $(66.3 \%, 73.1 \%$ and $48.6 \%$ for vaginal swabs, urine samples and anorectal swabs respectively. ${ }^{26}$ The $\mathrm{CT}$ load for each sample type might also vary as patients could apply the sampling instructions differently. Nevertheless, mean CT load on population level was quite stable as seen in our results of anorectal and oropharyngeal 
samples across both sexes. ${ }^{6}$ In earlier work we already discussed the limitations of comparing different sampling methods (i.e. urine versus swabs) and different sampling locations (vaginal swab versus anorectal or oropharyngeal swabs). For example, different sites have different epithelial cells, which might influence the CT load. Some previous studies included the number of cells into the CT load count but as anatomical samples sites differ in number of cells in the samples, we argue that this hampers good comparison. ${ }^{14}$ The Cq values of urine samples of men were moderately, but significantly, correlated with urine CT load (expressed as copies/ml). Therefore, the results of urine $\mathrm{Cq}$ values could be a less stable indication for CT load. This is an expected finding as we know concentration in urine can differ inter- and intra-individually and with time since infected cells from the male urethra may get washed out by first void urine. Another reason could be that the CT major outer membrane protein (MOMP) break down faster in urine samples than in vaginal swabs leading to higher Cq values in the in-house qPCR (Taqman) compared to lower Cq values in the NAAT (Cobas 4800). Furthermore, we were unable to assess whether the amplified DNA was from viable CT or non-viable CT. Earlier our study group developed a new viability technique (viability-pcr) to discriminate between viable and non-viable CT and showed that a substantial amount of CT DNA originated from non-viable cells. ${ }^{27}$ The non-viable CT DNA would have no impact on CT transmission and sequelae.

There is an ongoing discussion about what difference in CT load is clinically or microbiologically relevant. Earlier, our group used a difference of 1 log load (3.3 Cq) as microbiology relevant to overcome potential technical variations when measuring the $\mathrm{CT}$ load within the same patient over time. However, in the current study, we averaged the Cq values over an entire group (for example: men, women, or patients aged <25 year). Therefore, potential technical differences between individuals are flattened out, and have little impact on the results since these variations are randomly distributed over all samples tested within this particular group. ${ }^{26}$ The precise clinical or microbiological relevance of the differences in CT load between groups remains unknown. However, the fact that men and women had a similar extragenital CT load indicate similar clinical relevance.

Nevertheless, of all sample types included, vaginal swabs had the highest CT load, which could relate to the highest impact on transmission. Recently, our group showed a borderline significant association ( $\mathrm{P}=0.054$ ) between a higher urogenital $\mathrm{CT}$ load and frequent microbiological CT detection in women after treatment with azithromycin compared to vaginal swabs with a low CT load. ${ }^{28}$ Our current study confirmed the high urogenital CT load, which might be clinically relevant when it hampers the treatment of a CT infection with azithromycin.

Our research team and others have shown that the majority of women (summary estimate of 68.1\%) diagnosed with urogenital CT also have a concurrent anorectal CT infection irrespective of reported anal sex or anal symptoms. ${ }^{29} 30$ The clinical relevance (risk of complications) and public health implications (transmission potential) associated with anorectal CT detection in women are, however, under debate. ${ }^{31}$ Anorectal infections in women could result from autoinoculation due to the close proximity between the vagina and anorectum. ${ }^{1932}$ 
In a study conducted by our researchers, a sample ( $\mathrm{n}=105)$ of concurrently infected STI clinic women showed that in the majority of cases (in $79 \%$ and $56 \%$ of women reporting anorectal intercourse and no anorectal intercourse, respectively), the anorectal CT load was in the same range as the urogenital CT load, suggesting similar clinical relevance. ${ }^{33}$ Moreover, in the current study, we confirmed this finding and also showed that the anorectal CT load in men equals that of women. We saw as many low as high anorectal loads in both men and women as suggested in an earlier smaller study based on CT qPCR. ${ }^{12}$ While diagnosis and treatment for anorectal CT in MSM is possible, diagnosis and treatment of anorectal CT in women is still under debate. This is in contrast with the fact that a similar load should suggest a similar clinical relevance. Relevance in women might be even more substantial as they bear the burden of reproductive morbidity caused by CT infections. ${ }^{2}$ Moreover, women concurrently infected with urogenital and anorectal CT have a 8.5 times higher urogenital CT load compared to anorectal negative women. ${ }^{6}$ Therefore, women concurrently infected with urogenital and anorectal CT might have higher chances of sequelae such as reproductive morbidity. Although, anorectal CT infections in women might be coincidently treated with a urogenital infections due to the high concurrency, a recent study from our research group suggested that frequently used azithromycin may be less effective than doxycycline in clearing anorectal CT infections in women. ${ }^{28}$ Nevertheless, the question remains whether and how substantial auto-inoculation or other mechanisms for transmission from the female anorectal site to the female urogenital site occur and vice versa.

Studies have shown a lower CT load among older patients and patients with repeat CT infections potentially due to development of partial immunity against CT. ${ }^{893435}$ Especially in HIV infected men (high risk group) who were repeatedly infected with CT, development of partial immunity against CT might explain a lower CT load.

In conclusion, the mean CT load estimated by $\mathrm{Cq}$ values differed per sample type and subsequently per anatomic location. Vaginal swabs had the highest CT load, whereas oropharyngeal swabs had the lowest CT load. Notably, men and women had a similar CT load on extragenital locations arguing similar transmission potential and clinical relevance. A lower CT load was associated with older age (>25) and co-infections with NG and HIV, which was suggestive of exposure to previous CT and potential development of some kind of partial immunity reducing CT load. 


\section{References}

1. Newman L, Rowley J, Vander Hoorn S, et al. Global Estimates of the Prevalence and Incidence of Four Curable Sexually Transmitted Infections in 2012 Based on Systematic Review and Global Reporting. PLoS One 2015;10(12):e0143304. doi: 10.1371/journal.pone.0143304.

2. Haggerty CL, Gottlieb SL, Taylor BD, et al. Risk of sequelae after Chlamydia trachomatis genital infection in women. J Infect Dis 2010;201 Suppl 2:S134-55. doi: 10.1086/652395.

3. Vodstrcil LA, Mclver R, Huston WM, et al. The Epidemiology of Chlamydia trachomatis Organism Load During Genital Infection: A Systematic Review. J Infect Dis 2015;211(10):1628-45. doi: 10.1093/ infdis/jiu670.

4. Rogers SM, Miller WC, Turner CF, et al. Concordance of chlamydia trachomatis infections within sexual partnerships. Sex Transm Infect 2008;84(1):23-8. doi: 10.1136/sti.2007.027029.

5. Jalal H, Verlander NQ, Kumar N, et al. Genital chlamydial infection: association between clinical features, organism genotype and load. J Med Microbiol 2011;60(Pt 7):881-8. doi: 10.1099/ jmm.0.028076-0.

6. Wijers J, Hoebe C, van Liere G, et al. Chlamydia trachomatis bacterial load, estimated by Cq values, in urogenital samples from men and women visiting the general practice, hospital or STI clinic. PLoS One 2019;14(4):e0215606. doi: 10.1371/journal.pone.0215606.

7. Michel CE, Sonnex C, Carne CA, et al. Chlamydia trachomatis load at matched anatomic sites: implications for screening strategies. J Clin Microbiol 2007;45(5):1395-402. doi: 10.1128/ JCM.00100-07.

8. Gomes JP, Borrego MJ, Atik B, et al. Correlating Chlamydia trachomatis infectious load with urogenital ecological success and disease pathogenesis. Microbes Infect 2006;8(1):16-26. doi: 10.1016/j.micinf.2005.05.014.

9. Wiggins R, Graf S, Low N, et al. Real-time quantitative PCR to determine chlamydial load in men and women in a community setting. J Clin Microbio/ 2009;47(6):1824-9. doi: 10.1128/JCM.00005-09.

10. Walker J, Fairley CK, Bradshaw CS, et al. 'The difference in determinants of Chlamydia trachomatis and Mycoplasma genitalium in a sample of young Australian women'. BMC Infect Dis 2011;11:35. doi: 10.1186/1471-2334-11-35.

11. Chan PA, Robinette A, Montgomery M, et al. Extragenital Infections Caused by Chlamydia trachomatis and Neisseria gonorrhoeae: A Review of the Literature. Infect Dis Obstet Gynecol 2016;2016:5758387. doi: 10.1155/2016/5758387.

12. van Liere GA, Dirks JA, Hoebe CJ, et al. Anorectal Chlamydia trachomatis Load Is Similar in Men Who Have Sex with Men and Women Reporting Anal Sex. PLoS One 2015;10(8):e0134991. doi: 10.1371/ journal.pone.0134991.

13. van Rooijen MS, van der Loeff MF, Morre SA, et al. Spontaneous pharyngeal Chlamydia trachomatis RNA clearance. A cross-sectional study followed by a cohort study of untreated STI clinic patients in Amsterdam, The Netherlands. Sex Transm Infect 2015;91(3):157-64. doi: 10.1136/ sextrans-2014-051633. 
14. Dirks JAMC, Hoebe CJPA, van Liere GAFS, et al. Standardisation is necessary in urogenital and extragenital <em>Chlamydia trachomatis</em> bacterial load determination by quantitative PCR: a review of literature and retrospective study. Sexually Transmitted Infections 2019:sextrans-2018-053522. doi: 10.1136/sextrans-2018-053522.

15. vanVeen MG, KoedijkFD, van derSandeMA, etal. STD coinfections in The Netherlands: Specific sexual networks at highest risk. Sex Transm Dis 2010;37(7):416-22. doi: 10.1097/OLQ.0b013e3181cfcb34.

16. Tongtoyai J, Todd CS, Chonwattana W, et al. Prevalence and Correlates of Chlamydia trachomatis and Neisseria gonorrhoeae by Anatomic Site Among Urban Thai Men Who Have Sex With Men. Sex Transm Dis 2015;42(8):440-9. doi: 10.1097/OLQ.0000000000000311.

17. Lim RB, Wong ML, Cook AR, et al. Determinants of Chlamydia, Gonorrhea, and Coinfection in Heterosexual Adolescents Attending the National Public Sexually Transmitted Infection Clinic in Singapore. Sex Transm Dis 2015;42(8):450-6. doi: 10.1097/OLQ.0000000000000316.

18. Boisvert JF, Koutsky LA, Suchland RJ, et al. Clinical features of Chlamydia trachomatis rectal infection by serovar among homosexually active men. Sex Transm Dis 1999;26(7):392-8.

19. Dubbink JH, de Waaij DJ, Bos M, et al. Microbiological Characteristics of Chlamydia trachomatis and Neisseria gonorrhoeae Infections in South African Women. J Clin Microbiol 2016;54(1):200-3. doi: 10.1128/JCM.02848-15.

20. Barnes RC, Katz BP, Rolfs RT, et al. Quantitative culture of endocervical Chlamydia trachomatis. J Clin Microbiol 1990;28(4):774-80.

21. Dirks JA, Wolffs PF, Dukers-Muijrers NH, et al. Chlamydia trachomatis load in population-based screening and STI-clinics: implications for screening policy. PLoS One 2015;10(3):e0121433. doi: 10.1371/journal.pone.0121433.

22. Bolotin S, Deeks SL, Marchand-Austin A, et al. Correlation of Real Time PCR Cycle Threshold CutOff with Bordetella pertussis Clinical Severity. PLoS One 2015;10(7):e0133209. doi: 10.1371/journal. pone.0133209.

23. Dionne LL, Raymond F, Corbeil J, et al. Correlation between Clostridium difficile bacterial load, commercial real-time PCR cycle thresholds, and results of diagnostic tests based on enzyme immunoassay and cell culture cytotoxicity assay. J Clin Microbio/ 2013;51(11):3624-30. doi: 10.1128/ jcm.01444-13 [published Online First: 2013/08/24].

24. Ott SJ, Musfeldt M, Ullmann U, et al. Quantification of intestinal bacterial populations by real-time PCR with a universal primer set and minor groove binder probes: a global approach to the enteric flora. J Clin Microbiol 2004;42(6):2566-72. doi: 10.1128/JCM.42.6.2566-2572.2004.

25. De Vries H, van Dam A, Bax C. Seksueel Overdraagbare Aandoeningen Multidisciplinaire Richtlijn Update 20182018 [Available from: www.soaaids.nl/nl/professionals/beroepsgroep/arts/ professioneel-handelen/de-specialist accessed 3-9-2018.

26. Dirks JA, van Liere GA, Bogers S, et al. Natural Course of Chlamydia trachomatis Bacterial Load in the Time Interval between Screening and Treatment in Anogenital Samples. PLoS One 2015;10(12):e0145693. doi: 10.1371/journal.pone.0145693. 
27. Janssen KJ, Hoebe CJ, Dukers-Muijrers NH, et al. Viability-PCR Shows That NAAT Detects a High Proportion of DNA from Non-Viable Chlamydia trachomatis. PLoS One 2016;11(11):e0165920. doi: 10.1371/journal.pone.0165920.

28. Dukers-Muijrers N, Wolffs PFG, Vries $\mathrm{H}$, et al. Treatment effectiveness of azithromycin and doxycycline in uncomplicated rectal and vaginal Chlamydia trachomatis infections in women: a multicentre observational study (FemCure). Clin Infect Dis 2019 doi: 10.1093/cid/ciz050.

29. van Liere G, Dukers-Muijrers N, Levels L, et al. High Proportion of Anorectal Chlamydia trachomatis and Neisseria gonorrhoeae After Routine Universal Urogenital and Anorectal Screening in Women Visiting the Sexually Transmitted Infection Clinic. Clin Infect Dis 2017;64(12):1705-10. doi: 10.1093/ $\mathrm{cid} / \mathrm{cix} 243$.

30. Chandra NL, Broad C, Folkard K, et al. Detection of Chlamydia trachomatis in rectal specimens in women and its association with anal intercourse: a systematic review and meta-analysis. Sex Transm Infect 2018;94(5):320-26. doi: 10.1136/sextrans-2017-053161.

31. Ding A, Challenor R. Rectal Chlamydia in heterosexual women: more questions than answers. International journal of STD \& AIDS 2014;25(8):587-92. doi: 10.1177/0956462413515637.

32. Heijne JCM, van Liere GAFS, Hoebe CJPA, et al. What explains anorectal chlamydia infection in women? Implications of a mathematical model for test and treatment strategies. Sexually transmitted infections 2017;93(4):270-75. doi: 10.1136/sextrans-2016-052786 [published Online First: 12/16].

33. Dirks J, van Liere G, Hoebe C, et al. Genital and anal Chlamydia trachomatis bacterial load in concurrently infected women: a cross-sectional study. Sex Transm Infect 2019 doi: 10.1136/ sextrans-2018-053678 [published Online First: 2019/05/18].

34. Gupta K, Bakshi RK, Van Der Pol B, et al. Repeated Chlamydia trachomatis infections are associated with lower bacterial loads. Epidemiol Infect 2018:1-3. doi: 10.1017/S0950268818002704.

35. Walker J, Tabrizi SN, Fairley CK, et al. Chlamydia trachomatis incidence and re-infection among young women--behavioural and microbiological characteristics. PLoS One 2012;7(5):e37778. doi: 10.1371/journal.pone.0037778. 



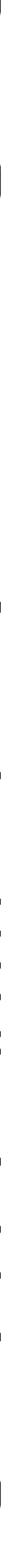





\section{Abstract}

We assessed whether patients repeatedly infected with Chlamydia trachomatis (CT) have a lower urogenital or anorectal CT load. A CT positive retest was independently associated with higher vaginal- and higher urine $\mathrm{Cq}$-values $(\mathrm{p} \leq 0.001)$. Partial immunity could play a role in repeat urogenital infections, potentially not in anorectal infections. 


\section{Introduction}

Repeat Chlamydia trachomatis (CT) infections remain a challenge in CT control. Repeat CT infections can be caused by reinfections from an untreated sexual partner, new infection from the regular or new partner or from a persistent infection due to treatment failure. Repeat CT infections are common; up to 32\% of patients with CT infection test positive again within 1 year of diagnosis $(1,2)$. Repeat infections increase the risk of developing serious reproductive sequelae (3). Therefore, retesting CT-positive patients within 3 to 12 months after initial test is an effective control strategy and can be used to enhance the population-based prevention of CT (4). Although retesting is advised in international guidelines, retesting rates remain typically low (15-40\%) (4).

A high CT bacterial load (hereafter, CT load), frequently expressed as the number of CT bacteria/mL, could potentially affect transmission and occurrence of sequelae (5). Some studies suggest that repeat CT infections are associated with a lower CT load potentially due to partial immunity against CT $(6,7)$. Nevertheless, these studies on CT load and repeat infections had some limitations like including only small numbers of patients, presenting only univariable analyses, or only assessing the urogenital site of women $(6,7)$. Furthermore, data on repeat anorectal infections are lacking although these infections are common among both men and women who have sex with men (MSM) (8). Therefore, we studied CT load in a relatively large number of both repeat positive urogenital and anorectal samples from men and women.

\section{Material and methods}

\section{Study population}

Data from 6,170 CT positive patients (aged $\geq 16$ years) with 7,224 CT positive consultations from January 2012 to June 2018 were used to derive our study population of repeat positives in both men and women from urogenital and anorectal samples. This data originated from the laboratory registry of the Medical Microbiology Laboratory of Maastricht University Medical Center (MUMC+) from a total of 62,306 CT tests with a CT positivity of $11.6 \%$ (9).

All samples were tested for CT with the same NAAT for both plasmid and chromosomal DNA (COBAS 4800, Roche Diagnostics, Basel, Switzerland), as per the manufacturer's protocol (10). We used the NAAT-derived Cq value as a proxy for CT load (9).

For women, CT positive vaginal swabs and CT positive anorectal swabs were included. For men, CT positive first void urine samples and CT positive anorectal swabs were included. The first positive CT test of a patient was defined as the "initial positive test". Only patients with at least one CT positive retest were included in the study population. Samples which tested 
positive for CT within 30 days of a previous positive CT sample were excluded to rule out positivity due to possible false-positive results of non-viable recurrent CT ( $n=230$ vaginal swabs, $\mathrm{n}=76$ urine samples, and $\mathrm{n}=66$ anorectal swabs). All positive samples taken later than 30 days after the initial positive test were considered as a "positive retest" in the entire study period.

In total, $n=429$ patients with at least one urogenital or anorectal CT positive retest were included. Of this sample, $51.5 \%$ were men, the mean age was 28 years, and the vast majority had a western ethnicity (77.2\%). Furthermore, 88.1\% were diagnosed at the STI clinic, 9.3\% by the general practitioner, and $2.6 \%$ by hospital medical specialists. Of the patients diagnosed at the STI clinic, 26.4\% were heterosexual men, 26.6\% were men who have sex with men (MSM), and $47.0 \%$ were women.

Of the 137 men with at least one positive urogenital CT retest, 127 had one positive urine retest, and 10 had two positive retests (total: $n=147$ ). Of the 84 men with at least one positive anorectal CT retest, 63 had one positive retest, 16 had two positive retests, and 5 had three positive retests (total: $\mathrm{n}=110$ ).

Of the 187 women with at least one positive vaginal CT retest, 170 had one positive retest, 16 women had two positive retests, and 1 woman had three positive retests (total: $n=205$ ). Of the 21 women with at least one positive anorectal CT retest, 19 had one positive retest, 1 had two positive retests, and 1 woman had three positive retests (total: $n=24$ ).

\section{Cycle quantification validation for CT load}

We compared the Cq values obtained from the diagnostic NAAT with previously determined quantitative in-house PCR, to ensure that the $\mathrm{Cq}$ value can indeed be used as a proxy for CT load in the different sample types (10-12). In brief, we quantified CT load by an in-house TaqMan real-time PCR to quantify CT OmpA-gene copies/ml (10). A full description of the CT load quantification has been described elsewhere (10). In an earlier publication we described the correlations between Cq values and CT load per sample type in detail (9). Overall, high correlations were observed (Pearson's r: >0.88), except for urine samples (Pearson's r: 0.61) (9).

\section{Statistical analyses}

The main determinant tested was "positive retest" (binary), in which the initial positive test was used as the reference group and any subsequent positive CT test as the group of interest per sample type. In the main analyses, the association between "positive retest" and the outcome measure "Cq values", as a proxy for CT load, was assessed using generalized linear models for repeated measures per sample type (vaginal swab, urine sample and anorectal swab). The analyses dataset was aggregated on a consultation level. Every subsequent CT positive consultation of the same individual, later than at least 30 days of a previous consultation, was 
counted as a "positive retest" when the same sample type as at the initial consultation was taken. We adjusted for gender, age (continuous), year of consultation (continuous), number of days between subsequent CT infection (continuous), concurrent anorectal CT, concurrent oropharyngeal CT, co-infection with urogenital NG, coinfection with anorectal NG, coinfection with oropharyngeal NG and co-infection with HIV. Only multivariable results are presented. A p-value of $<0.05$ was considered statistically significant. Mean values, standard deviations, (adjusted) mean differences, and 95\% confidence intervals (CI) were calculated.

All analyses were performed using SPSS Version 24 (IBM SPSS Statistics for Windows, IBM Corporation, Armonk, New York, USA).

\section{Medical ethical clearance}

The Medical Ethics Committee of the Maastricht University Medical Center (Maastricht, the Netherlands) approved this study (METC 2017-0251) and waived the need for individual written patient consent. Since the retrospective data originated from regular care and were analyzed anonymously, no further informed consent for data analysis was obtained.

\section{Results}

\section{Men}

In multivariable analyses, a positive urine retest ( $\mathrm{n}=147$ ) was associated with higher $\mathrm{Cq}$ values (mean difference $1.08 \mathrm{Cq}$; 95\% Cl 0.27 to 1.90; $p=0.009$ ) compared to the initial positive urine test ( $n=137$ ) (Figure 1 ). In a sensitivity analyses, by including only the first positive urine retest, results were similar (mean difference $1.00 \mathrm{Cq} ; 95 \% \mathrm{Cl} 0.17$ to $1.82 ; \mathrm{p}=0.02$ )

In multivariable analyses, a positive anorectal retest $(\mathrm{n}=110)$ was not associated with $\mathrm{Cq}$ values (mean difference $0.10 \mathrm{Cq}$; $95 \% \mathrm{Cl}-1.15$ to 1.35 ; $\mathrm{p}=0.88$ ) (Figure 1 ). In a sensitivity analyses, by including only the first positive anorectal retest, results were similar and not statistically significant.

\section{Women}

In multivariable analyses, a CT positive vaginal retest ( $\mathrm{n}=205$ ) was associated with higher vaginal Cq values compared to the initial positive vaginal test ( $\mathrm{n}=187$ ) (mean difference $1.51 \mathrm{Cq}$; 95\% Cl 0.58 to 2.44; $p=0.002$ ) (Figure 1 ). In a sensitivity analyses, by including only the first 
positive vaginal retest, results were similar (mean difference $1.36 \mathrm{Cq}$; $95 \% \mathrm{Cl} 0.42$ to 2.30; $p=0.004)$

In multivariable analyses, a CT positive anorectal retest ( $n=24$ ) was not associated with $\mathrm{Cq}$ values in women (mean difference $1.00 \mathrm{Cq}$; $95 \% \mathrm{Cl}-1.87$ to 3.87; $\mathrm{p}=0.49$ ) (Figure 1). In a sensitivity analyses, by including only the first positive anorectal retest, results were similar and not statistically significant.

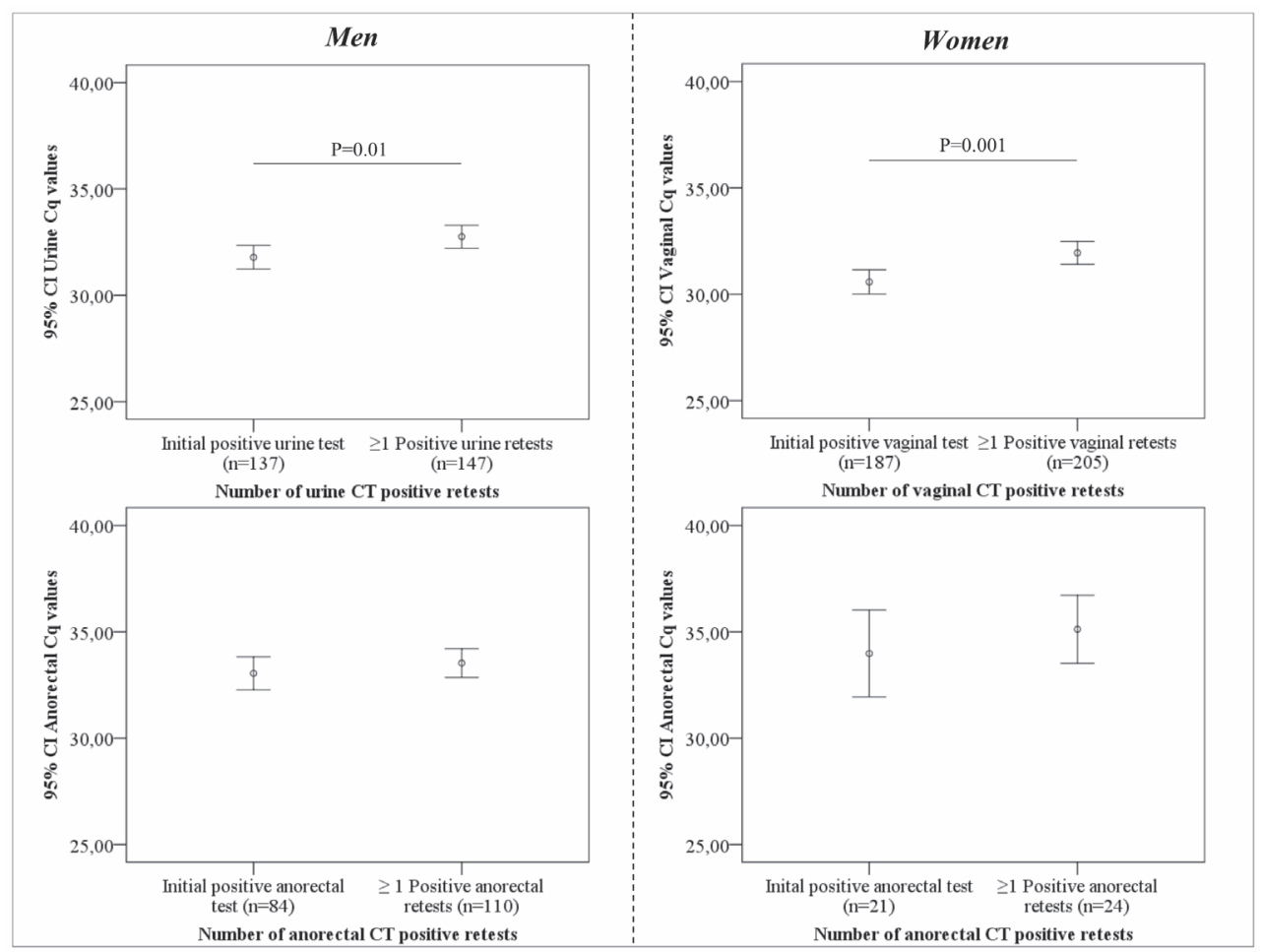

Figure 1. Errors bars with $95 \%$ confidence intervals showing the Chlamydia trachomatis bacterial load, estimated by $\mathrm{Cq}$ values, of the initial positive test and $\geq 1$ repeat infections of the urogenital and anorectal site of men and women. 


\section{Discussion}

This is the largest study to date assessing the CT bacterial load, estimated by Cq values, of repeat $\mathrm{CT}$ infections per sample type adjusted for potential confounders. Men and women repeatedly infected with CT have higher urogenital $\mathrm{Cq}$ values and likely a lower CT load. However, this was not true for anorectal samples as no difference was observed in Cq values in anorectal samples of both men and women.

A strength of our study is the relatively large number of repeat infections analyzed stratified per sample type. Our multivariable analyses confirm the univariable findings of smaller studies indicating that women repeatedly infected with CT have a lower urogenital CT load $(6,7)$. To our knowledge, this is the first study showing that also men repeatedly infected with CT have a lower urogenital CT load.

A limitation was that the $\mathrm{Cq}$ values of urine samples of men were moderately correlated with the CT load of urine samples of men (Pearson's r: 0.61). Therefore, the results of urine Cq values could be a less stable indication of CT load. This is an expected finding as we know concentration in urine can differ inter- and intra-individually and with time because infected cells from the male urethra may get washed out by first void urine.

We showed that men and women repeatedly infected with CT have a lower urogenital CT load, which was also observed in earlier studies, but only among women(6, 7). Both studies suggest that partial immunity against CT could play a role. However, further research is needed including longitudinal data, immunological markers, and duration of the CT infection for more evidence. Furthermore, patients with a lower CT load could have a lower impact on transmission of CT. Likely patients with initial CT infections may have a higher impact on ongoing transmission than patients repeatedly infected with CT. This could highlight the importance of targeting control measures, such as retesting, to those patients with an initial CT infection.

Repeat anorectal CT infections of women were not associated with a lower CT load, which could be a result of the relatively low number of anorectal repeat infections diagnosed among women ( $n=24)$. Among men, however, a substantial number of repeat anorectal infections were diagnosed $(n=110)$ and there was also no association between CT load and repeat infection. If the number of repeat anorectal CT infections in women were larger, we expect a similar anorectal load of repeat infections as men, since men and women have an equal extragenital load (9). Nevertheless, as anorectal infections are common among MSM and women, testing, and retesting the anorectal site remains important to enhance CT control. Potentially the partial immunity theory does not hold for anorectal infections, which could be related to treatment (azithromycin vs. doxycycline). As the treatment effectiveness of doxycycline is higher than azithromycin in uncomplicated anorectal infections (13), it could potentially affect the immune response of the body. However, this is only speculative and further research is needed to assess this pattern. 
An earlier study assessed the CT load of repeat infections among African American women and showed a lower urogenital CT load when repeatedly infected with CT (5.6 CT/ml vs. 4.5 $\mathrm{CT} / \mathrm{ml} ; \mathrm{P}=0.015)$ (6). The authors suggest that the generalizability of the results could be a problem for other populations. However, our results among mainly western individuals are comparable to this particular study.

In conclusion, men and women repeatedly infected with CT have a lower urogenital bacterial load potentially due to partial immunity against CT. However, repeat anorectal infections of men and women were not associated with a lower CT load indicating repeat anorectal infections have a similar impact on transmission and sequalae as initial anorectal infections. 


\section{References}

1. Hosenfeld CB, Workowski KA, Berman S, et al. Repeat infection with Chlamydia and gonorrhea among females: a systematic review of the literature. Sex Transm Dis. 2009;36(8):478-89.

2. Fung M, Scott KC, Kent CK, Klausner JD. Chlamydial and gonococcal reinfection among men: a systematic review of data to evaluate the need for retesting. Sex Transm Infect. 2007;83(4):304-9.

3. den Heijer CDJ, Hoebe C, Driessen JHM, et al. Chlamydia trachomatis and the Risk of Pelvic Inflammatory Disease, Ectopic Pregnancy, and Female Infertility: A Retrospective Cohort Study Among Primary Care Patients. Clin Infect Dis. 2019;69(9):1517-25.

4. Workowski KA. Centers for Disease Control and Prevention Sexually Transmitted Diseases Treatment Guidelines. Clin Infect Dis. 2015;61 Suppl 8:S759-62.

5. Vodstrcil LA, Mclver R, Huston WM, Tabrizi SN, Timms P, Hocking JS. The Epidemiology of Chlamydia trachomatis Organism Load During Genital Infection: A Systematic Review. J Infect Dis. 2015;211(10):1628-45.

6. Gupta K, Bakshi RK, Van Der Pol B, et al. Repeated Chlamydia trachomatis infections are associated with lower bacterial loads. Epidemiol Infect. 2018:1-3.

7. Walker J, Tabrizi SN, Fairley CK, et al. Chlamydia trachomatis incidence and re-infection among young women--behavioural and microbiological characteristics. PLoS One. 2012;7(5):e37778.

8. Chan PA, Robinette A, Montgomery M, et al. Extragenital Infections Caused by Chlamydia trachomatis and Neisseria gonorrhoeae: A Review of the Literature. Infect Dis Obstet Gynecol. 2016;2016:5758387.

9. Wijers J, Dukers-Muijrers N, van Liere G, Dirks J, Wolffs PFG, Hoebe C. Men and women have an equal oropharyngeal and anorectal Chlamydia trachomatis bacterial load: a comparison of three anatomic sites. J Infect Dis. 2019.

10. Dirks JA, Wolffs PF, Dukers-Muijrers NH, BrinkAA, Speksnijder AG, Hoebe CJ. Chlamydia trachomatis load in population-based screening and STI-clinics: implications for screening policy. PLOS One. 2015;10(3):e0121433.

11. van Liere GA, Dirks JA, Hoebe CJ, Wolffs PF, Dukers-Muijrers NH. Anorectal Chlamydia trachomatis Load Is Similar in Men Who Have Sex with Men and Women Reporting Anal Sex. PLoS One. 2015;10(8):e0134991.

12. Dirks JA, van Liere GA, Bogers S, Dukers-Muijrers NH, Wolffs PF, Hoebe CJ. Natural Course of Chlamydia trachomatis Bacterial Load in the Time Interval between Screening and Treatment in Anogenital Samples. PLoS One. 2015;10(12):e0145693.

13. Dukers-Muijrers $\mathrm{N}$, Wolffs PFG, Vries $\mathrm{H}$, et al. Treatment effectiveness of azithromycin and doxycycline in uncomplicated rectal and vaginal Chlamydia trachomatis infections in women: a multicentre observational study (FemCure). Clin Infect Dis. 2019. 

Section geographical analyses 


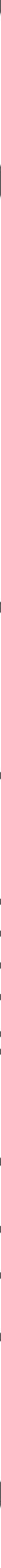





\section{Abstract}

\section{Background}

Standard epidemiological methods, such as regression models, are often used to identify high risk Chlamydia trachomatis (CT) and Neisseria gonorrhoeae (NG) geographical areas. Geographical cluster analyses can also be used for this purpose. Here, we compared the two approaches to assess the added value of geographical cluster analyses to inform regional STI clinic policy and to optimize control programs.

\section{Methods}

Coded national patient registry data from all 8 STI clinic regions in the Netherlands were analyzed. Data from all CT and NG test consultations of patients aged 15 up to 64 years between 2013 and 2015 were included ( $n=379,926)$. Two outcome measures were defined: the CT and NG detection rate. We performed two methods: (1) a multivariable Poisson regression analysis including geographical determinants and (2) a geographical cluster analysis using SaTScan including the 4-digit postal code of the patient. Additional number needed to test (NNT) to "capture" one CT or NG positive case was calculated for both methods per STI clinic region.

\section{Results}

The CT and NG detection rate varied per STI clinic region. Urban areas were associated with higher CT detection rates (OR:3.16;95\%Cl:3.09-3.23;P<0.001) and NG detection rates (OR:3.02;95\% Cl:2.89-3.16; $\mathrm{P}<0.001$ ). The geographical cluster analyses visualized clusters of high CT and NG detection rates in urban areas. In one region the geographical cluster analyses visualized no NG clusters, whereas $n=489$ NG cases were diagnosed. The NNT for CT and NG was lower for the geographical cluster analyses (range: 57-369) than the epidemiological analyses per STI clinic region (range: 93-2,104).

\section{Conclusion}

The added value of geographical cluster analyses lies in the detection and visualization of localized high-risk CT and NG areas and could be useful to efficiently target local STI control-outreach activities. A combination of both approaches is imperative in providing geographical insight in CT and NG testing and positivity. 


\section{Introduction}

Chlamydia trachomatis (CT) and Neisseria gonorrhoeae (NG) are the most diagnosed bacterial sexual transmitted infections (STIs), which both can be associated with complications in women such as pelvic inflammatory disease, infertility, and ectopic pregnancy. ${ }^{1}$ Centers for sexual health (CSH) serve key populations at high risk for CT and NG like young people aged below 25 years, men who have sex with men (MSM), and other groups with high risk for STIs. ${ }^{2}$ Despite control measures such as promoting safe sex as primary prevention, and active STI testing and treatment in risk groups, retesting and partner-management as secondary prevention, CT and NG positivity rates continue to rise. ${ }^{3}$

Individuals at risk for CT and NG share demographic and socioeconomic characteristics associated with CT and NG, and therefore may cluster geographically. ${ }^{45}$ The identification of these high-risk areas could inform local STI clinic policies to optimize local STI control by targeted interventions, such as outreach activities. ${ }^{67}$

Standard epidemiological methods, such as regression models, analyzing STI surveillance data could be used to identify high risk CT and NG areas. ${ }^{46-12}$ Standard epidemiological analyses include pre-defined geographical variables, such as states, counties and municipalities in large study areas to identify high risk areas. ${ }^{910} 12$ Recently, more-refined specialized geographical analyses and its use in public health have received increasing attention. ${ }^{5}$ In particular, geographical cluster detection software, such as SaTScan, is freely available and can be used to identify local STI clusters. ${ }^{13-16}$ Due to its ability to visualize high-risk STI areas, the results of SaTScan can serve as a basis for the prioritization of future interventions like enhanced testing and treatment. ${ }^{17}$ However, the application of this software requires knowledge of spatial epidemiological methods and Geographic Information Systems (GIS).

The question arises whether geographical cluster analyses have an added value to standard epidemiological analyses for public health STI control purposes. Both methods by their own capacity apply geographical information to inform local testing policies and outreach interventions. To date, no studies have compared an epidemiological approach with a geographical approach for the detection of high-risk CT and NG areas. Here, we assessed the added value of geographical cluster analyses compared to a standard epidemiological approach using our large scale national STI clinic database.

\section{Methods}

\section{Study population}

In this cross-sectional study, coded and anonymized CT and NG surveillance data from all 8 STI clinic regions in the Netherlands were obtained from the National Institute for Public 
Health and the Environment (RIVM). The geographical location of the 8 STI clinic regions can be found in earlier publications. ${ }^{314} 18$ Data from all CT and NG test consultations of patients aged 15-64 years with a known 4-digit postal code between 2013 and 2015 were included $(n=379,926)$ (Supplementary figure 1). Data were aggregated per 4-digit postal code to be able to compare the epidemiological analyses with the geographical cluster analyses.

\section{Main outcome measures}

We assessed two main outcome measures: (1) "CT detection rate" and (2) "NG detection rate", which are expressed as the proportion of CT or NG positive consultations per inhabitants aged 15-64 years per 4-digit postal code, respectively.

\section{Statistical analyses}

\section{Epidemiological analyses}

To assess geographical differences in CT- and NG detection rates per STI clinic region we performed multivariable Poisson regression analyses. For the Poisson regression analyses, we retrieved the number of 15-64 years old inhabitants per 4-digit postal code for the year 2014 from Statistics Netherlands (http://www.cbs.nl). The natural logarithm of the number of 1564 years old inhabitants per 4-digit postal code was set as the offset variable.

The variables indicative for geographical areas assessed were: coded STI clinic region ("A" to " $H$ "), urbanization and socioeconomic status. Urbanization was based on the definition of Statistics Netherlands (www.statline.nl); (very) highly urbanized ( $\geq 1,500$ (> 2500) addresses per squared kilometre) areas were categorized as "urban". Moderately, low urbanized areas ( $\geq 1,000-<1,500$ and $\geq 500-<1000$ addresses per squared kilometre) and rural ( $<500$ addresses per squared kilometre) areas were categorized as "non-urban". The SES score (SES; unknown, low, middle, high) was based on income, education level and employment extracted from the Netherlands Institute for Social Research per 4-digit postal code area (http://www.scp.nl).

The variable "STI clinic region" was included in the multivariable models, as this variable was of our main interest. Region "H" was used as the reference category, since the detection rate of region "H" was most close to the overall detection rate of the Netherlands. This enables comparison with the geographical analyses, since the denominator of the geographical analyses is the overall detection rate of the Netherlands. Factors with $p<0.10$ in the univariable Poisson regression analyses were included in the multivariable model. Relative risks (RR) and 95\% confidence intervals (95\% Cl) were calculated for the univariable and multivariable Poisson regression models. Only the multivariable results are presented. 
Analyses were performed using SPSS V21 (IBM SPSS Statistics for Windows, IBM Corporation, Armonk, New York, USA). A p value of $<0.05$ was considered statistically significant.

\section{Geographical cluster analyses}

We calculated the $\mathrm{CT}$ and $\mathrm{NG}$ detection rate with the relative risk (RR) per 4-digit postal code area. The RR estimates provide information how common CT and NG infections in a specific area are as compared to the global baseline (total number of infections divided by total number of inhabitants). The resulting raw CT and NG detection rates are unstable, as the number of inhabitants varies within the included 4-digit postal codes in the Netherlands. Therefore, we applied the conditional autoregressive Besag-York-Mollié (BYM) model without covariates to generate more stable CT and NG detection rates. ${ }^{19}$ The BYM model is a Poisson model where the number of observed cases is the dependent variable and the number of expected cases is the offset variable. The structured effect was assigned as the neighborhood structure of the municipalities, defined in this study as 4-digit postal codes sharing a common edge or border. ${ }^{20}$ The model was fitted using the integrated nested Laplace approximation (INLA), which is a computationally more practical alternative to Markov-Chain-Monte-Carlo simulations. ${ }^{21}$ The BYM model was fitted using the R package "R-INLA (www.r-inla.org)". The results were then imported in ESRI ArcGIS 10.2 for enhanced visualization.

The spatial scan statistic is a local cluster test, which identifies the location and the statistical significance of local clusters. ${ }^{22}$ We applied a Poisson purely spatial model where the number of CT and NG cases follow an inhomogeneous Poisson process. ${ }^{16}$ The input data for this model consisted of (1) the number of CT or NG positives per 4-digit postal code, (2) the number of the persons aged between 15 and 64 per 4-digit postal code, and (3) the centroids coordinates of each 4-digit postal code area. The statistical scan statistic imposes a circular scanning window, which is flexibly in size and position and gradually moves over all coordinates, evaluating all potential cluster locations and sizes up to either a user-defined maximum percentage of the population at risk or the default value of up to $50 \%$ of the population at risk. ${ }^{16}$ We set the maximum population at risk to not exceed $5 \%$ of the population. Previous research based on postal code level in the Netherlands found that a maximum cluster size of 5\% delivered practically useful results. ${ }^{17}$ The computation was carried out using SaTScan software version 9.4.1 (www. satscan.org) and were then imported in ESRI ArcGIS 10.2 for visualization.

\section{Number needed to test analyses}

To assess the potential added value of the geographical cluster analyses, we calculated the number needed to test (NNT) for the epidemiological analyses and the geographical cluster analyses. The NNT could provide valuable information for STI clinic policymakers for targeted testing strategies in certain geographical areas. The NNT was defined as the number of inhabitants who needed to be tested to "capture" one CT or NG positive patient per STI clinic 
region. The NNT was calculated by $1 /$ (detection rate) per STI clinic region for CT and NG separately. $^{23}$

The NNT for the epidemiological analyses was calculated based on a two-step approach. First, geographical areas (identified by urbanization degree and SES status) associated with higher CT/NG detection rates were identified using the earlier described Poisson regression analyses per STI clinic region. Second, additional CT and NG detection rates and NNT were calculated by only including CT or NG cases, and inhabitants living in these high-risk geographical areas within the STI clinic regions as this would be the target for outreach activities.

For the geographical cluster analyses, the CT and NG detection rates and NNT was calculated by only including cases and inhabitants living in the geographical clusters within the STI clinic regions. This enables a comparison between the epidemiological analyses and geographical cluster analyses as much as possible.

\section{Ethics statement}

Since the retrospective coded data originated from standard care and were analysed anonymously, neither a full ethical review nor informed consent for data analyses was needed, as confirmed and approved by the Medical Ethical Committee of Maastricht University (METC 2017-0251).

\section{Results}

\section{Epidemiological analyses}

CT detection rates, that is proportion of CT positive consultations per inhabitants aged 15-64 years per 4-digit postal code, varied between $0.26 \%$ and $0.84 \%$ in the STI clinic regions. NG detection rates varied between $0.03 \%$ and $0.23 \%$ in the STI clinic regions. Urban- and low SES areas had higher CT and NG detection rates in comparison with non-urban and high SES areas in multivariable Poisson regression analyses (Table 1). 


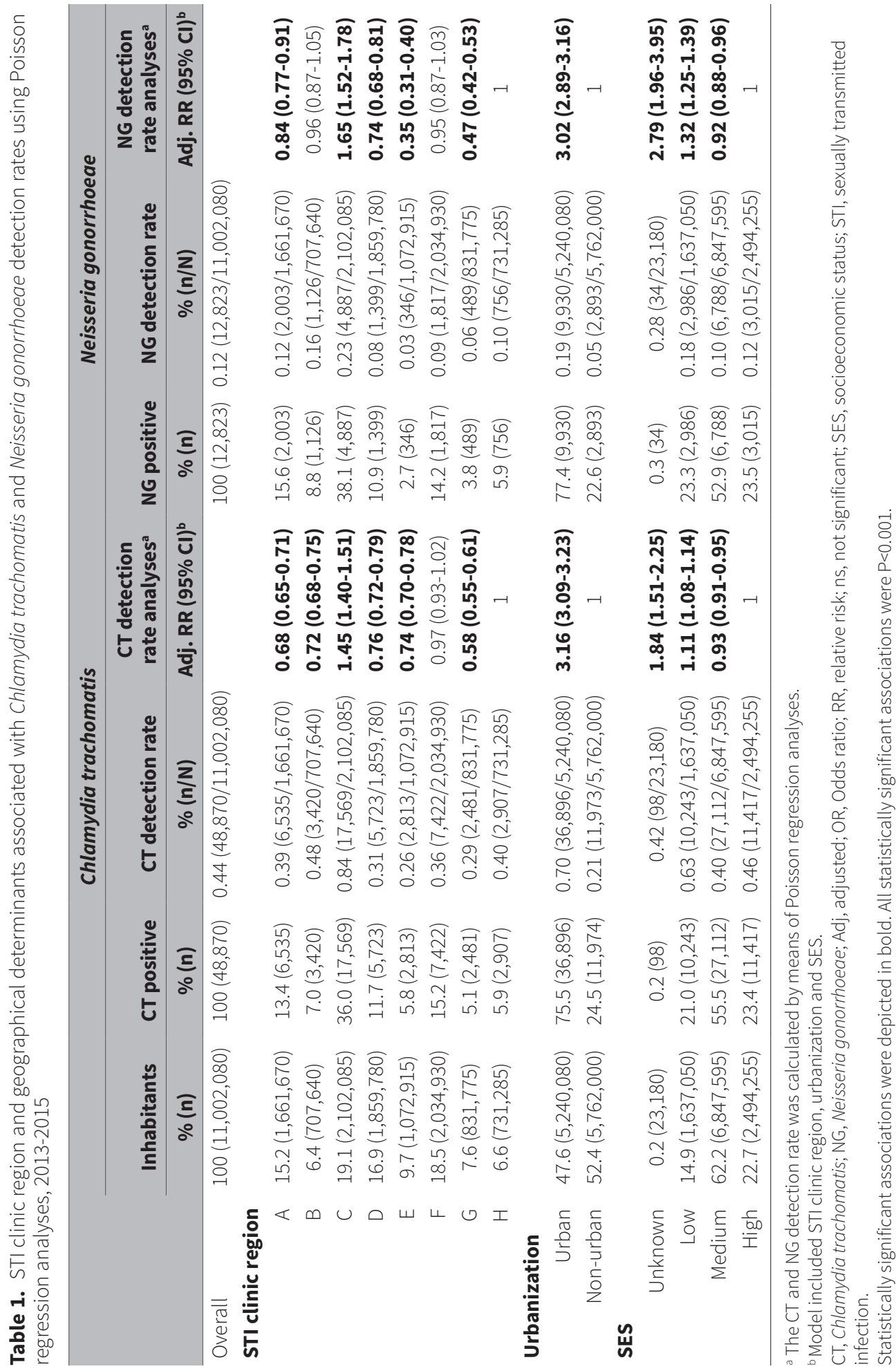




\section{Geographical cluster analyses}

The geographical cluster analyses detected 26 CT clusters which are visualized in Figure 1. Geographical clusters are 4-digit postal code areas with higher CT detection rates in comparison with the overall detection rate in the remaining 4-digit postal codes in the Netherlands.

The analyses detected $18 \mathrm{NG}$ clusters, and these are visualized in Figure 1. Both the CT and NG clusters were located in urbanized areas with high population densities.
A) Chlamydia detection rate
B) $\varnothing=116$ per 100,000
C) $\varnothing=478$ inhabitants per $\mathrm{km}^{2}$

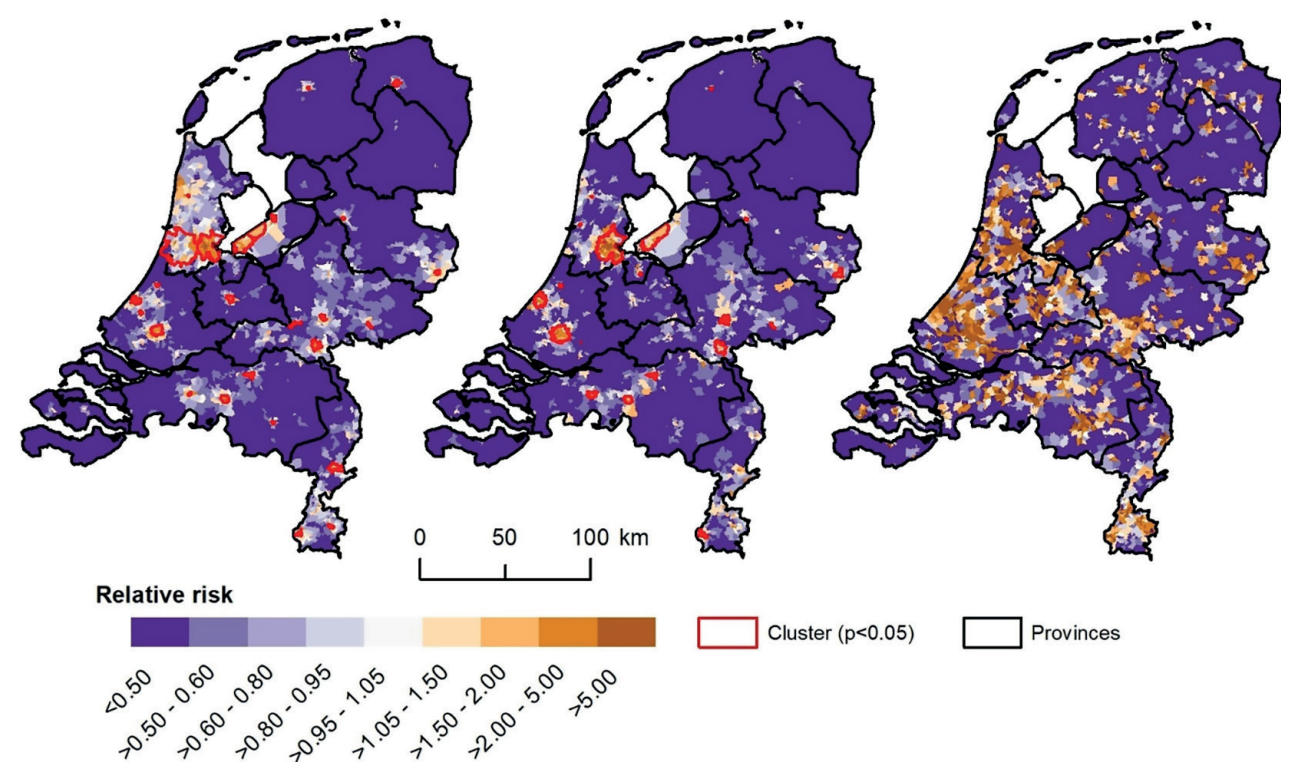

Figure 1. Results of the geographical cluster analyses for Chlamydia trachomatis and Neisseria gonorrhoeae, 2013-2015.

Maps including all provinces of the Netherlands. A) Visualization of the Chlamydia trachomatis detection rate. B) Visualization of the Neisseria gonorrhoeae detection rate. C) Visualization of the population density per squared kilometre. Statistically significant $(P<0.05)$ geographical clusters are depicted in red. 


\section{Number needed to test}

For the epidemiological NNT analyses, only patients living in urban or low SES areas were included per STI clinic region since these determinants were associated with higher CT and NG detection rates (Table 1). In general, the NNT for CT was higher for the epidemiological analyses (range 93-238) compared to the geographical analyses (range 57-122). This implies that more inhabitants are needed to be tested to capture one CT positive patient when applying the epidemiological analyses. The proportion of inhabitants living within the geographical clusters varied between 5.9\%-51.2\% per STI clinic region, whereas the proportion of captured CT positives within these geographical clusters varied between 24.1-80.3\% per STI clinic region (Table 2).

In seven out of eight STI clinic regions, the NNT for NG was higher for the epidemiological analyses (range 319-2,104) compared to the geographical cluster analyses (range 170-369). This means that more inhabitants are needed to be tested to capture one NG positive individual when using the epidemiological analyses. The proportion of inhabitants living within the geographical clusters varied between $0.4-34.0 \%$, whereas the proportion of captured NG positives varied between $7.2-77.8 \%$. The geographical cluster analyses visualized no geographical NG clusters in STI clinic region " $G$ ", whereas in total $n=489$ NG cases were diagnosed in this region (Table 3 ). 


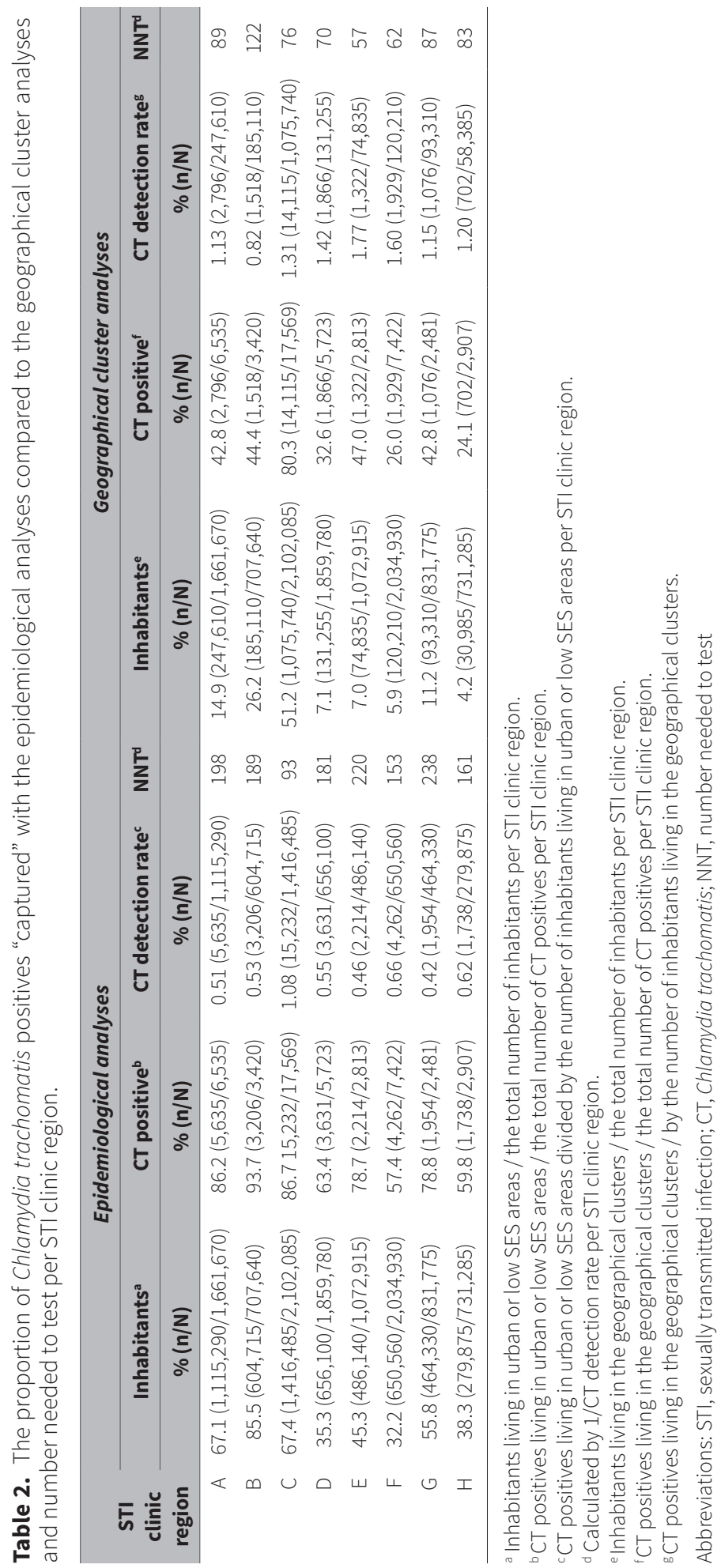




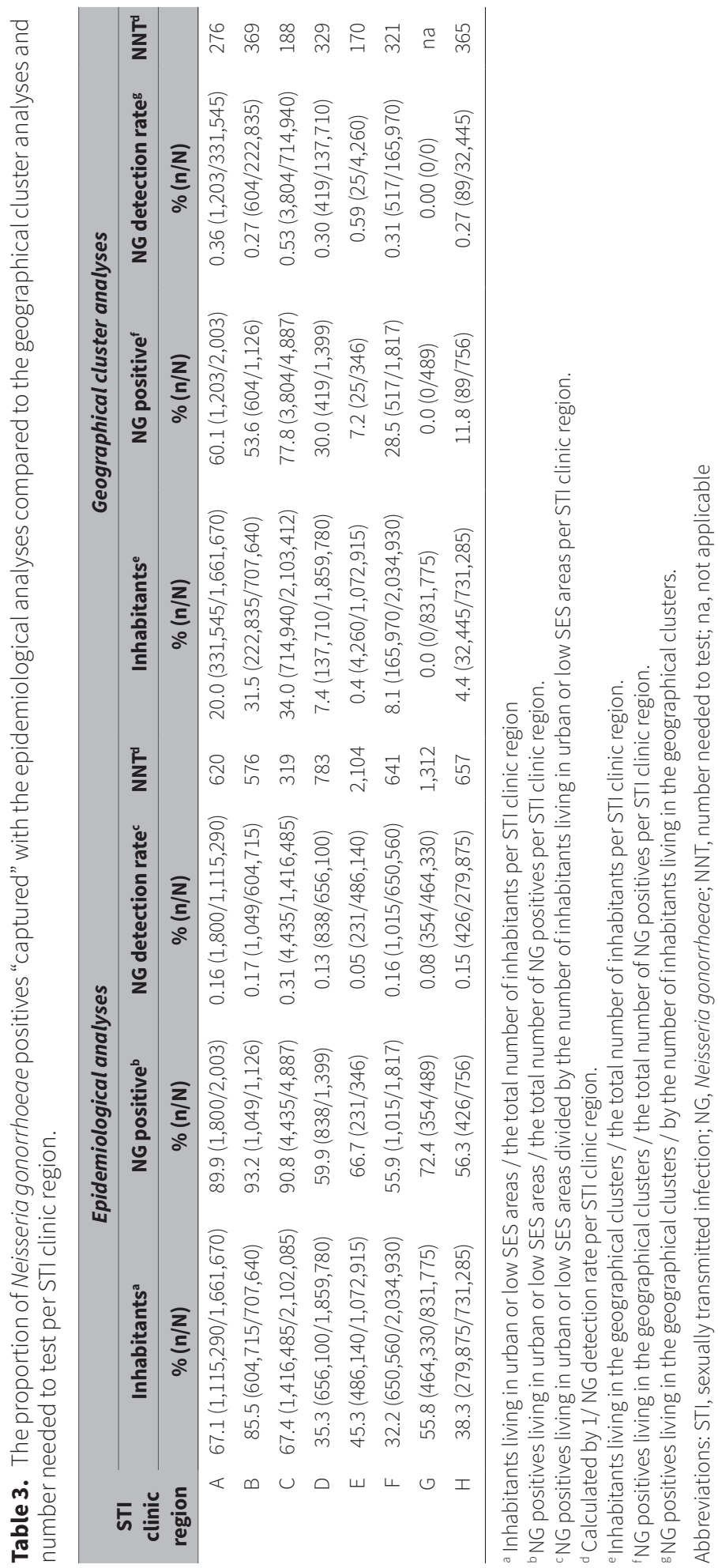




\section{Discussion}

In this study we analyzed large scale STI clinic surveillance data using two different methods, to assess the added value of geographical cluster analyses for public health STI control purposes. The epidemiological analyses showed higher CT and NG detection rates in urban areas and low SES areas. The geographical cluster analyses detected geographical CT and NG clusters in urban areas. The NNT for CT and NG was lower for the geographical cluster analyses compared to the epidemiological analyses in all STI clinic regions indicating the efficiency of geographical cluster analyses. However, in one STI clinic region the geographical cluster analyses did not visualize any NG cluster, whereas n=489 NG cases were diagnosed in this region. Therefore, the combination of both methods is needed to obtain insight in a whole country and to avoid missed cases.

We quantified the number of CT and NG positives captured with the geographical cluster analyses, calculated the NNT, and assessed variations per STI clinic region. The cluster analyses provide visual results of the analyses, which are attractive to read. However, the actual added value of geographical cluster analyses for targeted STI testing seems dependent of the size of the study area of interest and the purpose of the testing strategy. This means that in some areas a substantial proportion of the inhabitants are needed to be tested to capture a large proportion of CT positives, whereas in other areas a lower proportion of inhabitants are needed to be tested to capture a large part of the CT positives. The number of captured CT positives was always lower for the geographical cluster analyses compared to the epidemiological analyses. Given the small resolution of the Dutch 4-digit postal codes (approximately four thousand), however, this is in part due to the computation of significance ranks for each possible cluster using Monte-Carlo replications. This means that SaTScan would detect those clusters ranked as significant by the Monte-Carlo replications when the analysis is run for only a subset of the region, such as provinces, which would have been ranked as insignificant when the analysis is performed for the whole of the Netherlands. To detect more cases, the cluster analysis should be performed for subsets of the region (e.g. provinces). Despite this limitation, the number of inhabitants living in the geographical clusters was much lower than the inhabitants needed to be tested with the epidemiological analyses. Therefore, testing based on geographical cluster analyses could be preferred cost wise, as cluster-based testing requires a much smaller number of inhabitants to be tested to capture a positive case. However, it would also fail to capture a substantial part of cases when run for a whole country at such fine spatial resolution. Therefore, STI control professionals should decide on the purpose of their testing strategies: (1) capture as much as possible CT/NG infections by testing a large part of the population within an STI clinic region (epidemiological analyses), or (2) capture approximately half of the CT/NG infections by only testing a small proportion of the inhabitants of the STI clinic region mostly living in urban areas within the geographical clusters (geographical cluster analyses). 
The geographical cluster analyses did not visualize any NG clusters in STI clinic region " $G$ ". It is possible that NG cases are more dispersed in this area or that a possible cluster was not ranked as significant within the cluster analysis for the whole of the Netherlands. Thus, defining policy only based on NNT and geographical cluster analyses alone without the epidemiological analyses could lead to missed cases in areas without geographical clustering of cases or a repeated cluster analysis of a subset of the region.

A strength of the current study was the inclusion of all CT and NG tests performed by all STI clinics in the Netherlands between 2013 and 2015. This is the first study comparing an epidemiological approach to a geographical cluster approach to detect high risk CT and NG areas in a whole country.

Nevertheless, the current study had several limitations. First, only STI clinic data was analyzed, while about half to two thirds of all CT and NG infections in the Netherlands are diagnosed by general practitioners (GP). ${ }^{324}$ Maps including GP data could lead to different clusters. We assume that patients in non-urban areas visit the GP more often than STI clinics, as most STI clinics are located in urban areas. Additional research is needed to assess this pattern. Also, a previous Dutch study observed much higher CT prevalence rates in highly urbanized areas compared to rural areas. ${ }^{25}$ Second, we could not adjust for repeat testing of patients. As STI clinics serve risk groups such as youngsters and MSM, it is likely that patients have repeat visits within the three-year study period. Also, patients who test positive for CT and/or NG are advised to retest within one year as repeat infections frequently occur. ${ }^{26}$ Therefore, the CT or NG detection rate per 4-digit postal code could represent multiple positive tests by a single individual leading to some overestimation. Furthermore, we included the 4-digit postal code of the patients' residence. However, this location was not necessarily where sexual activity had taken place. ${ }^{15}$ This could lead to further transmission of CT and NG in other locations than the detected clusters. ${ }^{15}$ The variable "STI clinic region" was based on the 4-digit postal code of the patients' region of residence. Therefore, we were unable to assess whether the consultation was indeed held at the STI clinic in the STI clinic region where the patient lives. However, we expect patients travel only a few kilometers to an STI clinic as suggested by a study in Hongkong. ${ }^{27}$ A general disadvantage of geographical cluster analyses is that the use of different sizes of geographical units, such as 4-digit postal codes or municipalities, could lead to different clusters and therefore different results. ${ }^{17}$ This is also known as modifiable areal unit problem (MAUP). ${ }^{17}$ Known demographic risk factors for CT and NG, such as sex and age, were not assessed in the current study. This was beyond our scope of interest as we assessed the added value of cluster analyses including only geographical determinants.

In conclusion, geographical cluster analyses have added value in visualizing data, detecting high risk CT and NG clusters, and when used as STI testing strategy for efficient testing in high risk areas. This all could be useful for decision makers to guide STI clinic policy and optimize CT and NG control efforts. However, interpretation of geographical cluster analyses for a whole country is difficult as geographical clusters included the minority of CT and NG infections, and insight in the total picture is imperative to make policy decisions. Additional 
geographical cluster analyses on a smaller scale (e.g. province level) would provide additional geographical information. Moreover, an epidemiological analysis is always needed since geographical areas without geographical clusters might have more dispersed cases. Altogether, both approaches provide different and valuable information. Therefore, a combination of both approaches is necessary for providing geographical insight in CT and NG testing and positivity. 


\section{References}

1. Haggerty CL, Gottlieb SL, Taylor BD, et al. Risk of sequelae after Chlamydia trachomatis genital infection in women. J Infect Dis 2010;201 Suppl 2:S134-55. doi: 10.1086/652395.

2. den Heijer CD, van Liere GA, Hoebe CJ, et al. Who tests whom? A comprehensive overview of Chlamydia trachomatis test practices in a Dutch region among different STI care providers for urogenital, anorectal and oropharyngeal sites in young people: a cross-sectional study. Sex Transm Infect 2016;92(3):211-7. doi: 10.1136/sextrans-2015-052065.

3. Slurink I, van Aar F, Op de Coul E, et al. Sexually transmitted infections in the Netherlands in 2018: Rijksinstituut voor Volksgezondheid en Milieu.

4. Ellen JM, Hessol NA, Kohn RP, et al. An investigation of geographic clustering of repeat cases of gonorrhea and chlamydial infection in San Francisco, 1989-1993: evidence for core groups. J Infect Dis 1997;175(6):1519-22.

5. Jennings JM, Curriero FC, Celentano D, et al. Geographic identification of high gonorrhea transmission areas in Baltimore, Maryland. Am J Epidemiol 2005;161(1):73-80. doi: 10.1093/aje/ kwi012.

6. Gale M, Hayen A, Truman G, et al. Demographic and geographical risk factors for gonorrhoea and chlamydia in greater Western Sydney, 2003-2013. Commun Dis Intell Q Rep 2017;41(2):E134-E41.

7. Lacey CJ, Merrick DW, Bensley DC, et al. Analysis of the sociodemography of gonorrhoea in Leeds, 1989-93. BMJ 1997;314(7096):1715-8.

8. Miranda AE, Silveira MF, Travassos AG, et al. Prevalence of Chlamydia trachomatis and Neisseria gonorrhea and associated factors among women living with Human Immunodeficiency Virus in Brazil: a multicenter study. Braz J Infect Dis 2017;21(4):402-07. doi: 10.1016/j.bjid.2017.03.014.

9. Ginocchio CC, Chapin K, Smith JS, et al. Prevalence of Trichomonas vaginalis and coinfection with Chlamydia trachomatis and Neisseria gonorrhoeae in the United States as determined by the Aptima Trichomonas vaginalis nucleic acid amplification assay. J Clin Microbio/ 2012;50(8):2601-8. doi: 10.1128/JCM.00748-12.

10. Miller WC, Ford CA, Morris M, et al. Prevalence of chlamydial and gonococcal infections among young adults in the United States. JAMA 2004;291(18):2229-36. doi: 10.1001/jama.291.18.2229.

11. McDonnell DD, Levy V, Morton TJ. Risk factors for Chlamydia among young women in a northern california juvenile detention facility: implications for community intervention. Sex Transm Dis 2009;36(2 Suppl):S29-33. doi: 10.1097/OLQ.0b013e31815dd07d.

12. James AB, Geisler WM. Predictors of high chlamydia and gonorrhea positivity rates among men in the southern United States. J Natl Med Assoc 2012;104(1-2):20-7.

13. van Aar F, den Daas C, van der Sande MAB, et al. Outbreaks of syphilis among men who have sex with men attending STI clinics between 2007 and 2015 in the Netherlands: a space-time clustering study. Sexually Transmitted Infections 2017;93(6):390-95. doi: 10.1136/sextrans-2016-052754.

14. Soetens LC, van Benthem BH, Urbanus A, et al. Ongoing transmission of hepatitis B virus in rural parts of the Netherlands, 2009-2013. PLoS One 2015;10(2):e0117703. doi: 10.1371/journal. pone.0117703. 
15. Bush KR, Henderson EA, Dunn J, et al. Mapping the core: chlamydia and gonorrhea infections in Calgary, Alberta. Sex Transm Dis 2008;35(3):291-7. doi: 10.1097/OLQ.0b013e31815c1edb.

16. Kulldorff M. A spatial scan statistic. Communications in Statistics-Theory and methods 1997;26(6):1481-96.

17. Kauhl B, Heil J, Hoebe CJ, et al. The Spatial Distribution of Hepatitis C Virus Infections and Associated Determinants--An Application of a Geographically Weighted Poisson Regression for Evidence-Based Screening Interventions in Hotspots. PLoS One 2015;10(9):e0135656. doi: 10.1371/ journal.pone.0135656.

18. Gotz HM, van Oeffelen LA, Hoebe C, et al. Regional differences in chlamydia and gonorrhoeae positivity rate among heterosexual STI clinic visitors in the Netherlands: contribution of client and regional characteristics as assessed by cross-sectional surveillance data. BMJ Open 2019;9(1):e022793. doi: 10.1136/bmjopen-2018-022793.

19. Besag J, York J, Mollié A. Bayesian image restoration, with two applications in spatial statistics. Annals of the Institute of Statistical Mathematics 1991;43(1):1-20. doi: 10.1007/BF00116466.

20. Roger S. Bivand EP VG-R. Applied spatial data analysis with R. New York 2013.

21. Rue H, Martino S, Chopin N. Approximate Bayesian inference for latent Gaussian models by using integrated nested Laplace approximations. Journal of the Royal Statistical Society: Series B (Statistical Methodology) 2009;71(2):319-92. doi: 10.1111/j.1467-9868.2008.00700.x.

22. Tanser F, Barnighausen T, Cooke GS, et al. Localized spatial clustering of HIV infections in a widely disseminated rural South African epidemic. Int J Epidemiol 2009;38(4):1008-16. doi: 10.1093/ije/ dyp148.

23. Rembold CM. Number needed to screen: development of a statistic for disease screening. BMJ 1998;317(7154):307-12

24. van den Broek IV, Verheij RA, van Dijk CE, et al. Trends in sexually transmitted infections in the Netherlands, combining surveillance data from general practices and sexually transmitted infection centers. BMC Fam Pract 2010;11:39. doi: 10.1186/1471-2296-11-39.

25. van Bergen J, Gotz HM, Richardus JH, et al. Prevalence of urogenital Chlamydia trachomatis increases significantly with level of urbanisation and suggests targeted screening approaches: results from the first national population based study in the Netherlands. Sex Transm Infect 2005;81(1):17-23. doi: 10.1136/sti.2004.010173.

26. Hosenfeld CB, Workowski KA, Berman S, et al. Repeat infection with Chlamydia and gonorrhea among females: a systematic review of the literature. Sex Transm Dis 2009;36(8):478-89. doi: 10.1097/OLQ.0b013e3181a2a933.

27. Lee SS, Ho KM, Cheung GM. The spatial context of clinic-reported sexually transmitted infection in Hong Kong. BMC Infect Dis 2010;10:275. doi: 10.1186/1471-2334-10-275. 



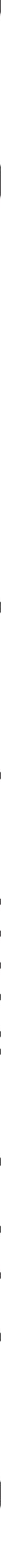




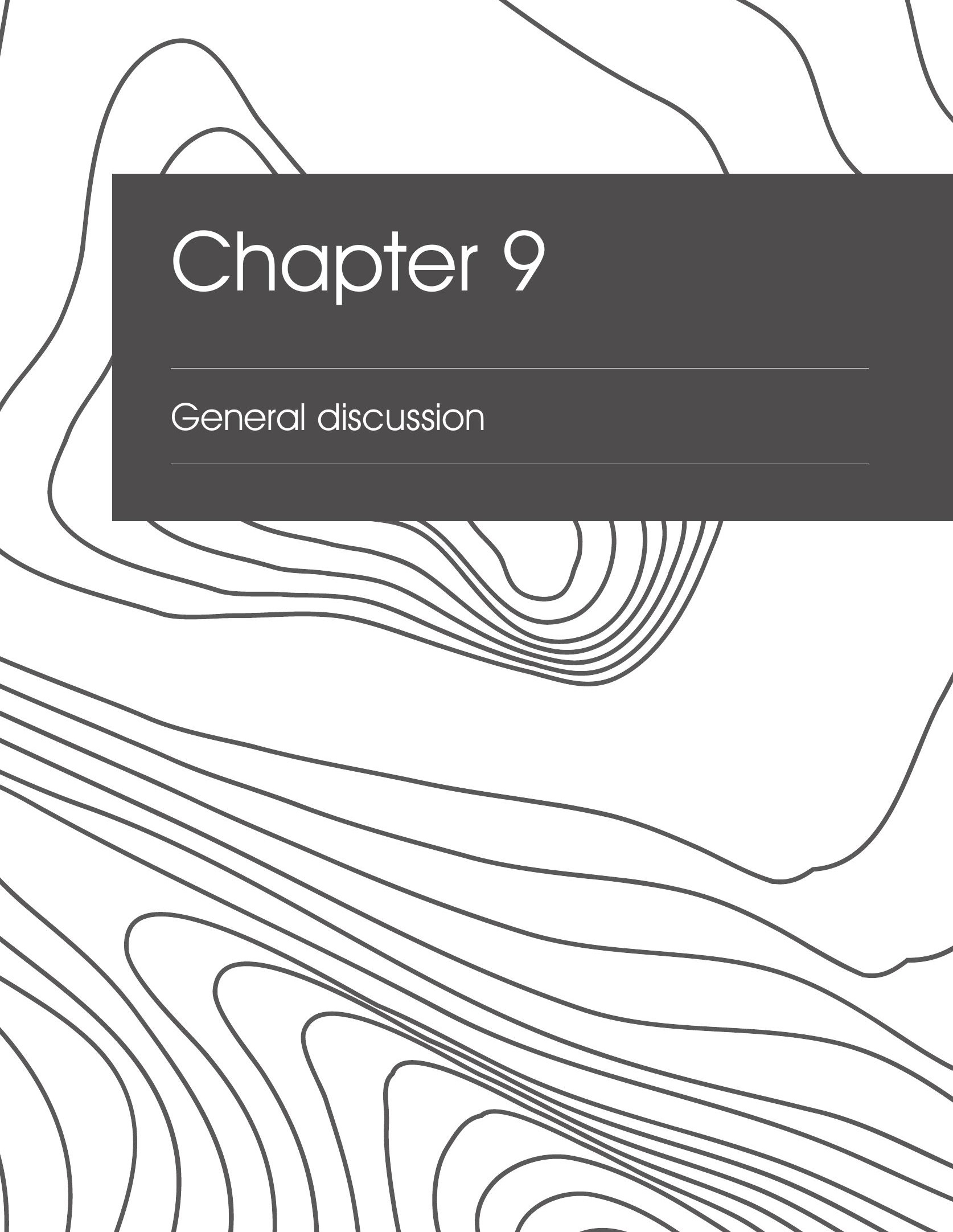


The studies in this thesis include epidemiological studies and geographical mapping to enhance and inform Chlamydia trachomatis (CT) and Neisseria gonorrhoeae (NG) control. The studies provide new insights in test practices of general practitioners (GPs), who has repeat CT and NG infections, the CT bacterial load at different anatomic sites, and the value of geographical mapping of CT and NG for control, in addition to conventional epidemiological methods. The main findings of this thesis are discussed in this chapter. Also, recommendations for future research and CT and NG control policy are provided.

\section{Test of cure, retesting and extragenital testing}

GPs are key providers of sexually transmitted infections (STI) care in the Netherlands as they perform a majority of tests and diagnose large part of CT and NG infections. ${ }^{12}$ In chapter 2 of this thesis we describe a study on the use of a test of cure (TOC) in general practice. A TOC is a CT or NG diagnostic test after first diagnosis and treatment to see if the infection has resolved. We showed that in the GP setting, the test of cure (TOC) rate of CT and NG patients is high (CT: 20\%, NG: 25\%). This is especially high considering the fact that the recommendation is not to perform a TOC after positive CT or NG test according to the Dutch national GP guidelines, and only perform a TOC in specifically indicated cases, such as (1) patients treated with an alternative regimen, (2) pregnant patients, (3) patients with persistent or recurrent symptoms or (4) patients re-exposed to an untreated partner. ${ }^{3}$ Notably, except for pregnant treated patients (TOC after 4-6 weeks), no time frame for TOC is given. It is likely that the majority of TOCs were not indicated in the GP guideline. Performing TOC might lead to false-positive results due to non-viable CT or NG that still may be present in the first week after treatment. ${ }^{4}$ Moreover, this might lead to overtreatment. The British Association for Sexual Health and HIV (BASHH) and the European guideline on the diagnosis and treatment of NG in adults recommend a TOC for all patients infected with NG 2 weeks after treatment due to increasing multidrug resistance of NG. ${ }^{5}$ Whereas the CDC and the World Health Organization (WHO) only advice a NG TOC in specifically indicated cases. ${ }^{78}$ Besides that, the exact timing of a TOC for NG remains a matter of debate. ${ }^{4}$ To date, no NG cases with antimicrobial resistance to first line treatment ceftriaxone are diagnosed in the Netherlands yet. ${ }^{1}$ Therefore, a routine TOC for all NG cases is not needed. ${ }^{9}$ However, focus should be on those specifically indicated cases with remaining symptoms, patients treated with an alternative regimen, pregnant patients and those re-exposed to an untreated partner.

Another study topic in the study was the number of retests performed by GPs. Repeat test or retest are done in CT or NG positive patients as studies have shown that positive tests are frequently followed by reinfection of the same patient. For $\mathrm{CT}$, reinfection rates are reported up to $32 \%$ and for NG up to $40 \% .^{10}{ }^{11}$ Despite the fact that retesting CT and NG positive patients within 3 to 12 months is advised in many international guidelines, we observed low retesting rates among GP patients. . $^{5-72}$ The retesting rate (between 3-12 months) in patients 
with CT is $24 \%$ and with NG is $15 \%$, which we consider relatively low. Retesting CT and NG positive patients was not advised in the Dutch national guideline for GPs (2005). In the latest Dutch national guideline for GPs (2013), GPs are recommended to advice CT positive patients to retest within one year. ${ }^{3}$ Remarkably, no retesting recommendations for NG are given despite relatively high reinfection rates within one year of a previous infection. ${ }^{1011}$ In our study (chapter 2), 12\% tested CT positive within 3 to 12 months. Due to the fact that only one out of four patients were retested, reinfections were likely missed, potentially leading to ongoing transmission of CT. No reinfections for NG were observed, probably due to the low number of retesting patients ( $n=11$ ). To facilitate retesting at the GP practice, GPs could consider using modern testing and communication strategies such as e-health. For example, GPs could, in collaboration with their diagnostic laboratory and public health services, send home sampling kits 3 to 12 months post treatment to improve retesting rates. Also, automatic small text messages could be used. These two methods have shown to increase retesting rates in STI clinic settings. ${ }^{13} 14$ Moreover, education and awareness of the importance of CT and NG testing could improve (re)testing rates at the GP practice. ${ }^{15}$ Recently, a study in New Zealand showed that a combination of clinician education, advice, and SMS text reminders increased $\mathrm{CT}$ and $\mathrm{NG}$ retesting rates significantly in primary care. ${ }^{16}$

In this study we also assessed extragenital testing, meaning diagnostic testing of the anorectal and oropharyngeal anatomical site. For specific risk groups like men who have sex with men this is warranted. A notable finding was that in only $0.3 \%$ of the consultations GP patients were tested at extragenital sites (chapter 2). An earlier study (data until the year 2010) by colleagues den Heijer et al. showed also very low $(<1 \%)$ extragenital testing rates of CT and NG among GPs in a comparable region in Limburg. ${ }^{917}$ Thus, the extragenital testing rates by GPs have not increased until the year 2016 despite that these data were communicated to GPs in the region at several occasions (expert-meetings) and despite many national (e.g. in the national GP journal 'Huisarts \& Wetenschap') and international scientific publications showing that extragenital infections are common among women and MSM. ${ }^{18}$ Moreover, it is estimated that $9 \%$ of the men visiting the GP are MSM, in which anorectal testing should be performed. ${ }^{319}$ As MSM are disproportionally affected by NG testing, retesting and treating this high risk group remains important to enhance NG control.

\section{Repeat Chlamydia trachomatis and Neisseria gonorrhoeae infections}

In chapter $\mathbf{3}$ and $\mathbf{4}$ of this thesis, we assessed whether patient repeatedly infected with CT or NG had different sociodemographic characteristics compared to patients frequently tested negative for CT and NG. Indeed, profiles in patients with repeat CT and NG infections reflected the high risk groups as described in testing guidelines, such as MSM and individuals younger than 25 year of age..$^{20}$ However, there was a notable difference in the sex-distribution in patients with $\mathrm{CT}$, and patients with NG repeat infections. 
According to our analyses, there was no significant difference of repeat CT infections between men who have sex with women (MSW) (19\%) and MSM (20\%), and although higher, this was also not significantly different for women (61\%) ( $P=0.32$ ). In contrast, the distribution of NG repeat infections was unequally distributed and was mainly focused in MSM: 7\% MSW, 83\% MSM, and 10\% women ( $p<0.001$ ). Others report that patients repeatedly infected with STIs are part of, so called, "core-groups" who have disproportionally impact on transmitting and acquiring STIs. ${ }^{21}$ We believe that this is likely true for NG, but not for CT. The vast majority of NG infections (as well as repeat infections) in the Netherlands are diagnosed among MSM (76\%). ${ }^{1}$ This indicates that NG is an STI mainly affecting MSM. CT is more widespread affecting the general population, including youngsters, men, and women.

\section{Chlamydia trachomatis bacterial load}

As viral load, the number of virus particles in a given volume of body fluid, is in some STIS associated with increased risk for transmission (such as in HIV and herpes simplex virus). This might be likewise true for the CT bacterial load. The CT bacterial load is frequently expressed as the number of bacteria/milliliter. The $\mathrm{CT}$ bacterial load can be measured directly or indirectly by using the cycle quantification value (Cq value). The $\mathrm{Cq}$ value of a real time nucleic acid quantification test (NAAT) is a measure of the number of cycles needed to give a positive signal in the diagnostic test and is thus an approximation of the inverse CT bacterial load. The CT bacterial load may hypothetically affect transmission potential and possibly also the development of sequelae. In chapter $\mathbf{5}$ of this thesis, we assessed whether the urogenital CT bacterial load, based on Cq values, was different between populations visiting STI clinics, GPS, and hospital physicians. We hypothesized that STI clinic patients have a higher urogenital CT load compared to GP and hospital physician patients, since STI clinics specifically target high risk individuals. However, we observed that in men no differences were observed between STI care-provider populations. Whereas, women visiting GPs had lower urogenital Cq values (higher CT load) compared to women visiting the STI clinic (30.2 vs. 30.9; $p=<0.001$ ). This finding was in contrast with our hypothesis. Although, the small difference observed $(0.7 \mathrm{Cq})$ is worth discussing. Translating this number into a "factor CT load", which means the number of times the CT bacterial load was higher between the studied groups, this would be $2^{\wedge} 0.7 \mathrm{Cq}=1.6$. Thus, women visiting the GP have a 1.6 times higher urogenital $\mathrm{CT}$ load compared to women visiting the STI clinic. The clinical and microbiological relevance of the observed difference remains unclear. Earlier, our study group deemed a factor CT load of 10 $(3.3 \mathrm{Cq})$ as clinically relevant to overcome potential technical variations when measuring the CT load within the same patient over time. ${ }^{22}$ In chapter $\mathbf{5}$ and $\mathbf{6}$ of this thesis we averaged the CT load over an entire population. For example, in chapter $\mathbf{5}$, the GP, STI clinic, and hospital population and in chapter $\mathbf{6}$ all patients with, urogenital, anorectal or oropharyngeal CT. Therefore, potential technical differences between individuals are flattened out and likely 
have little impact on the results, because these potential variations are randomly distributed over all samples tested within the particular group. Nevertheless, the precise relevance of the differences in CT load between groups remains unknown.

A notable finding of chapter $\mathbf{5}$ was that women concurrently infected with urogenital and anorectal CT had an 8.5 times higher urogenital CT load compared to women with only a urogenital CT infection. Moreover, women who were not anorectally tested also had an 8.1 times higher urogenital CT load compared to women with a urogenital CT infection. This could indicate that women who were not anorectally tested also had an anorectal CT infection, since anorectal CT infections among women are highly common. ${ }^{18}$ As a consequence, women with a urogenital CT infection and an untested anorectal CT infection are likely treated with azithromycin, since azithromycin is the first choice treatment for urogenital CT in the Netherlands. ${ }^{20}$ Therefore, potential undiagnosed anorectal $\mathrm{CT}$ infections may not be adequately treated as the efficacy of doxycycline exceeds that of azithromycin in anorectally positive women. ${ }^{23}$ Those women with untested anorectal CT infections could have impact on transmission by auto-inoculation, and possibly the progression to sequelae, as we are currently studying in FemCure. ${ }^{2324}$

An earlier publication by Dirks et al., including 28 CT load based studies, showed that the CT load was measured and expressed in 14 different ways in the literature. ${ }^{25}$ This hampers comparison between studies and standardization is needed. ${ }^{25}$ In chapter $\mathbf{6}$, we assessed whether the CT load of urine samples, vaginal swabs, oropharyngeal swabs and anorectal swabs were different in men and women. To date, this is the largest study ( $n=7,900$ CT positive samples) assessing the CT load of different anatomic locations in one study using the same molecular analysis method and the same protocol per anatomical locations for all patients sampled. We showed that vaginal swabs had the highest CT load followed by urine samples, anorectal swabs and oropharyngeal swabs. Oropharyngeal CT infections with low CT loads tend to clear more often than those with high CT loads which may indicate that samples with low loads have less impact on transmission. ${ }^{26}$ Nevertheless, oropharyngeal samples with a high CT load could act as a reservoir for CT as a study showed that one out of three women, and more than half of MSM have an oropharyngeal CT infection without a concurrent anogenital CT infection. ${ }^{26} \mathrm{Nev}$ ertheless, the median clearance time for oropharyngeal CT is still unclear.

The most notable finding in chapter $\mathbf{6}$ was that men and women have an equal oropharyngeal CT load and an equal anorectal CT load. This finding could fuel discussion about whether all women, just as is now standard practice in MSM, need to be tested anorectally. Moreover, relevance in women might be even more substantial as they bear the burden of reproductive morbidity caused by $\mathrm{CT}^{2}{ }^{27}$

Furthermore, we showed that CT patient co-infected with other STIs such as NG and HIV have a lower urogenital CT load (chapter 6). In an additional analysis, we showed that those patients (high risk group) were more often repeatedly infected with CT potentially leading to some partial immunity against CT. In chapter $\mathbf{7}$ we confirmed this finding; women and men repeatedly infected with $\mathrm{CT}$ have a lower urogenital CT load. The partial immunity theory is 
endorsed by other studies. ${ }^{2829}$ Although, the precise mechanism and interaction with different treatments (azithromycin and doxycycline) remains unknown. Likely treatment with azithromycin or doxycycline could play a role in the extent to which partial immunity exist for CT. As treatment with doxycycline exceeds that of azithromycin in urogenital and anorectal CT ${ }^{23} 3031$, partial immunity against CT could be higher in patients treated with azithromycin. However, this is only speculative and further research is needed to confirm or reject this hypothesis.

\section{Epidemiological versus geographical analyses}

In chapter $\mathbf{8}$ of this thesis we assessed the added value of geographical cluster analyses compared to epidemiological analyses for detecting high risk CT and NG areas using Dutch national surveillance data. The geographical analyses showed various high-risk geographical clusters mainly in areas with high populations densities. The epidemiological analyses showed also high CT and NG detection rates in urban areas.

In contrast, in one STI clinic region ("G") the geographical cluster analyses visualized no NG clusters indicating no high-risk area. However, according to the epidemiological analyses, 354 NG cases were identified when including low SES and urban areas within that specific STI clinic region. Therefore, assessing the geographical cluster analyses without the epidemiological analyses would lead to missed cases. Furthermore, the number of "captured" CT positives was always lower for the geographical cluster analyses compared to the epidemiological analyses. An additional geographical cluster analyses of a subset of the region (for example provinces), would lead to different geographical clusters with detailed information which can be used for potential targeted outreach activities. This highlights the difficulty of interpreting geographical mapping data which always should be done with caution and in combination with other epidemiological data.

The NNT for the geographical cluster analyses was in all STI clinic regions (expect for region "G") much lower than the epidemiological analyses. Thus, despite that the geographical cluster analyses included a minority of CT and NG cases, it can be used to efficiently target resources for outreach activities. Therefore, STI control professionals should decide on the purpose of their testing strategies: (1) capture as much as possible CT/NG infections by testing a large part of the population within an STI clinic region (epidemiological analyses), or (2) capture approximately half of the CT/NG infections by only testing a small proportion of the inhabitants of the STI clinic region mostly living in urban areas within the geographical clusters (geographical cluster analyses). We believe that a combination of both methods is needed to provide insight in an entire region and to avoid potential missed cases. Furthermore, the application of geographical analyses software requires expert knowledge on its use and the interpretation of the results. A general disadvantage of geographical cluster analyses is that the use of different sizes of geographical units, such as 4-digit postal codes or munici- 
palities, could lead to different clusters and therefore different results. This is also known as modifiable areal unit problem (MAUP).

To conclude, all patients diagnosed with CT or NG should be retested within 3 to 12 months after diagnosis. Especially in the GP setting, we observed relatively low CT and NG retesting rates. The currently ongoing "Chlamydia: Hidden Infectious Management in Primary care" (CHIMP) study could resolve some knowledge gaps in CT and NG control in the GP setting. First, it will answer the question whether retesting rates will increase by sending an email or SMS six months after diagnosis to request a home sampling kit for self-testing. Second, it will answer the urgent question whether anorectal CT infections are common among urogenital CT positive women visiting the GP. The latter would have impact on testing and treatment guidelines for GPs. If it turns out that the majority of urogenital CT positive women visiting the GP also have an anorectal CT infection. Then potentially all women with a urogenital CT infection should routinely be treated with doxycycline instead of azithromycin. ${ }^{23}$ Routine anorectal testing will lead to extra healthcare costs. When all urogenital CT positive women are treated with doxycycline, then extra anorectal testing is not needed. However, doxycycline treatment adherence could be an issue, since doxycycline should be taken twice a day for seven days. This could possibly lead to not fully treated CT infections. Practical care strategies, such as automatic text messages or online applications, could potentially help treatment adherence. ${ }^{23}$

The most important question that remains unanswered is whether a high bacterial CT load increases transmission. I believe it may, but further complex research is needed to assess this pattern. Likely patients with a low CT bacterial load may not transmit CT, like the viral load of HIV (not detectable = not transmittable). Hypothetically, in the future, patients with a low CT bacterial load may not be treated leading to less overtreatment, potential less antimicrobial resistance and less healthcare costs. Furthermore, conventional highly sensitive qPCR techniques cannot distinguish between viable and non-viable CT DNA. Therefore, potential non-viable CT infections may be treated leading to overtreatment. Recently, our study group invented a viability PCR (V-PCR) technique in which viable and non-viable CT DNA can be distinguished. ${ }^{32}$ Moreover, they showed that in approximately half of the anorectal CT positive women viable CT DNA could be detected. ${ }^{33}$ Hypothetically, V-PCR could play are role in the future for whether or not treating CT positive patients. The currently ongoing "CHLAMOUR" study of the public health service South Limburg will provide insight in (1) the viability of CT at different anatomic locations, (2) the most important anatomical location for CT transmission, and (3) insight in enhanced CT treatment.

The potential added value of geographical cluster analyses could be tested in practice. For example, an additional cluster analyses per province or STI clinic region would lead to more detailed information and additional geographical clusters on a smaller scale. Based on these results, outreach testing activities could take place in these specific areas to detect potential hidden CT and NG infections. The results would reveal the practical added value of geographical cluster analyses. 


\section{References}

1. Slurink I, van Aar F, Op de Coul E, et al. Sexually transmitted infections in the Netherlands in 2018: Rijksinstituut voor Volksgezondheid en Milieu.

2. Slurink I, Groen K, Gotz HM, et al. Contribution of general practitioners and sexual health centres to sexually transmitted infection consultations in five Dutch regions using laboratory data of Chlamydia trachomatis testing. International journal of STD \& AIDS 2020:956462420905275. doi: 10.1177/0956462420905275.

3. Van Bergen J, Dekker J, Boeke J, et al. NHG-Standard Het soa-consult (eerste herziening). HUISARTS EN WETENSCHAP-NEDERLANDS- 2013;56(9):450-63.

4. Barbee LA, Golden MR. Editorial Commentary: When to Perform a Test of Cure for Gonorrhea: Controversies and Evolving Data. Clin Infect Dis 2016;62(11):1356-9. doi: 10.1093/cid/ciw142 [published Online First: 2016/03/11].

5. Lazaro N. Sexually Transmitted Infections in Primary Care 2013 (RCGP/BASHH) available at www. rcgp.org and www.bashh.org/guidelines [cited 201720 April]. accessed 4-11-2019.

6. Bignell C, Unemo M, European STIGEB. 2012 European guideline on the diagnosis and treatment of gonorrhoea in adults. International journal of STD \& AIDS 2013;24(2):85-92. doi: $10.1177 / 0956462412472837$.

7. Workowski KA. Centers for Disease Control and Prevention Sexually Transmitted Diseases Treatment Guidelines. Clin Infect Dis 2015;61 Suppl 8:S759-62. doi: 10.1093/cid/civ771.

8. World Health Organization Department of Reproductive Health and Research. Global action plan to control the spread and impact of antimicrobial resistance in Neisseria gonorrhoeae 2012 [Available from: https://www.who.int/reproductivehealth/publications/rtis/9789241503501/en/.

9. den Heijer CDJ, Hoebe C, van Liere G, et al. A comprehensive overview of urogenital, anorectal and oropharyngeal Neisseria gonorrhoeae testing and diagnoses among different STI care providers: a cross-sectional study. BMC Infect Dis 2017;17(1):290. doi: 10.1186/s12879-017-2402-0.

10. Hosenfeld CB, Workowski KA, Berman S, et al. Repeat infection with Chlamydia and gonorrhea among females: a systematic review of the literature. Sex Transm Dis 2009;36(8):478-89. doi: 10.1097/OLQ.0b013e3181a2a933.

11. Fung M, Scott KC, Kent CK, et al. Chlamydial and gonococcal reinfection among men: a systematic review of data to evaluate the need for retesting. Sex Transm Infect 2007;83(4):304-9. doi: 10.1136/ sti.2006.024059.

12. Nwokolo NC, Dragovic B, Patel S, et al. 2015 UK national guideline for the management of infection with Chlamydia trachomatis. International journal of STD \& AIDS 2016;27(4):251-67. doi: 10.1177/0956462415615443.

13. Kampman C, Koedijk F, Driessen-Hulshof H, et al. Retesting young STI clinic visitors with urogenital Chlamydia trachomatis infection in the Netherlands; response to a text message reminder and reinfection rates: a prospective study with historical controls. Sex Transm Infect 2016;92(2):124-9. doi: 10.1136/sextrans-2015-052115. 
14. Dukers-Muijrers NH, Theunissen KA, Wolffs PT, et al. Acceptance of Home-Based Chlamydia Genital and Anorectal Testing Using Short Message Service (SMS) in Previously Tested Young People and Their Social and Sexual Networks. PLoS One 2015;10(7):e0133575. doi: 10.1371/journal. pone.0133575.

15. Yeung A, Temple-Smith M, Spark S, et al. Improving chlamydia knowledge should lead to increased chlamydia testing among Australian general practitioners: a cross-sectional study of chlamydia testing uptake in general practice. BMC Infect Dis 2014;14:584. doi: 10.1186/s12879-014-0584-2.

16. Rose SB, Garrett SM, Hutchings D, et al. Clinician education, advice and SMS/text reminders improve test of reinfection rates following diagnosis of Chlamydia trachomatis or Neisseria gonorrhoeae: before and after study in primary care. BMJ Sex Reprod Health 2019 doi: 10.1136/ bmjsrh-2018-20018.5

17. den Heijer CD, van Liere GA, Hoebe CJ, et al. Who tests whom? A comprehensive overview of Chlamydia trachomatis test practices in a Dutch region among different STI care providers for urogenital, anorectal and oropharyngeal sites in young people: a cross-sectional study. Sex Transm Infect 2016;92(3):211-7. doi: 10.1136/sextrans-2015-052065.

18. Chan PA, Robinette A, Montgomery M, et al. Extragenital Infections Caused by Chlamydia trachomatis and Neisseria gonorrhoeae: A Review of the Literature. Infect Dis Obstet Gynecol 2016;2016:5758387. doi: 10.1155/2016/5758387.

19. Trienekens SC, van den Broek IV, Donker GA, et al. Consultations for sexually transmitted infections in the general practice in the Netherlands: an opportunity to improve STI/HIV testing. BMJ Open 2013;3(12):e003687. doi: 10.1136/bmjopen-2013-003687.

20. De Vries H, van Dam A, Bax C. Seksueel Overdraagbare Aandoeningen Multidisciplinaire Richtlijn Update 20182018 [Available from: www.soaaids.nl/nl/professionals/beroepsgroep/arts/ professioneel-handelen/de-specialist accessed 3-9-2018.

21. Hsu KK, Molotnikov LE, Roosevelt KA, et al. Characteristics of Cases With Repeated Sexually Transmitted Infections, Massachusetts, 2014-2016. Clin Infect Dis 2018;67(1):99-104. doi: 10.1093/ cid/ciy029.

22. Dirks JA, van Liere GA, Bogers S, et al. Natural Course of Chlamydia trachomatis Bacterial Load in the Time Interval between Screening and Treatment in Anogenital Samples. PLoS One 2015;10(12):e0145693. doi: 10.1371/journal.pone.0145693.

23. Dukers-Muijrers N, Wolffs PFG, Vries $\mathrm{H}$, et al. Treatment effectiveness of azithromycin and doxycycline in uncomplicated rectal and vaginal Chlamydia trachomatis infections in women: a multicentre observational study (FemCure). Clin Infect Dis 2019 doi: 10.1093/cid/ciz050.

24. Dukers-Muijrers NH, Wolffs PF, Eppings L, et al. Design of the FemCure study: prospective multicentre study on the transmission of genital and extra-genital Chlamydia trachomatis infections in women receiving routine care. BMC Infect Dis 2016;16:381. doi: 10.1186/s12879-016-1721-x.

25. Dirks JAMC, Hoebe CJPA, van Liere GAFS, et al. Standardisation is necessary in urogenital and extragenital <em>Chlamydia trachomatis</em> bacterial load determination by quantitative PCR: a review of literature and retrospective study. Sexually Transmitted Infections 2019:sextrans-2018-053522. doi: 10.1136/sextrans-2018-053522. 
26. van Rooijen MS, van der Loeff MF, Morre SA, et al. Spontaneous pharyngeal Chlamydia trachomatis RNA clearance. A cross-sectional study followed by a cohort study of untreated STI clinic patients in Amsterdam, The Netherlands. Sex Transm Infect 2015;91(3):157-64. doi: 10.1136/ sextrans-2014-051633.

27. Haggerty CL, Gottlieb SL, Taylor BD, et al. Risk of sequelae after Chlamydia trachomatis genital infection in women. J Infect Dis 2010;201 Suppl 2:S134-55. doi: 10.1086/652395.

28. Gupta K, Bakshi RK, Van Der Pol B, et al. Repeated Chlamydia trachomatis infections are associated with lower bacterial loads. Epidemiol Infect 2018:1-3. doi: 10.1017/S0950268818002704.

29. Walker J, Tabrizi SN, Fairley CK, et al. Chlamydia trachomatis incidence and re-infection among young women--behavioural and microbiological characteristics. PLoS One 2012;7(5):e37778. doi: 10.1371/journal.pone.0037778.

30. Kong FY, Tabrizi SN, Fairley CK, et al. The efficacy of azithromycin and doxycycline for the treatment of rectal chlamydia infection: a systematic review and meta-analysis. The Journal of antimicrobial chemotherapy 2015;70(5):1290-7. doi: 10.1093/jac/dku574 [published Online First: 2015/02/01].

31. Kong FY, Tabrizi SN, Law M, et al. Azithromycin versus doxycycline for the treatment of genital chlamydia infection: a meta-analysis of randomized controlled trials. Clin Infect Dis 2014;59(2):193205. doi: 10.1093/cid/ciu220.

32. Janssen KJ, Hoebe CJ, Dukers-Muijrers NH, et al. Viability-PCR Shows That NAAT Detects a High Proportion of DNA from Non-Viable Chlamydia trachomatis. PLoS One 2016;11(11):e0165920. doi: 10.1371/journal.pone.0165920.

33. Janssen KJH, Wolffs P, Lucchesi M, et al. Assessment of rectal Chlamydia trachomatis viable load in women by viability-PCR. Sex Transm Infect 2020;96(2):85-88. doi: 10.1136/sextrans-2019-054002. 



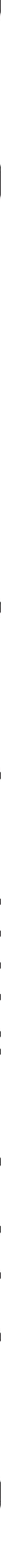




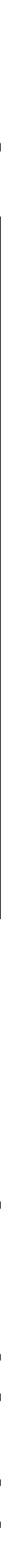




\section{Summary}

The studies included in this thesis provide insight in test practices of general practitioners (GPS), characteristics of patients repeatedly infected with Chlamydia trachomatis (CT) or Neisseria gonorrhoeae (NG), the CT bacterial load, and geographical analyses to inform CT and NG control.

In chapter 2, CT and NG test of cure (TOC), and retesting rates of 48 Dutch GP practices were analyzed in the South-Western part of Limburg in the Netherlands between January 2011 and July 2016. Relatively high CT (20\%) and NG (25\%) TOC rates were observed. Whereas relatively low CT (24\%) and NG (15\%) retesting rates were observed. Notably, in only $0.3 \%$ of the CT and NG consultations patients were tested at extragenital sites. Due to the relatively high TOC rates, overtreatment could have occurred. Moreover, due to the relatively low retesting and extragenital testing rates (repeat) infections were likely missed leading to ongoing transmission of CT and NG.

In chapter 3, we assessed whether patients repeatedly infected with CT were different to patients repeatedly tested negative for CT. In this study, 17,616 patients tested for CT in a sexually transmitted infections (STI) clinic, at a GP practice or in a hospital were included in the period between January 2011 and July 2018. Younger patients (aged <25 years) and patients co-infected with HIV or NG had more often repeat CT infections. These patients accounted for $20 \%$ of all diagnosed CT infections and are likely at highest risk for transmitting and acquiring CT. Notably, $41 \%$ of the CT patients were not retested after CT diagnosis and therefore lost to care, indicating missed repeat infections and potentially ongoing transmission of CT.

Similar to chapter 3, but than for NG, we assessed in chapter 4 whether patients from our STI clinic, GPS or hospitals repeatedly infected with NG were different compared to patients repeatedly tested negative for NG. Patients $(n=16,662)$ were tested for NG in the period January 2011 and July 2018. $0.2 \%$ of the patients ( $n=40)$ with repeat NG infections accounted for $23 \%$ of all diagnosed NG infections. Patients with repeat NG infections were almost all MSM and visiting the STI clinic. Notably, one out of three NG patients were not retested after NG diagnosis. Therefore, repeat NG infections were likely missed potentially leading to ongoing transmission of NG. It remains important to test and retest for NG, especially in MSM, in order to halt transmission.

In chapter 5, we assessed whether patients visiting different STI care providers (STI clinic, GPs, or the hospital) have a different urogenital CT bacterial load. In this study, CT positive urogenital samples of men and women were included between 2012 and 2016 (n=3,670). Men visiting different STI care providers had a similar urogenital CT load and likely similar chances of transmission and sequelae. Women visiting the GP had a higher CT load compared to women visiting the STI clinic, although the difference was small. Notably, women concurrently infected with urogenital and anorectal CT, and women who were not anorectally tested had a substantial higher urogenital CT load compared to women with no anorectal 
infections. Likely those women who are concurrently infected with urogenital and (untested) anorectal CT have higher chances of transmission and sequelae.

In chapter 6, we assessed the CT bacterial load of different urogenital and extragenital sample types, and therefore different anatomic locations. In this largest study to date, all CT positive urogenital and extragenital samples of men and women were included between 2012 and 2018 ( $n=7,900)$. Vaginal swabs had the highest CT load, followed by urine samples, anorectal swabs, and oropharyngeal swabs. The most notable finding was that men and women have an equal extragenital load and therefore likely similar clinical relevance despite different testing guidelines for men and women. Furthermore, older patients and patients co-infected with HIV had a lower urogenital CT load, suggesting exposure to previous CT infections potentially leading to partially immunity reducing CT load.

In chapter 7, we assessed the urogenital and extragenital CT bacterial load of men ( $n=221)$ and women ( $n=208$ ) repeatedly infected with CT between 2012 and 2018. Men and women repeatedly infected with CT have a lower urogenital CT load. Such association was not observed for anorectal infections of men and women. Therefore, partial immunity could play a role in repeat urogenital infections, but likely not in repeat anorectal infections.

In chapter 8, epidemiological analyses were compared with geographical cluster analyses for detecting high risk CT and NG geographical areas. For this study we included CT and NG consultations of all STI clinics in the Netherlands between 2013 and 2015 ( $n=379,926$ ). Low SES- and urban areas were associated with high CT and NG detections rates. The geographical clusters were mainly observed in areas with high population densities, thus urban areas. In one STI clinic region, the geographical cluster analyses did not visualize any NG cluster, whereas 489 NG cases were diagnosed in that region. The number needed to test for CT and NG was lower for the geographical cluster analyses (range: 57-369) compared to the epidemiological analyses (range: 93-2,104) per STI clinic region. This could indicate the efficiency of geographical analyses for targeting STI control outreach activities. However, a combination of both approaches is imperative in providing geographical insight in CT testing and positivity and to avoid missed cases.

In chapter 9, all chapters were discussed in a general discussion with concluding remarks and future directions for research. 


\section{Nederlandse samenvatting}

De studies in dit proefschrift geven inzicht in test praktijken van huisartsen, sociaal demografische karakteristieken van patiënten met herhaalde Chlamydia trachomatis (CT) of Neisseria gonorrhoeae (NG) infecties, de CT bacteriële load en geografische analysen voor het informeren van CT- en NG bestrijding.

In hoofdstuk 2, werden CT- en NG test-of-cure en hertest percentages van 48 huisartspraktijken geanalyseerd in het Zuidwesten van Limburg tussen januari 2011 en juli 2016. We vonden relatieve hoge CT (20\%) en NG (25\%) test-of-cure percentages. Daarentegen werden relatieve lage CT (24\%) en NG (15\%) hertest percentages gemeten. Een opmerkelijke bevinding was dat in slechts $0,3 \%$ van de CT- en NG consultaties patiënten extra-genitaal (oraal of anaal) getest werden. Overbehandeling van CT en NG heeft mogelijk plaats gevonden doordat relatieve hoge test-of-cure percentages werden waargenomen. Daarnaast zijn waarschijnlijk herinfecties en extra-genitale infecties gemist door het lage percentage hertesten en extra-genitale testen. Dit kan leiden tot verdere verspreiding van CT en NG.

In hoofdstuk $\mathbf{3}$ hebben we sociaal demografische kenmerken van patiënten met herhaalde CT-infecties vergeleken met personen die frequent negatief testen voor CT. In deze studie hebben we 17.616 personen, die op CT getest waren door de GGD, huisartsen of medisch specialisten, geïncludeerd tussen januari 2011 en juli 2018. Patiënten jonger dan 25 jaar en patiënten met een HIV of NG co-infectie hadden significant vaker een herhaalde CT-infectie. Deze patiënten met herhaalde CT-infecties zorgden voor $20 \%$ van de gediagnosticeerde CT-infecties en lopen waarschijnlijk het grootste risico om CT te verspreiden in de populatie. Opmerkelijk was dat 41\% van de CT-patiënten niet werden gehertest na diagnose en uit de zorg verdwenen. Dit leidt mogelijk tot gemiste CT-infecties en verdere verspreiding van CT.

Vergelijkbaar als in hoofdstuk 3, hebben we in hoofdstuk 4 patiënten met herhaalde NG-infecties vergeleken met personen die frequent negatief testen voor NG. Alle personen die getest werden op NG ( $\mathrm{n}=16.662)$ door de GGD, huisartsen en medisch specialisten, werden geïncludeerd in de periode tussen januari 2011 en juli 2018. Slechts $0.2 \%$ ( $n=40)$ van de geteste patiënten, met herhaalde NG-infecties, zorgden voor $23 \%$ van alle gediagnosticeerde NG-infecties. Patiënten met herhaalde NG-infecties waren voornamelijk mannen die seks hebben met mannen (MSM) en gediagnosticeerd door de GGD. Opmerkelijk was dat 1 op de 3 patiënten met een NG infectie niet werden gehertest na diagnose. Daardoor zijn waarschijnlijk herhaalde NG-infecties gemist wat kan leiden tot verdere verspreiding van NG. Het is, en blijt, belangrijk om risicogroepen zoals MSM te (her)testen om zo verdere NG-verspreiding tegen te gaan.

In hoofdstuk 5 hebben we de urogenitale CT bacteriële load van verschillende populaties (GGD-populatie, huisarts populatie, medisch specialisten populatie) vergeleken. In deze studie hebben we urogenitale CT positieve samples van mannen en vrouwen geïncludeerd in de periode tussen 2012 en 2016 (n=3.670). Mannen van verschillende populaties hadden dezelfde urogenitale CT bacteriële load en waarschijnlijk vergelijkbare kansen op verdere 
verspreiding. Daarentegen hadden vrouwen die de huisarts bezochten een hogere urogenitale CT bacteriële load dan vrouwen die de GGD bezochten, echter was het verschil klein. Opmerkelijk was dat vrouwen met een gelijktijdige urogenitale en anale CT-infectie een aanzienlijk hogere urogenitale CT bacteriële load hadden in vergelijking met vrouwen die geen anale infectie hadden. Waarschijnlijk hebben vrouwen met een gelijktijdige urogenitale en anorectale chlamydia-infectie een hogere kans op complicaties en verspreiding van CT.

In hoofdstuk $\mathbf{6}$ hebben we de CT bacteriële load van verschillende lichaamslocaties (urogenitaal, anaal en oraal) vergeleken en getoetst. In deze grootste studie tot nu toe werden alle CT positieve urogenitale en extra-genitale samples van mannen en vrouwen in de periode 2012 en 2018 (n=7.900) geïncludeerd. De meest opmerkelijke bevinding was dat mannen en vrouwen een vergelijkbare anale en orale CT bacteriële load hadden. Daardoor hebben extra-genitale infecties van mannen en vrouwen waarschijnlijk een vergelijkbare impact op transmissie ondanks verschillende test richtlijnen voor mannen en vrouwen. Daarnaast hadden oudere patiënten (>25 jaar) en patiënten met een HIV co-infectie een lagere urogenitale CT bacteriële load mogelijk door gedeeltelijke immunisatie als gevolg van herinfecties.

In hoofdstuk 7 hebben we de urogenitale en extra-genitale CT bacteriële load van mannen $(n=221)$ en vrouwen $(n=208)$ met herhaalde CT-infecties vergeleken in de periode tussen 2012 en 2018. Mannen en vrouwen met herhaalde CT-infecties hebben een lagere urogenitale CT bacteriële load in vergelijking met een eerdere urogenitale CT-infectie. Voor herhaalde anale infecties van mannen en vrouwen was geen verschil in de CT bacteriële load waarneembaar. Gedeeltelijke immunisatie kan een rol spelen in herhaalde urogenitale CT-infecties, waarschijnlijk niet in herhaalde anale CT-infecties.

In hoofdstuk 8 hebben we een epidemiologisch analyse vergeleken met een geografische cluster analyse voor het detecteren van hoog risicogebieden voor CT en NG. In deze studie hebben we alle CT en NG testen van GGD'en in Nederland tussen 2013 en 2015 geïncludeerd ( $n=379.926)$. Lage sociaaleconomische status- en stedelijke gebieden waren geassocieerd met hoge CT- en NG vindpercentages. Daarnaast werden de geografische clusters voornamelijk waargenomen in gebieden met hoge bevolkingsdichtheden. Dit waren dus voornamelijk de steden. In één GGD-regio werden geen geografische NG-clusters waargenomen, terwijl 489 NG-cases waren gediagnosticeerd in diezelfde GGD-regio. Het aantal personen dat getest zou moeten worden om één CT- of NG infectie te diagnosticeren was lager voor de geografische cluster analyse (range: 57-369) in vergelijking met de epidemiologisch analyse (range: 93-2.104) per GGD-regio. Dit zou kunnen duiden op de efficiëntie van geografische analysen voor potentiële outreach activiteiten. Echter, een combinatie van een geografische- en epidemiologisch analyse is noodzakelijk om een regio in kaart te brengen en om gemiste infecties te voorkomen.

In hoofdstuk 9 werden alle voorgaande hoofdstukken besproken in een algemene discussie inclusief afsluitende opmerkingen en aanbevelingen voor verder onderzoek. 


\section{Valorization}

Besides scientific relevance, this thesis also has implications for society and sexual health care test practices. Our results provided insight in Chlamydia trachomatis (CT) and Neisseria gonorrhoeae (NG) test practices, the CT bacterial load and geographical mapping and analyses. The practical and societal implications of these results, e.g. on testing and treatment practices, are mainly indirect. The results of this thesis could fuel discussions for testing and treatment guidelines, serve as a basis for new research, and could be used to inform public health efforts.

\section{Test of cure, retesting and extragenital testing}

Next to sexually transmitted infection (STI) clinics, general practitioners (GPS) are one of the main STI care providers. Optimizing testing strategies for GPs could have huge impact on CT and NG control.

Relatively high unnecessary CT and NG test of cure (TOC) rates were observed among patients visiting the GP. However, according to (inter)national guidelines for all care professionals, GPs are recommended against a CT or NG TOC; only in specific indicated cases, as described in the Dutch national guideline for GPS.

Re-testing, i.e. testing after 3 months to 12 months to detect a possible re-infection, is essential to trace hidden infections. During a consultation, the GP could check when the last CT or NG test was performed and should advice the patient to retest after three months. This can also be done by a trained GP assistant on the phone prior to a consultation. This will lead to less unnecessary STI consultations and costs. Moreover, the combination of text messages, clinician education and advice have shown to increase CT and NG retesting rates in primary care in New Zealand. Furthermore, sending a home sampling kit for self-testing six months after diagnosis could increase retesting rates and detect potential hidden CT and NG infections. The latter is currently assessed in the "Chlamydia: Hidden Infectious Management in Primary care" (CHIMP) study which was informed by results from the studies in this thesis.

The Dutch national guideline for GPs (2013) will be updated, since in the last decade many new insights in CT and NG control have been published. For example, the Dutch GP guideline only recommend CT positive patients to retest within one year. However, NG positive patients, maybe even more important due to increasing antimicrobial resistance of NG, should also always be retested within 3 to 12 months. Many studies show high (up to 40\%) reinfection rates of $N G$ within one year.

According to the Dutch GP guideline, the first-choice treatment for CT is azithromycin. As extragenital testing was rarely performed by GPs (in $0.3 \%$ of the CT test consultations), likely all CT positive patients were treated with first choice treatment azithromycin. As a consequence, potential undiagnosed anorectal infections were likely not adequately treated, 
possibly leading to ongoing transmission within the population. Policymakers and GPs may consider doxycycline as first line treatment instead of azithromycin as (1) many studies showing that concurrent urogenital and anorectal infections are highly common among women, (2) the effectiveness of doxycycline is high and exceeds that of azithromycin for urogenital and anorectal CT in women, and (3) extragenital testing was rarely performed in GP settings. Universal doxycycline use could lead to treatment of potential undiagnosed anorectal CT infections, likely reduce ongoing transmission for the benefit of public health. Still, treatment adherence of doxycycline could be an issue and should be investigated further in different populations and settings.

Also based on the results of our study in the GP setting, the currently ongoing "Chlamydia: Hidden Infectious Management in Primary care" (CHIMP) has been set up to increase extragenital CT (re)testing rates in the GP setting. Furthermore, several spin-off studies were initiated, such as "FemCure" and "CHLAMOUR", to study the burden, transmission, and cure of CT at different anatomical locations including anorectal CT.

Lastly, the Dutch National Journal of Medicine ("Het Nederlands Tijdschrift voor Geneeskunde") published an article based on our study among GP patients entitled "Follow-up of CT and NG can be better" ("Nacontrole bij chlamydia en gonorroe kan beter"). The journal is specific for Dutch medical doctors and the article in the special issue related to sexual health could have impact in the awareness of TOC, retesting and extragenital testing for CT and NG in the GP setting.

\section{Chlamydia trachomatis bacterial load}

In addition to our finding that extragenital testing is rarely performed by GPs, our findings concerning the CT bacterial load could fuel discussion about the need for extragenital CT testing and the appropriate treatment. According to STI testing guidelines, men who have sex with men (MSM) should always be tested anorectally, and on indication (oral sex or symptoms) orally. In contrast, women are only tested on extragenital anatomic locations based on indication (anorectal or oropharyngeal sex, and symptoms). We showed that women concurrently infected with urogenital and anorectal, and women who were not anorectally tested for CT, had a substantial higher urogenital CT bacterial load compared to women who were anorectally CT negative. Moreover, despite differences in testing guidelines for MSM and women, we observed an equal extragenital CT bacterial load of men and women. These findings could indicate that anorectal CT infections in women are "real" infections regardless whether these result from potential autoinoculation from the urogenital site or not. Therefore, the findings in this thesis may add evidence to the potential transition from azithromycin to doxycycline as first choice treatment for CT in the future. Policymakers should consider all the existing literature concerning anorectal CT infections in men and women when updating STI testing and treatment guidelines. 


\section{Geographical mapping and analyses}

In two studies of this thesis we used geographical mapping to identify high risk CT and NG areas. In chapter 4, local geographical areas of patients repeatedly infected with NG were detected. Geographical maps are attractive to read and can be very informative (although also sensitive to misinterpretation or visual bias). Furthermore, geographical maps can be used as a basis for potential outreach activities. For example, a relatively high number of patients, who tested NG positive and who were not retested, were living close to the STI clinic in Maastricht. As people with the same sociodemographic characteristics tend to cluster geographically, likely more undiagnosed NG patients are living in this area. Without geographical mapping such valuable information was not known. Active testing in this area could detect potential hidden NG cases. Another question is whether those NG patients living nearby the STI clinic were not retested, since patients living nearby STI clinic have easier access to the STI clinic than, for example, patients living outside the city. However, caution is needed when interpreting geographical maps including data of the tested population. The hidden population at risk, those who never test for CT or NG, are not visualized and could also have high impact on transmission. Maybe even more in non-urban areas compared to urban areas since access to STI clinics could be a barrier. Likely unawareness of the importance of retesting may also play a role. Furthermore, we included maps of the GP population who had repeat NG infections and who were not retested after diagnoses. These unique geographical maps can be used to inform GPs in high risk NG areas. This could increase the awareness and importance of NG (repeat) testing among GPS, since we noticed that not all GPs test simultaneously for $\mathrm{CT}$ and $\mathrm{NG}$ at the same consultation.

In our second study, we geographically mapped and analyzed all CT and NG consultations of all STI clinics in the Netherlands. The geographical cluster analyses showed various CT and NG clusters throughout the country. As the Netherlands is divided in eight STI clinic regions, the results are communicated to every STI clinic region.

Moreover, the study is communicated to the National Institute for Public Health and the Environment (in Dutch: RIVM). However, geographical analyses including a large area (such as a whole country) is less informative and difficult for practical use since relatively large geographical clusters were produced. Therefore, an additional geographical analyses per STI clinic region should be performed to obtain more detailed geographical clusters. For example, the National Institute for Public Health and the Environment could perform geographical analyses per STI clinic region and could add the results to the yearly booklet "Sexually transmitted infections in the Netherlands". The results per STI clinic region could be used for targeted testing and specific testing guidelines per STI clinic region. The geographical analyses could be performed, evaluated and adjusted every year for optimizing CT and NG control. However, expert knowledge is needed to perform and interpret geographical analyses. Furthermore, geographical cluster analyses can only be performed with a sufficient number of cases to obtain reliable results, which can be a problem in smaller geographical areas with 
low number of cases. Lastly, and very important, our geographical analyses included only national STI clinic data. Therefore, approximately half of the CT/NG tests and infections were visualized since GP data were not used. Geographical analyses including GP data would lead to different results and therefore different geographical clusters. However, a geographical cluster analyses using GP data could still be very useful to inform local CT and NG control where ideally public health services and GPs work together. 


\section{Dankwoord}

Na vier jaar lang aan dit proefschrift te hebben gewerkt, is het dan eindelijk klaar. Ik heb veel geleerd over chlamydia, gonorroe en epidemiologie. Daarom wil ik iedereen, die op welke wijze dan ook heeft bijgedragen aan dit proefschrift, hartelijk bedanken! 


\section{List of publications}

- Wijers J, van Liere G, Hoebe C, et al. Test of cure, retesting and extragenital testing practices for Chlamydia trachomatis and Neisseria gonorrhoeae among general practitioners in different socioeconomic status areas: A retrospective cohort study, 2011-2016. PLoS One 2018;13(3):e0194351. doi: 10.1371/journal.pone.0194351

- Wijers J, Hoebe C, van Liere G, et al. Chlamydia trachomatis bacterial load, estimated by Cq values, in urogenital samples from men and women visiting the general practice, hospital or STI clinic. PLoS One 2019;14(4):e0215606. doi: 10.1371/journal.pone.0215606

- Wijers J, Dukers-Muijrers N, van Liere G, et al. Men and women have an equal oropharyngeal and anorectal Chlamydia trachomatis bacterial load: a comparison of three anatomic sites. J Infect Dis 2019 doi: 10.1093/infdis/jiz668

- Wijers J, Hoebe C, Dukers-Muijrers N, et al. The Characteristics of Patients Frequently Tested and Repeatedly Infected with Neisseria gonorrhoeae. Int J Environ Res Public Health 2020;17(5) doi: 10.3390/ijerph17051495

- Dukers-Muijrers N, Heijman T, Gotz HM, Zaandam P, Wijers J, et al. Participation, retention, and associated factors of women in a prospective multicenter study on Chlamydia trachomatis infections (FemCure). PLoS One 2020;15(3):e0230413. doi: 10.1371/journal.pone.0230413

- Wijers J, van Liere G, Dukers-Muijrers N, et al. Men and women repeatedly infected with Chlamydia trachomatis have a lower urogenital bacterial load. Sex Transm Dis 2020 doi: 10.1097/ OLQ.0000000000001219

- Wijers J, Dukers-Muijrers N, Hoebe C, et al. The characteristics of patients frequently tested and repeatedly infected with Chlamydia trachomatis in Southwest Limburg, the Netherlands. BMC Public Health 2020;20(1):1239. doi: 10.1186/s12889-020-09334-9 
